\title{
Darstellung neuer Organometallhalogenide mit Elementen der 13. und 14. Gruppe des Periodensystems und Versuche zur Synthese von Organogermanolen
}

\author{
Dissertation \\ zur Erlangung des Doktorgrades \\ der Mathematisch-Naturwissenschaftlichen Fakultäten \\ der Georg-August-Universität zu Göttingen
}

vorgelegt von

Holger Hohmeister

aus Eschwege

Göttingen 2003 
D 7

Referent:

Prof. Dr. Dr. h. c. mult. H. W. Roesky

Koreferent:

Prof. Dr. F. Meyer

Tag der mündlichen Prüfung: 2. Juli 2003 
Für meine Frau Birte, mein Baby, meine Eltern und Frenzy (15.8.1999 - 4.4.2003) 
Die vorliegende Arbeit wurde im Arbeitskreis von Herrn Prof. Dr. Dr. h. c. mult. H. W. Roesky im Institut für Anorganische Chemie der Georg-August-Universität Göttingen in der Zeit von Juni 1998 bis März 2003 angefertigt.

Meinem verehrten Lehrer,

\section{Herrn Prof. Dr. Dr. h. c. mult. H. W. Roesky}

danke ich sehr herzlich für die interessante Themenstellung, die ausgezeichneten Arbeitsbedingungen, sein stetes Interesse am Fortgang dieser Arbeit sowie die großzügige Unterstützung.

Für die Ausführung der spektroskopischen und analytischen Arbeiten danke ich den Herren Dr. D. Böhler (MS), T. Schuchardt (MS), Dr. G. Elter (NMR), R. Schöne (NMR), W. Zolke (NMR), H.-J. Feine (IR), M. Hesse (IR) und den Damen A. Rehbein (MS), H. Rusev (Analysen), S. Petrich (Analysen) und A. Wraage (Analysen).

Mein besonderer Dank gebührt den Herren Prof. Dr. J. Magull, Dr. M. Noltemeyer und H.-G. Schmidt für die Durchführung der EinkristallRöntgenstrukturanalysen.

Des weiteren danke ich Herrn Dr. M. Witt für die ständige Hilfsbereitschaft und die anregenden wissenschaftlichen Diskussionen und die Korrektur dieser Arbeit.

Meinen Kollegen im Arbeitskreis, insbesondere den Herren Dr. D. H. Hamilton, Dr. H. Wessel, Dr. C. Rennekamp, Dr. J. Prust, Dr. S. D. Waezsada, Dr. W. Zheng, Dr. C. Manthey, J. Janssen, P. Lobinger, A. Stasch, T. Blunck und H. Zhu möchte ich für das sehr angenehme Arbeitsklima und die ständige Hilfsbereitschaft danken. Besonders danke ich meiner Frau B. Hohmeister sowie meinen Eltern für ihre Unterstützung. Ebenso danke ich allen Ungenannten, die zum Fortschreiten dieser Arbeit beigetragen haben. 


\section{Inhaltsverzeichnis}

Inhaltsverzeichnis $\quad$ I

Abkürzungsverzeichnis $\quad$ VI

1. Einleitung 1

1.1. Organoaluminiumfluoride 1

1.2. Terphenyle und Vinamidine als Ligandensysteme 3

1.3. Organasilanole und -germanole 5

1.4. Sesquichalcogenide 5

$\begin{array}{llr}\text { 2. } & \text { Aufgabenstellung } & 7\end{array}$

$\begin{array}{llr}\text { 3. Theoretischer Teil } & 8\end{array}$

3.1. Fluorierung von Alumazen $\left[\mathrm{MeAIN}\left(2,6-i-\mathrm{Pr}_{2} \mathrm{C}_{6} \mathrm{H}_{3}\right)\right]_{3} \quad(1)$ mit 8 $\mathrm{Me}_{3} \mathrm{SnF}$

3.1.1. Darstellung von $\left[\mathrm{FAlN}\left(2,6-i-\mathrm{Pr}_{2} \mathrm{C}_{6} \mathrm{H}_{3}\right)\right]_{3} \cdot 3$ THF (7) und $\left[\mathrm{Me}_{2} \mathrm{SnN}(2,6-\quad 10\right.$ $\left.\left.i-\mathrm{Pr}_{2} \mathrm{C}_{6} \mathrm{H}_{3}\right)\right]_{2}(\mathbf{8})$

3.1.2. Diskussion der Einkristall-Röntgenstrukturanalyse von 7

3.1.3. Diskussion der Einkristall-Röntgenstrukturanalyse von 8

3.2. Versuch zur Umsetzung von $\mathbf{1}$ mit $\boldsymbol{n}-\mathrm{Bu}_{\mathbf{4}} \mathbf{N}\left[\mathrm{HF}_{2}\right]$

3.3. Umsetzung von 1 mit Borhalogeniden und Boranen 16

3.3.1. Umsetzung von $\mathbf{1}$ mit F$_{3} \mathrm{~B} \cdot \mathrm{OEt}_{2}$

3.3.2. Umsetzung von $\mathbf{1}$ mit $\mathrm{Cl}_{3} \mathrm{~B} ;$ Darstellung von $[\mathrm{ClAlN}(2,6-i-\quad 17$ $\left.\left.\mathrm{Pr}_{2} \mathrm{C}_{6} \mathrm{H}_{3}\right)\right]_{3} \cdot 3 \mathrm{THF}(\mathbf{9})$

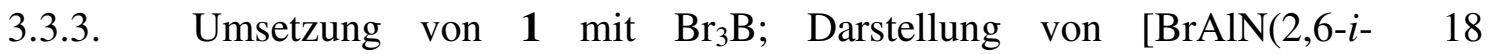
$\left.\left.\operatorname{Pr}_{2} \mathrm{C}_{6} \mathrm{H}_{3}\right)\right]_{3} \cdot 3$ THF (10) 
3.3.4. Versuch zur Umsetzung von $\mathbf{1}$ mit $\mathrm{I}_{3} \mathrm{~B} \quad 19$

3.3.5. Reaktion von 1 mit $\mathrm{H}_{3} \mathrm{~B} \cdot \mathrm{THF} ;$ Darstellung von $[\operatorname{MeAlN}(2,6-i-\quad 19$ $\left.\left.\mathrm{Pr}_{2} \mathrm{C}_{6} \mathrm{H}_{3}\right)\right]_{3} \cdot 3$ THF (11)

3.4. Versuch zur Umsetzung von $1 \mathrm{mit} \mathbf{P h S i}(\mathrm{C} \equiv \mathrm{CH})_{3} \quad 20$

3.5. Darstellung und Hydrolyse von monoorganylsubstituierten 21 Germaniumtrihalogeniden

3.5.1. Darstellung von 2,6-Bis(2`,6`-dimethylphenyl)-1-iodbenzol (12) 21

3.5.2. Darstellung und Struktur von 2,6-Bis(2‘,6'-dimethylphenyl)- 24 phenylgermaniumtrichlorid $\left(\mathrm{R}^{*} \mathrm{GeCl}_{3}\right)(\mathbf{1 3})$

3.5.3. Darstellung und Struktur von 2,6-Bis(2‘,6`-dimethylphenyl)- 26 phenylgermaniumtribromid $\left(\mathrm{R}^{*} \mathrm{GeBr}_{3}\right)(\mathbf{1 4})$

3.5.4. Darstellung von 2,6-Bis(2‘,6`-dimethylphenyl)phenylgermanium- 27 triiodid (15)

3.5.5. Darstellung von 2,6-Bis(2‘,6`-dimethylphenyl)phenylgermanium- 28 trifluorid (16)

3.5.6. Darstellung von $\operatorname{DippN}\left(\mathrm{SiMe}_{3}\right) \mathrm{GeF}_{3}(\mathbf{1 7}) \quad 29$

3.6. Versuche zur Hydrolyse von Monoorganylgermanium- 29 trihalogeniden

3.6.1. Umsetzung von $\operatorname{DippN}\left(\mathrm{SiMe}_{3}\right) \mathrm{GeBr}_{3}$ mit $\mathrm{PhNH}_{2}$ und $\mathrm{H}_{2} \mathrm{O} \quad 30$

3.6.2. Umsetzung von $\operatorname{DippN}\left(\mathrm{SiMe}_{3}\right) \mathrm{GeBr}_{3}$ mit Pyridin und $\mathrm{H}_{2} \mathrm{O} ; \quad 30$ Darstellung von $\left[\left(\mathrm{DippN}\left(\mathrm{SiMe}_{3}\right) \mathrm{Ge}\right)_{4} \mathrm{O}_{6}\right](\mathbf{1 8})$

3.6.3. Umsetzung von $\operatorname{DippN}\left(\mathrm{SiMe}_{3}\right) \mathrm{GeBr}_{3}$ mit $\mathrm{H}_{2} \mathrm{O}_{2}$

3.6.4. Umsetzung von (DippN $\left.\left(\mathrm{SiMe}_{3}\right) \mathrm{Ge}\left(\mathrm{NH}_{2}\right)_{2}\right)_{2} \mathrm{NH}$ und $\mathrm{H}_{2} \mathrm{O}$

3.6.5. Umsetzung von $\operatorname{DippN}\left(\mathrm{SiMe}_{3}\right) \mathrm{GeBr}_{3}$ mit $\mathrm{KOH}$ im Zwei- 32 
Phasensystem Ammoniak/Toluol

3.6.6. Umsetzung von 2,6-Bis(2‘,6`-dimethylphenyl)phenylgermanium- 32 trichlorid mit $\mathrm{KOH} / \mathrm{NH}_{3}$; Darstellung von $\left[\left(\mathrm{R}^{*} \mathrm{Ge}\right)_{4} \mathrm{O}_{6}\right](\mathbf{1 9})$

3.7. Umsetzungen von Verbindungen mit niedervalenten Elementen 33 der 14. Gruppe

3.7.1. Darstellung und Struktur von (Dipp) ${ }_{2} \mathrm{NacNacAlHBH}_{2}(\mathbf{2 0}) \quad 33$

3.7.2. Versuch zur Darstellung von (Dipp) ${ }_{2} \mathrm{NacNacSnF}(\mathbf{1 6})$

3.7.3. Darstellung von $(t \text {-BuSn })_{4} \mathrm{~S}_{6}(\mathbf{2 1}) \quad 36$

4. Zusammenfassung 38

5. Experimenteller Teil 44

5.0.1. Allgemeine Arbeitstechniken 44

5.0.2. Physikalische Messmethoden 44

5.1. Umsetzungen von $\left[\operatorname{MeAIN}\left(2,6-i-\operatorname{Pr}_{2} \mathbf{C}_{6} \mathbf{H}_{3}\right)\right]_{3}(1) \quad 46$

5.1.1. Darstellung von $\left[\mathrm{FAlN}\left(2,6-i-\mathrm{Pr}_{2} \mathrm{C}_{6} \mathrm{H}_{3}\right)\right]_{3} \cdot 3$ THF (7) und $\left[\mathrm{Me}_{2} \mathrm{SnN}(2,6-\quad 46\right.$ $\left.\left.i-\mathrm{Pr}_{2} \mathrm{C}_{6} \mathrm{H}_{3}\right)\right]_{2}(\mathbf{8})$

5.1.2. Allgemeine Vorschrift zur Umsetzung von 1 mit $n-\mathrm{Bu}_{4}\left(\mathrm{HF}_{2}\right) \quad 48$

5.1.3. Umsetzung von 1 mit F 3 B. OEt $_{2}$

5.1.4. Darstellung von $\left[\mathrm{ClAlN}\left(2,6-i-\mathrm{Pr}_{2} \mathrm{C}_{6} \mathrm{H}_{3}\right)\right]_{3} \cdot 3 \mathrm{THF}(\mathbf{9}) \quad 48$

5.1.5. Darstellung von Darstellung von $\left[\operatorname{BrAlN}\left(2,6-i-\operatorname{Pr}_{2} \mathrm{C}_{6} \mathrm{H}_{3}\right)\right]_{3} \cdot 3$ THF (10) 49

$\begin{array}{ll}\text { 5.1.6. } & \text { Umsetzung von } \mathbf{1} \text { mit } \mathrm{I}_{3} \mathrm{~B} \\ & \end{array}$

5.1.7. Umsetzung von 1 mit $\mathrm{H}_{3} \mathrm{~B} \cdot \mathrm{THF}$, Darstellung von $[\operatorname{MeAlN}(2,6-i-\quad 51$ $\left.\left.\mathrm{Pr}_{2} \mathrm{C}_{6} \mathrm{H}_{3}\right)\right]_{3} \cdot 3$ THF (11)

5.1.8. Umsetzung von 1 mit $\mathrm{PhSi}(\mathrm{C} \equiv \mathrm{CH})_{3}$ 
5.2. Darstellung und Hydrolyse von monoorganylsubstituierten 52 Germaniumtrihalogeniden

5.2.1. Darstellung von 2,6-Bis(2`,6`-dimethylphenyl)-1-iodbenzol (12)

5.2.2. Darstellung von 2,6-Bis(2‘,6`-dimethylphenyl)phenylgermanium- 53 trichlorid (13)

5.2.3. Darstellung von 2,6-Bis(2‘,6`-dimethylphenyl)phenylgermanium- 54 tribromid (14)

5.2.4. Darstellung von 2,6-Bis(2‘,6`-dimethylphenyl)phenylgermanium- 55 triiodid (15)

5.2.5. Darstellung von 2,6-Bis(2‘,6`-dimethylphenyl)phenylgermanium- 56 trifluorid (16)

5.2.6. Darstellung von $\operatorname{DippN}\left(\mathrm{SiMe}_{3}\right) \mathrm{GeF}_{3}(\mathbf{1 7})$

$\begin{array}{lllll}\text { 5.3. } & \text { Versuche zur Hydrolyse von Monoorganylgermanium- } 57\end{array}$ trihalogeniden

5.3.1. Umsetzung von $\operatorname{DippN}\left(\mathrm{SiMe}_{3}\right) \mathrm{GeBr}_{3}$ mit $\mathrm{PhNH}_{2}$ und $\mathrm{H}_{2} \mathrm{O}$

5.3.2. Umsetzung von $\operatorname{DippN}\left(\mathrm{SiMe}_{3}\right) \mathrm{GeBr}_{3}$ mit $\mathrm{H}_{2} \mathrm{O}$ in Gegenwart von 58 Pyridin, Darstellung von [(DippN $\left.\left.\left(\mathrm{SiMe}_{3}\right) \mathrm{Ge}\right)_{4} \mathrm{O}_{6}\right](\mathbf{1 8})$

5.3.3. Umsetzung von $\operatorname{DippN}\left(\mathrm{SiMe}_{3}\right) \mathrm{GeBr}_{3}$ mit $\mathrm{H}_{2} \mathrm{O}_{2}$

5.3.4. Umsetzung von (DippN $\left.\left(\mathrm{SiMe}_{3}\right) \mathrm{Ge}\left(\mathrm{NH}_{2}\right)_{2}\right)_{2} \mathrm{NH}$ mit $\mathrm{H}_{2} \mathrm{O}$

5.3.5. Umsetzung von $\operatorname{DippN}\left(\mathrm{SiMe}_{3}\right) \mathrm{GeBr}_{3}$ mit $\mathrm{KOH}$ im Zwei- 60 Phasensystem Ammoniak/Toluol

5.3.6. Umsetzung von 2,6-Bis(2`,6`-dimethylphenyl)phenylgermanium- 61 trichlorid mit $\mathrm{KOH} / \mathrm{NH}_{3}$, Darstellung von $\left[\left(\mathrm{R}^{*} \mathrm{Ge}\right)_{4} \mathrm{O}_{6}\right](\mathbf{1 9})$ 
5.4. Umsetzungen von Verbindungen mit niedervalenten Elementen

der 14. Gruppe

5.4.1. Darstellung von (Dipp) ${ }_{2} \mathrm{NacNacAlHBH}_{2}(\mathbf{2 0})$

5.4.2. Versuch zur Darstellung von (Dipp) ${ }_{2} \mathrm{NacNacSnF}$

5.4.3. Darstellung von $(t-\mathrm{BuSn})_{4} \mathrm{~S}_{6}(\mathbf{2 1})$

5.5. Behandlung und Entsorgung der Abfälle 65

6.

Röntgenographischer Anhang

7. Literaturverzeichnis 


\section{Abkürzungsverzeichnis:}

\begin{tabular}{|c|c|}
\hline $\mathrm{Ar}, \mathrm{Ar}^{*}$ & aromatischer Rest \\
\hline arom. & aromatisch \\
\hline Ber. & berechnet \\
\hline$t-\mathrm{Bu}$ & Tertiärbutyl \\
\hline $\mathrm{Cp}$ & Cyclopentyl \\
\hline Dipp & 2,6-Diisopropylphenyl \\
\hline EI & Elektronenstoßionisation \\
\hline Et & Ethyl \\
\hline Gef. & gefunden \\
\hline $\mathrm{h}$ & Stunden \\
\hline $\mathrm{Hz}$ & Hertz \\
\hline IR & Infrarot \\
\hline$J$ & Kopplungskonstante \\
\hline$m$ & Masse \\
\hline M & Molekülionenpeak \\
\hline $\mathrm{Me}$ & Methyl \\
\hline $\mathrm{MHz}$ & Megahertz \\
\hline MS & Massenspektrogramm \\
\hline$n-$ & normal \\
\hline NMR & Nuclear Magnetic Resonance \\
\hline $\mathrm{Ph}$ & Phenyl \\
\hline ppm & parts per million \\
\hline$i-\operatorname{Pr}$ & Isopropyl \\
\hline $\mathrm{R}, \mathrm{R}^{6}$ & organischer Rest \\
\hline THF & Tetrahydrofuran \\
\hline TMS & Tetramethylsilan \\
\hline$z$ & Ladung \\
\hline$\delta$ & chemische Verschiebung \\
\hline$\tilde{v}$ & Wellenzahl \\
\hline
\end{tabular}




\section{Einleitung}

1.1. Organoaluminiumfluoride

Aluminiumtrichlorid ist ein gängiges Edukt für die Darstellung von Aluminiumverbindungen, was mit der guten Löslichkeit in koordinierenden organischen Lösungsmitteln und dem niedrigen Schmelzpunkt erklärt werden kann. ${ }^{[1]}$ Alumuminiumtrifluorid hat verglichen damit einen um $1000{ }^{\circ} \mathrm{C}$ höheren Schmelzpunkt und ist wesentlich unreaktiver. Ferner ist die Aluminium-Fluor-Bindung $(532 \mathrm{~kJ} / \mathrm{mol})$ eine der thermodynamisch stabilsten überhaupt, ${ }^{[2]}$ weshalb direkte Reaktionen mit metallierten organischen Liganden nicht möglich sind. ${ }^{[3]}$

Aus diesen Gründen waren in organischen Lösungsmitteln lösliche Organoaluminiumfluoride bis in die 50er Jahre des letzten Jahrhunderts nahezu unbekannt. Als erste berichteten Ziegler et al. 1955 über die Darstellung neutraler Organoaluminiumfluoride, synthetisiert durch Metathese von Organoaluminiumchloriden mit NaF. ${ }^{[4-7]}$ In einem Patent von 1957 berichtete Ziegler von der Darstellung des $\mathrm{R}_{2} \mathrm{AlF}$, ohne näher auf die spektroskopische Charakterisierung der erhaltenen Verbindung einzugehen. ${ }^{[5]}$ In weiteren Patenten stellte er weitere Organoaluminiumfluoride bzw, Diorganoaluminiumfluoride vor. ${ }^{[8,9]}$

Andere Möglichkeiten, Organoaluminiumfluoride darzustellen, sind Umsetzungen von Alkylaluminium-Verbindungen mit $\mathrm{BF}_{3} \cdot \mathrm{OEt}_{2},{ }^{[10,11]} \mathrm{SiF}_{4}, \mathrm{R}_{2} \mathrm{SiF}_{2}$, $\mathrm{ZnF}_{2}, \mathrm{CdF}_{2}, \mathrm{SbF}_{3}, \mathrm{PbF}_{2}{ }^{[12]}$ Auch mit Fluorierungsmitteln wie $\mathrm{XeF}_{2}{ }^{[13]}$ und Laserphotosenstiviertem $\mathrm{SF}_{6}{ }^{[14]}$ sind sie erhältlich. ${ }^{[15]}$

Vor einigen Jahren wurde im Arbeitskreis Roesky $\mathrm{Me}_{3} \mathrm{SnF}$ zur Darstellung von Fluorverbindungen der Gruppen 4 - 6 und Hauptgruppenfluoriden eingeführt. ${ }^{[16-21]}$ Der Vorteil dieses Reagenzes ist, dass der Verlauf der Reaktion durch „Auflösen” des in organischen Lösungsmitteln schlecht löslichen $\mathrm{Me}_{3} \mathrm{SnF}$ beobachtet werden kann und dass $\mathrm{Me}_{3} \mathrm{SnF}$ als selektives Fluorierungsreagenz wirkt. Werden wie z. B. im Falle von Fluoriden der Gruppe 4, die entsprechenden Chloride verwendet, so ist es möglich, das bei der Reaktion entstandene $\mathrm{Me}_{3} \mathrm{SnCl}$ wieder zu recyclen oder durch Reaktion mit $\mathrm{NaF}$ wieder in das Fluorid zu überführen. ${ }^{[16-21]}$ 
Auf diesen Erfahrungen aufbauend gelang es in unserem Arbeitskreis, monound difluorierte Aminoalane ${ }^{[22]}$ und Trisylaluminiumdifluoride $\left(\text { Trisyl }=\left(\mathrm{Me}_{3} \mathrm{Si}\right)_{3} \mathrm{C}-\right)^{[23]}$ darzustellen. Die Fluoride sind durch Metathesereaktion aus den entsprechenden Dimethylverbindungen des Aluminiums erhältlich. (Schema 1.)

$\mathrm{RAlMe}_{2}+2 \mathrm{Me}_{3} \mathrm{SnF} \underset{-2 \mathrm{Me}_{4} \mathrm{Sn}}{\longrightarrow}$

$1 / 3$

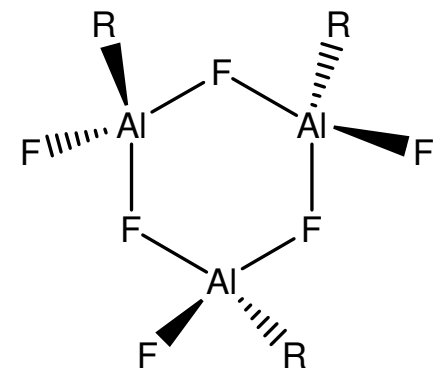

$\mathrm{R}=\operatorname{DippN}\left(\mathrm{SiMe}_{3}\right)^{[22]} \mathrm{bzw}$.

$\mathrm{R}=\left(\mathrm{Me}_{3} \mathrm{Si}\right)_{3} \mathrm{C}^{[23]}$

\section{Schema 1.}

Das bei der Reaktion entstehende Tetramethylstannan ist leicht flüchtig und somit einfach von den Produkten zu trennen. Die Difluoride liegen in nichtkoordinierenden Lösungsmitteln als fluorverbrückte Trimere vor, während die Dimethylalane als Dimere auftreten. ${ }^{[22,23]}$ Darüber hinaus weisen sie ein äusserst interessantes Reaktionsverhalten auf: Durch THF-Spaltung konnte die Bildung von $\mathrm{Al}_{6} \mathrm{~F}_{12}$-Tetrakishexaedern mit einem Sauerstoffatom im Zentrum beobachtet werden. ${ }^{\text {[24] }}$ In Anlehnung an Pearsons Konzept der harten und weichen Säuren und Basen ${ }^{[25,26]}$ wurde der Komplex $\left.\left[(\mathrm{PhMe})_{3} \mathrm{Ag}\right]^{+}\left[\left\{\left(\left(\mathrm{Me} \mathrm{Si}_{3}\right)_{3} \mathrm{CAl}\right)_{2} \mathrm{~F}_{5}\right]_{2}\right\} \mathrm{Li}\right]^{-}$erhalten und strukturell charakterisiert. ${ }^{[27]}$ Darüber hinaus gelang die Darstellung des Komplexes $\left[\mathrm{Cp}_{2} \mathrm{ZrMe}\right](\mu-$ $\mathrm{F})\left[\mathrm{F}_{2} \mathrm{AlC}\left(\mathrm{SiMe}_{3}\right)_{3}\right] .{ }^{[28]}$ Eine mögliche Anwendung für solche Verbindungen besteht in der Olefinpolymerisation als Ziegler-Natta-Katalysatoren. ${ }^{[29,30]}$

Eine weitere Möglichkeit, Fluoratome einzuführen, ist die Umsetzung mit $\left(\mathrm{Me}_{2} \mathrm{AlF}\right)_{4}$. So konnte durch Umsetzung mit $\mathrm{DippNH}_{2}$ ein achtatomiger Ring mit fluorverbrückten Aluminiumzentren erhalten werden. ${ }^{[31]}$ Die anschließende Pyrolyse ergab unter Eliminierung von $\mathrm{DippNH}_{2}$ ein Heterokuban. ${ }^{[32]}$ 


\subsection{Terphenyle und Vinamidine als Ligandensysteme}

Terphenyle und (Dipp) ${ }_{2} \mathrm{NacNac}$ als Ligandensysteme stehen seit Jahren im Mittelpunkt einer Vielzahl von Untersuchungen. Ein Grund liegt in der Eigenschaft dieser Gruppen Elemente in ungewöhnlichen Oxidationsstufen bzw. Doppelbindungssysteme der schwereren Elemente zu stabilisieren. ${ }^{[33-37]}$ So gelang Power et al. die Stabilisierung von Verbindungen mit Doppelbindungen schwerer Elemente der 15 Gruppe (Arsen, Antimon und Bismut). ${ }^{[38]}$ Auch mit Elementen der Gruppe 14 wie Germanium, Zinn und Blei konnten Verbindungen dargestellt werden, die interessante Bindungsverhältnisse enthalten. Hier sind insbesondere Alkin-analoge Verbindungen von Interesse, in welchen der Kohlenstoff durch die schwereren Homologen wie Germanium, Zinn und Blei ersetzt ist. Erhältlich waren auf diese Weise 2,6-Trip ${ }_{2} \mathrm{H}_{3} \mathrm{C}_{6} \mathrm{PbPbC}_{6} \mathrm{H}_{3}$-2,6-Trip 2 (Trip = 2,4,6-i- $\mathrm{Pr}_{3} \mathrm{C}_{6} \mathrm{H}_{2}$ ), ${ }^{[39]} \mathrm{Dipp}_{2} \mathrm{H}_{3} \mathrm{C}_{6} \mathrm{SnSnC}_{6} \mathrm{H}_{3}$ 2,6-Dipp $2\left(\text { Dipp }=2,6-i-\operatorname{Pr}_{2} \mathrm{C}_{6} \mathrm{H}_{3}\right)^{[40]}$ und 2,6-Dipp ${ }_{2} \mathrm{H}_{3} \mathrm{C}_{6} \mathrm{GeGeC}_{6} \mathrm{H}_{3}-2,6-\mathrm{Dipp}_{2}$ (Dipp = 2,6-i- $\left.\mathrm{Pr}_{2} \mathrm{C}_{6} \mathrm{H}_{3}\right),{ }^{[41]}$ die auch strukturell charakterisiert werden konnten. Dadurch konnten wichtige Aspekte über die Natur solcher Bindungen bei Hauptgruppenelementen gewonnen werden. ${ }^{[42-46]}$ Gemein ist den Verbindungen, dass die Liganden transgebunden (Schema 2) und die Metall-Metall-Bindungslängen kürzer als Einfachbindungen sind.

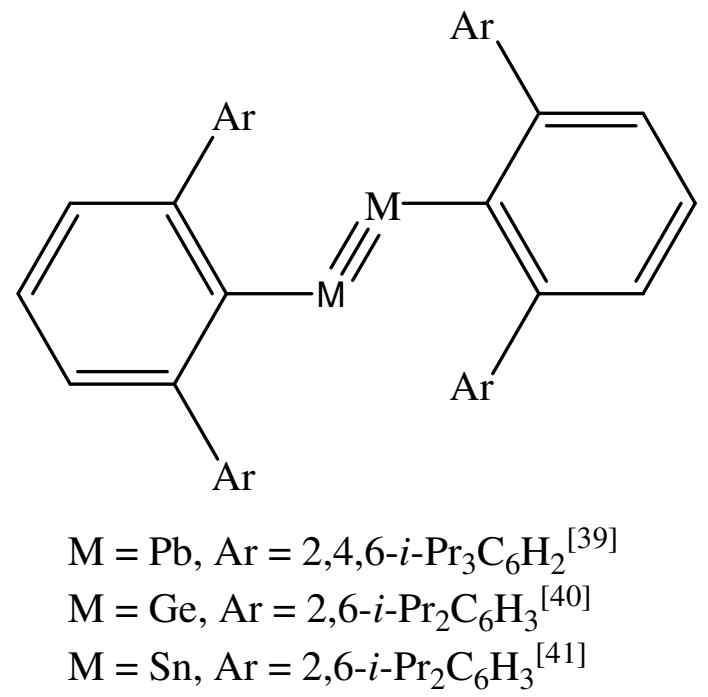

Schema 2. Alkinanaloge Verbindungen der schwereren Elemente der 14. Gruppe 
Wie die Terphenyle erlangten in den letzten Jahren Vinamidine wie (Dipp) ${ }_{2} \mathrm{NacNac} \quad\left(=2-\left[\left(2,6-i-\mathrm{Pr}_{2} \mathrm{C}_{6} \mathrm{H}_{3}\right) \mathrm{NH}\right)-4-\left(\left(2,6-i-\mathrm{Pr}_{2} \mathrm{C}_{6} \mathrm{H}_{3}\right) \mathrm{N}\right] \mathrm{C}_{6} \mathrm{H}_{7}\right)$ als Liganden aufgrund ihres sterischen Anspruches als auch ihrer chelatisierenden Eigenschaften zunehmende Bedeutung in der Organometallchemie. ${ }^{[47-56]}$ Vinamidine sind in der Lage, niedrige Oxidationsstufen von Elementen der 13. Gruppe zu stabilisieren. Kürzlich gelang in unserem Arbeitskreis die Darstellung von (Dipp) ${ }_{2} \mathrm{NacNacAl}(\mathrm{I})$, dem ersten Beispiel einer bei Raumtemperatur stabilen monomeren Aluminium(I)-Verbindung. ${ }^{[57]}$ Ein weiteres interessantes Beispiel für Vinamidin-Komplexe ist ein Bor-MagnesiumVinamidin Komplex, in welchem das Magnesium sechs- und achtfach koordiniert ist. ${ }^{[58]}$ Vor kurzem beschrieben Power et al. die Synthese und strukturelle Charakterisierung der ersten Verbindung mit Gallium-Stickstoff-Doppelbindung, wobei sowohl das Vinamidin als auch das Trip-Terphenyl als stabilisierende Ligandensysteme Verwendung fanden. ${ }^{[59]}$ (Schema 3.)

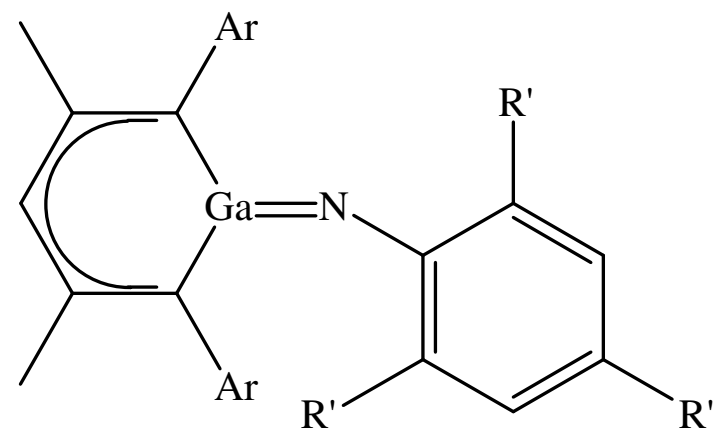

$$
\begin{aligned}
& \mathrm{Ar}=2,6-i-\mathrm{Pr}_{2} \mathrm{C}_{6} \mathrm{H}_{3} \\
& \mathrm{R}^{\prime}=2,4,6-i-\mathrm{Pr}_{3} \mathrm{C}_{6} \mathrm{H}_{2}
\end{aligned}
$$

Schema 3. Darstellung der ersten Verbindung mit einer Ga-N-Doppelbindung

Ferner gelang die strukturelle Charakterisierung von Verbindungen mit einer formalen Doppelbindung zwischen halogensubstituierten Elementen der Gruppe 14 und $16\left((\mathrm{Dipp})_{2} \mathrm{NacNacM}(=\mathrm{E}) \mathrm{X}\right.$ mit $\mathrm{M}=\mathrm{Ge}, \mathrm{Sn} ; \mathrm{E}=\mathrm{S}, \mathrm{Se}, \mathrm{Te} ; \mathrm{X}=$ Halogen $)$. Durch anschließende Umsetzung mit Organyllithium-Verbindungen sind die entsprechenden Organoderivate erhältlich, ohne dass zusätzlich ein sterisch anspruchsvoller Ligand eingeführt werden muss. ${ }^{[60]}$ 


\subsection{Organasilanole und -germanole}

Organosilanole sind seit langem bekannt ${ }^{[61-63]}$ und haben insbesondere als Siloxane eine Vielzahl von Anwendungsmöglichkeiten. ${ }^{[64-67]}$ In den letzten Jahren konnte die Chemie der Silanole in unserem Arbeitskreis durch die Einführung kondensationsstabiler und in gängigen Lösungsmitteln löslicher Silantriole erweitert werden, ${ }^{[68,69]}$ darstellbar durch Hydrolyse der entsprechenden Organotrichlorosilane in Anwesenheit einer Base. ${ }^{[68,69]}$ Durch Reaktion mit einfachen Metallalkylen bzw. hydriden ließen sich unter Alkan- bzw. Wasserstoffeliminierung zahlreiche molekulare Modellverbindungen für zeolithische Materialen synthetisieren und strukturell charakterisieren. ${ }^{[70-72]}$

Im Gegensatz dazu sind nur wenige Beispiele ${ }^{[73-79]}$ für Germanole bekannt. Ein Grund hierfür ist die höhere Tendenz zur Selbstkondensation. ${ }^{[74,80]}$ Puff et al. gelang durch Hydrolyse von $t-\mathrm{Bu}_{2} \mathrm{GeCl}_{2}$ die Darstellung des Germandiols $t-\mathrm{Bu} 2 \mathrm{Ge}(\mathrm{OH})_{2},{ }^{[80]}$ durch Dehydratisierung konnten sie das Produkt $\left(t-\mathrm{Bu}_{2} \mathrm{GeO}\right)_{3}$ erhalten. Im Arbeitskreis Jurkschat konnte durch Hydrolyse von $t$ - $\mathrm{Bu}_{2} \mathrm{Ge}(\mathrm{OEt})_{2}$ das Germandiol $t-\mathrm{Bu}_{2} \mathrm{Ge}(\mathrm{OH})_{2}$ und dessen Kondensationsprodukt $\left(t-\mathrm{Bu}_{2} \mathrm{GeOH}\right)_{2} \mathrm{O}$ dargestellt werden. ${ }^{[78]}$ Power et al. beobachteten bei der Umsetzung von $\mathrm{R}_{2} \mathrm{Ge}\left(\mathrm{R}=2,6-\mathrm{Mes}_{2} \mathrm{C}_{6} \mathrm{H}_{3}\right)$ mit $\mathrm{N}_{2} \mathrm{O}$ bzw. $\mathrm{Me}_{3} \mathrm{NO}$ die Weiterreaktion des entstandenem Germanons durch Spuren von $\mathrm{H}_{2} \mathrm{O}$ zum Germandiol $\mathrm{R}_{2} \mathrm{Ge}(\mathrm{OH})_{2}{ }^{[81]}$

\subsection{Sesquichalcogenide}

Organosubstituierte Sesquichalcogenide mit Elementen der 14. Gruppe $(\mathrm{RE})_{2 \mathrm{n}} \mathrm{Y}_{3 \mathrm{n}}(\mathrm{E}=\mathrm{Si}, \mathrm{Ge}, \mathrm{Sn} ; \mathrm{Y}=\mathrm{O}, \mathrm{S}, \mathrm{Se})$ sind seit langem sowohl von wissenschaftlichem als auch von anwendungstechnischem Interesse. ${ }^{[82-85]}$ Ein besonderer Weg Sesquichalcogenide darzustellen, ist die Umsetzung von $t$ - $\mathrm{BuGeCl}_{3}$ mit $\left(\mathrm{NH}_{4}\right)_{2} \mathrm{~S}_{5}$, dabei entstand ein polyedrisches Gerüst mit „Doppeldecker”-Struktur. (Schema 4.) Weiteres Erhitzen dieser Verbindung ergab eine Verbindung mit adamantanartigem Gerüst. ${ }^{[85]}$ 
<smiles>CC(C)(C)[Mg][As](C)(N)C(C)(C)C</smiles>

Schema 4.

Im Arbeitskreis Matsumoto wurde $\operatorname{Li}_{2} X(X=S$, Se $)$ eingesetzt, um Sesquichalcogenide darzustellen. ${ }^{[84]}$ Auch bei diesen Reaktionen entstanden sowohl adamantanartige- als auch „Doppeldecker”-Strukturen. Einen anderen Weg beschrieben Roesky et al.. Hierbei wurde Schwefel bzw. Selen in situ durch Ammoniak zu den entsprechenden Sulfiden bzw. Seleniden reduziert. ${ }^{[86]}$ 


\section{Aufgabenstellung}

Ziel dieser Arbeit war die vollständige Fluorierung, Chlorierung, Bromierung und Iodierung eines Iminoalans und mögliche Charakterisierung aller dabei entstehenden Produkte.

Ein weiteres Ziel war die Darstellung von monoorganylsubstituierten Germaniumhalogeniden. Durch Hydrolyse sollte die Synthese von stabilen Germanolen untersucht werden.

Ebenfalls von Interesse war es, das Reaktionsverhalten von Verbindungen mit niedervalenten Elementen der Gruppe 14 zu untersuchen. 


\section{Theoretischer Teil}

\subsection{Fluorierung des Alumazens $\left[\mathrm{MeAIN}\left(2,6-i-\operatorname{Pr}_{2} \mathrm{C}_{6} \mathrm{H}_{3}\right)\right]_{3}(1)$ mit $\mathrm{Me}_{3} \mathrm{SnF}$}

Das von Power et al. 1988 dargestellte Alumazen $\left[\mathrm{MeAlN}\left(2,6-i-\mathrm{Pr}_{2} \mathrm{C}_{6} \mathrm{H}_{3}\right)\right]_{3}$ (1), ${ }^{[87]}$ welches durch Umsetzung mit $\mathrm{DippNH}_{2}$ und $\mathrm{Me}_{3} \mathrm{Al}$ (Schema 6) in Toluol in zwei Reaktionsschritten dargestellt werden kann, gilt auch mehr als 15 Jahre nach seiner Entdeckung als einziges trimeres Iminoalan. Es ist strukturell ähnlich aufgebaut wie das Borazin und gilt als „Quasiaromat”. ${ }^{[88]}$ Sein besonderes Reaktionsverhalten kann auf die hohe Polarität der Aluminium-Stickstoff-Bindung zurückgeführt werden. Die Substituenten stabilisieren sowohl das elektronisch als auch das koordinativ ungesättigte Ringsystem.

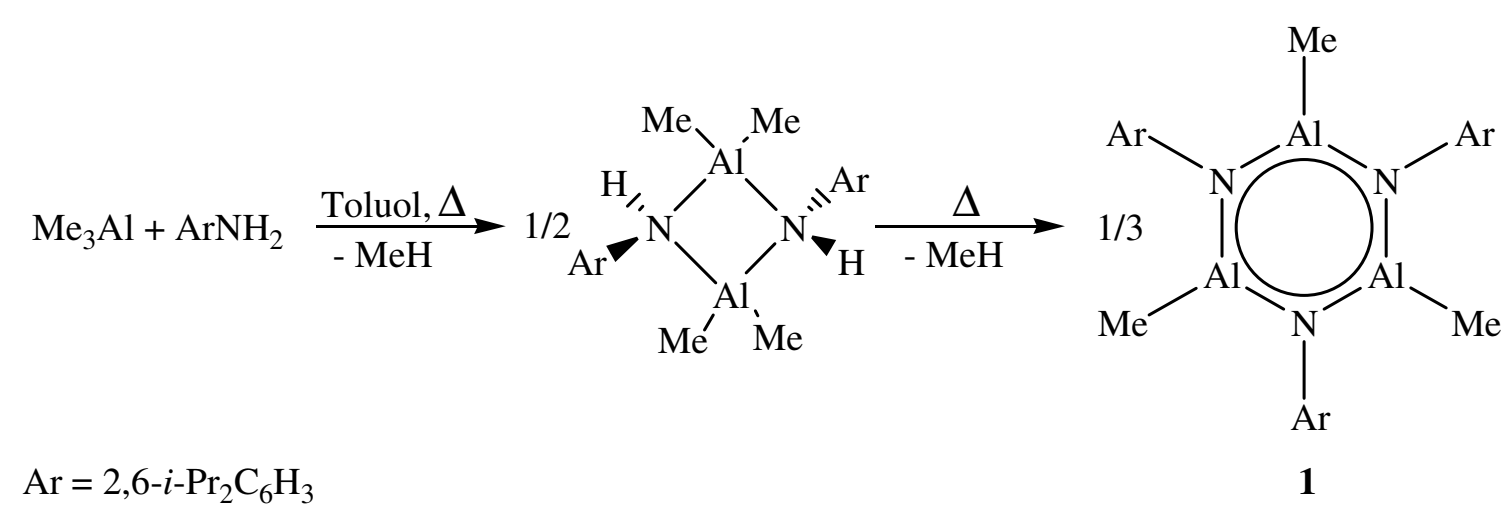

Schema 6. Darstellung von $\left[\mathrm{MeAlN}\left(2,6-i-\mathrm{Pr}_{2} \mathrm{C}_{6} \mathrm{H}_{3}\right)\right]_{3}(\mathbf{1})$

Im Arbeitskreis wurde das außergewöhnliche Reaktionsverhalten von $\mathbf{1}$ untersucht. Es konnte u.a. gezeigt werden, dass die dreifach-koordinierten Lewis-sauren Aluminiumzentren mit fluorhaltigen Verbindungen der 4. Gruppe stabile LewisSäure/Base-Komplexe ausbilden können. So gelang durch die Umsetzung von 1 mit $\mathrm{CpTiF}_{3}{ }^{[89]}\left(\mathrm{Me}_{3} \mathrm{Si} \mathrm{C}_{5} \mathrm{H}_{4} \mathrm{TiF}_{3}{ }^{[90]}\right.$ bzw. $\left(\mathrm{Me}_{3} \mathrm{Si}_{2}\right)_{2} \mathrm{C}_{5} \mathrm{H}_{3} \mathrm{ZrF}_{3}{ }^{[89]}$ die Bildung der heteroatomaren adamantanartigen Verbindungen 2, 3 bzw. 4 (Schema 7), wobei zwei Stickstoffatome und ein Fluoratom jeweils ein Aluminiumatom mit dem Atom der 4. Gruppe miteinander verknüpfen. 
<smiles>C[Al]1N([Al])[Al](C)N([Al])[Al]([Al])N1[Al]</smiles>

1

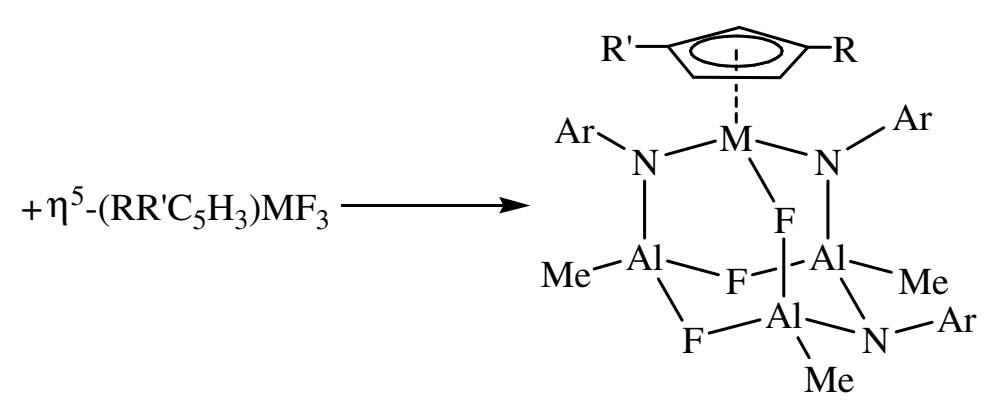

2: $\mathrm{R}, \mathrm{R}^{\prime}=\mathrm{H} ; \mathrm{M}=\mathrm{Ti}$

3: $\mathrm{R}=\mathrm{Me}_{3} \mathrm{Si}, \mathrm{R}^{\prime}=\mathrm{H} ; \mathrm{M}=\mathrm{Ti}$

4: $\mathrm{R}, \mathrm{R}^{\prime}=\mathrm{Me}_{3} \mathrm{Si} ; \mathrm{M}=\mathrm{Zr}$ $\mathrm{Ar}=2,6-i-\mathrm{Pr}_{2} \mathrm{C}_{6} \mathrm{H}_{3}$

Schema 7.

Die Umsetzung von 1 mit $\mathrm{Cp}^{*} \mathrm{TiF}_{3}$ führte ebenfalls zur Ausbildung eines heteroatomaren adamantanartigen Gerüstes, hier allerdings verbrücken zwei Fluoratome und ein Stickstoffatom die Metallzentren. ${ }^{[90]}$

Mit $\mathrm{Me}_{3} \mathrm{SnF}$ ist es möglich, selektiv zu fluorieren. Durch selektive Fluorierung von 1 waren bereits die entsprechenden Monofluoro- 5 bzw. Difluoro-Derivate 6 erhältlich. ${ }^{[91,92]}$ (Schema 8)

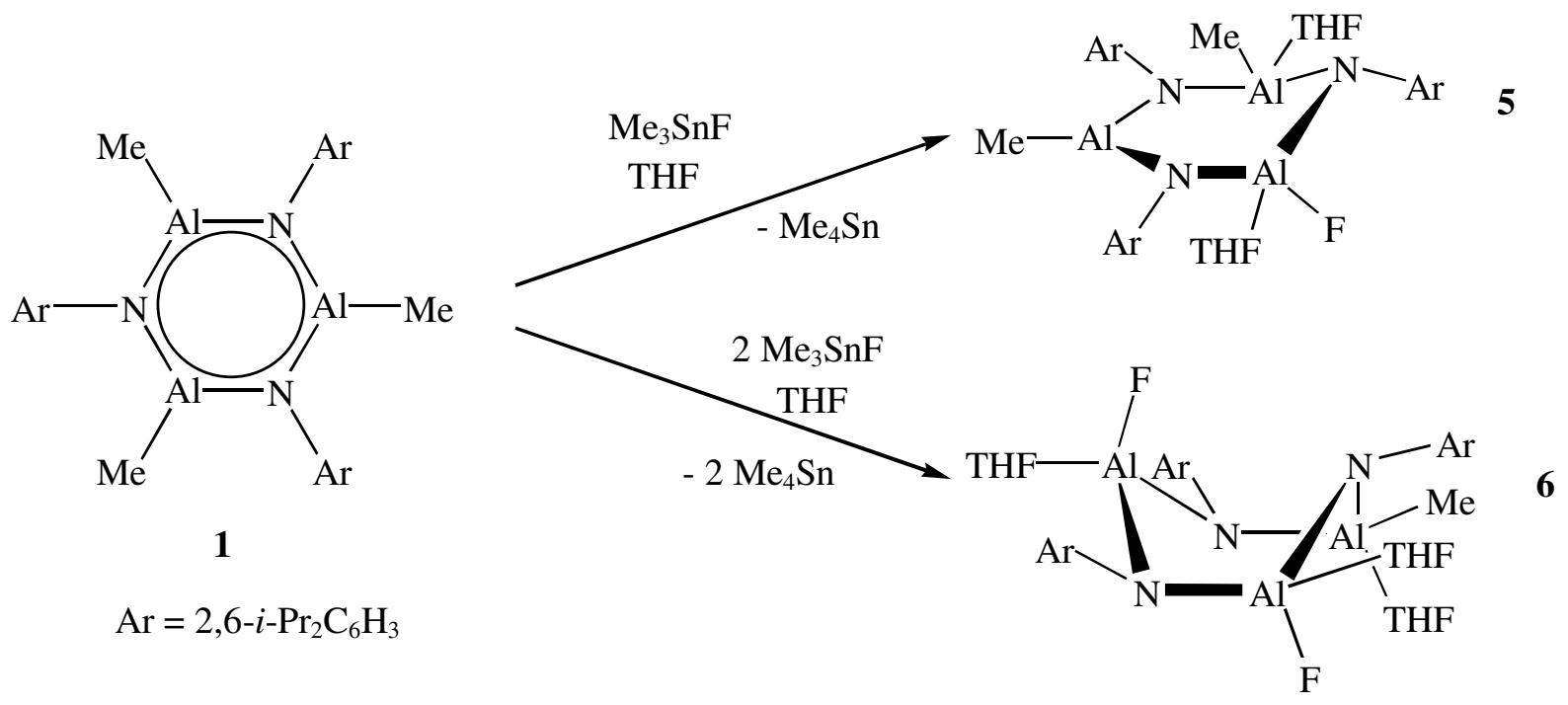

Schema 8. 


\subsubsection{Darstellung von $\left[\mathrm{FAIN}\left(2,6-i-\mathrm{Pr}_{2} \mathrm{C}_{6} \mathrm{H}_{3}\right)\right]_{3} \cdot 3 \mathrm{THF}$ (7) und $\left[\mathrm{Me}_{2} \mathrm{SnN}(2,6-i-\right.$ $\left.\left.\operatorname{Pr}_{2} \mathrm{C}_{6} \mathrm{H}_{3}\right)\right]_{2}(8)$}

Analog zur Darstellung des Mono- bzw. Difluoridss ${ }^{[91]}$ wurde eine Suspension aus $\mathrm{Me}_{3} \mathrm{SnF}$ in THF mit einer Lösung aus 1 in THF versetzt und 24 Stunden lang bei Raumtemperatur gerührt. Wie bei der Darstellung der Trisylalane ${ }^{[23]}$ und von $\mathbf{5}$ und $\mathbf{6}$ war ein koordinierendes Lösungsmittel nötig, da die Fluorierung in nichtkoordinierenden Lösungsmitteln ( $n$-Hexan, Toluol) zu Verbindungen führten, die nicht näher charakterisiert werden konnten. Das unlösliche $\mathrm{Me}_{3} \mathrm{SnF}$ reagierte dabei vollständig ab. (Schema 9) Nach Entfernen des Lösungsmittels im Vakuum und Waschen mit $n$-Hexan wurde ein Gemisch aus $\mathbf{7}$ und $\mathbf{8}$ erhalten.

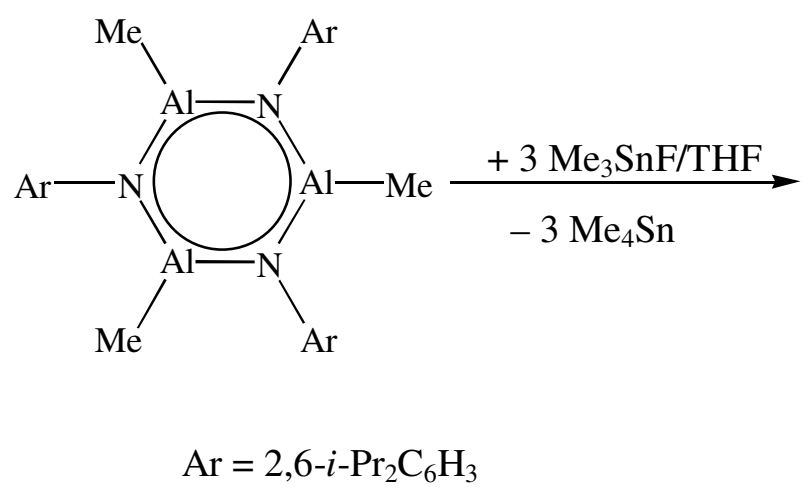

1
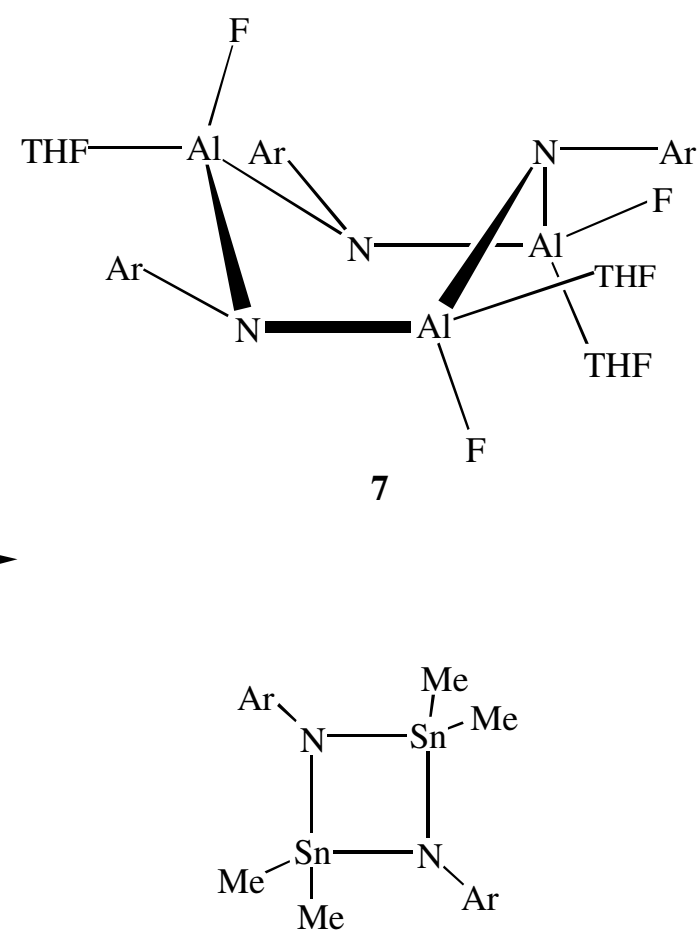

8

Schema 9.

Zusammensetzung und Konstitution von $\mathbf{7}$ und $\mathbf{8}$ konnten spektroskopisch ermittelt werden. Außerdem konnten von 7 und 8 Einkristall-Röntgenstrukturanalysen durchgeführt werden. 
Das ${ }^{1} \mathrm{H}$-NMR-Spektrum von 7 in $\mathrm{C}_{6} \mathrm{D}_{6}$ ähnelt denen von 5 und $\mathbf{6}$, wobei die Resonanzen für an Aluminium-gebundene Methylgruppen vollständig verschwunden sind. Im ${ }^{19}$ F-NMR Spektrum zeigen die Fluoratome zwei scharfe Singuletts bei $\delta-166.3$ und -153.9 ppm in einem Intensitätsverhältnis von 2 : 1. Die korrekte Zusammensetzung von 7 wurde durch die Elementaranalyse bestätigt.

\subsubsection{Diskussion der Einkristall-Röntgenstrukturanalyse von 7}

Zur Anfertigung einer Röntgenstrukturanalyse geeignete Kristalle von 7 wurden durch Kristallisation aus THF/n-Hexan (1:5) bei $-26{ }^{\circ} \mathrm{C}$ erhalten. In Abb.1a ist das Ringgerüst von 7 ohne Ar-Reste und nur die Sauerstoffatome der THF-Moleküle und in Abb. 1b die Struktur von 7 dargestellt, ausgewählte Bindungslängen und -winkel enthält Tabelle 1.

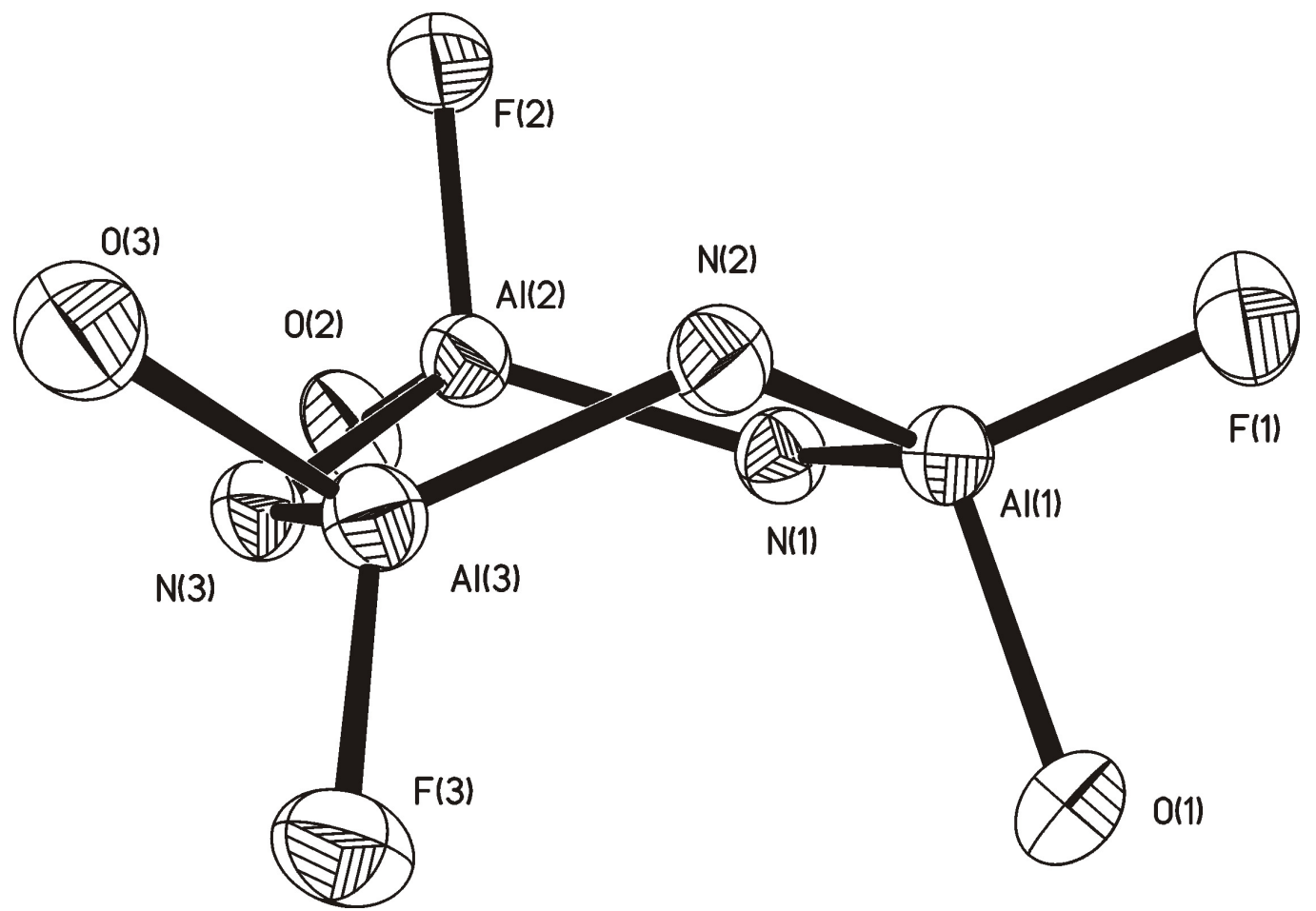

Abb.1a. Ringgerüst von 7 


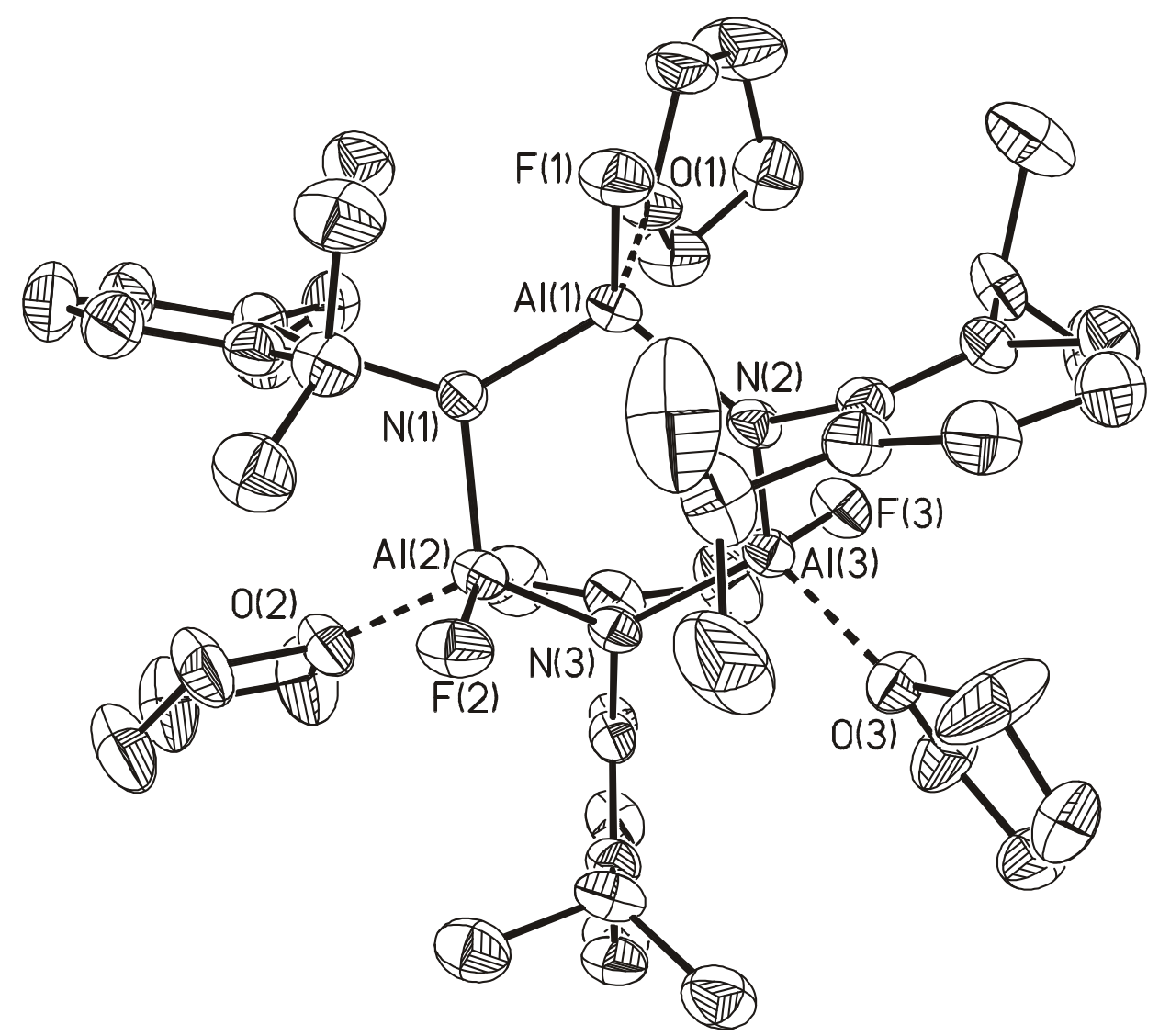

Abb.1b. Struktur von 7 im Kristall.

Tab.1. Ausgewählte Bindungslängen $[\AA]$ und -winkel $\left[{ }^{\circ}\right]$ von 7

\begin{tabular}{llll}
\hline $\mathrm{Al}(1)-\mathrm{F}(1)$ & $1.6798(17)$ & $\mathrm{F}(1)-\mathrm{Al}(1)-\mathrm{N}(1)$ & $119.64(10)$ \\
$\mathrm{Al}(2)-\mathrm{F}(2)$ & $1.6821(17)$ & $\mathrm{F}(1)-\mathrm{Al}(1)-\mathrm{N}(2)$ & $114.07(10)$ \\
$\mathrm{Al}(3)-\mathrm{F}(3)$ & $1.6879(16)$ & $\mathrm{F}(2)-\mathrm{Al}(2)-\mathrm{N}(1)$ & $111.97(9)$ \\
$\mathrm{Al}(1)-\mathrm{N}(1)$ & $1.808(2)$ & $\mathrm{F}(2)-\mathrm{Al}(2)-\mathrm{N}(3)$ & $117.46(10)$ \\
$\mathrm{Al}(1)-\mathrm{N}(2)$ & $1.833(2)$ & $\mathrm{F}(3)-\mathrm{Al}(3)-\mathrm{N}(2)$ & $114.79(9)$ \\
$\mathrm{Al}(2)-\mathrm{N}(1)$ & $1.831(2)$ & $\mathrm{F}(3)-\mathrm{Al}(3)-\mathrm{N}(3)$ & $114.33(9)$ \\
$\mathrm{Al}(2)-\mathrm{N}(3)$ & $1.818(2)$ & $\mathrm{Al}(1)-\mathrm{N}(1)-\mathrm{Al}(2)$ & $113.84(12)$ \\
$\mathrm{Al}(3)-\mathrm{N}(2)$ & $1.813(2)$ & $\mathrm{Al}(1)-\mathrm{N}(2)-\mathrm{Al}(3)$ & $119.94(11)$ \\
$\mathrm{Al}(3)-\mathrm{N}(3)$ & $1.815(2)$ & $\mathrm{Al}(2)-\mathrm{N}(3)-\mathrm{Al}(3)$ & $114.51(11)$ \\
$\mathrm{Al}(1)-\mathrm{O}(1)$ & $1.912(2)$ & $\mathrm{N}(1)-\mathrm{Al}(1)-\mathrm{N}(2)$ & $110.88(10)$ \\
\hline
\end{tabular}




\begin{tabular}{lccc}
\hline $\mathrm{Al}(2)-\mathrm{O}(3)$ & $1.906(2)$ & $\mathrm{N}(1)-\mathrm{Al}(2)-\mathrm{N}(3)$ & $114.69(10)$ \\
$\mathrm{Al}(3)-\mathrm{O}(3)$ & $\mathrm{N}(2)-\mathrm{Al}(3)-\mathrm{N}(3)$ & $110.39(10)$ \\
& & $\mathrm{O}(1)-\mathrm{Al}(1)-\mathrm{F}(1)$ & $95.09(8)$ \\
& $\mathrm{O}(2)-\mathrm{Al}(2)-\mathrm{F}(2)$ & $93.86(9)$ \\
& $\mathrm{O}(3)-\mathrm{Al}(3)-\mathrm{F}(3)$ & $93.92(9)$ \\
\hline
\end{tabular}

7 kristallisiert monoklin in der Raumgruppe $P 2(1) / n$ mit vier unabhängigen Formeleinheiten pro Elementarzelle. Die zentrale Einheit dieser Verbindung besteht aus einem alternierend aus Aluminium- und Stickstoffatomen aufgebauten sechsgliedrigen $\mathrm{Al}_{3} \mathrm{~N}_{3}$-Ring in einer Bootkonfiguration. In dieser Konfiguration können alle Arylreste die bevorzugte äquatoriale Position einnehmen. Zwei der terminal gebundenen Fluoratome befinden sich über und ein Fluoratom unterhalb der Ringebene. Durch Koordination von THF verliert der Ring seine Planarität und somit sind die AluminiumStickstoff-Bindungen länger als die in $\mathbf{1}^{[87]}$ Die Aluminiumatome binden jeweils an zwei Stickstoffatome und an ein Fluoratom. Zusätzlich koordiniert ist jeweils ein Sauerstoffatom vom THF, dadurch besitzen die Aluminiumatome eine verzerrt tetraedrische Umgebung. Die Aluminium-Fluor-Bindungslängen in 7 reichen von 1.680 - $1.688 \AA$ und sind somit etwas länger als in $\left[\left\{\left(2,6-i-\mathrm{Pr}_{2} \mathrm{C}_{6} \mathrm{H}_{3}\right) \mathrm{N}\left(\mathrm{SiMe}_{3}\right) \mathrm{AlF}_{2}\right\}_{3}\right](1.634$ - $1.642 \AA)^{[22]}$ und $\mathrm{AlF}_{3}(1.63 \AA),{ }^{[93]}$ aber vergleichbar mit denen in $\left[\left(\mathrm{Me}_{3} \mathrm{Si}\right)_{3} \mathrm{CAlF}_{2}\right]_{3}$ $(1.657-1.681 \AA)^{[23]}$. Im Vergleich mit $\left[\operatorname{Tmp}_{2} \mathrm{Al}(\mu-\mathrm{F})\right]_{2}(\mathrm{Tmp}=$ Tetramethylpiperidin $)$ $(1.829-1.835 \AA)^{[94]}$ sind sie wesentlich kürzer. Die Aluminium-SauerstoffBindungslängen sind etwas länger, als sie in der Literatur publiziert sind. ${ }^{[23,95,96]}$

\subsubsection{Diskussion der Einkristall-Röntgenstrukturanalyse von 8}

Wurde das Gemisch in THF/Toluol (1:10) gelöst, kristallisierte bei $0{ }^{\circ} \mathrm{C} 8$ aus. Offensichtlich ist $\mathbf{8}$ ein Nebenprodukt aus der Reaktion von 7 mit $\mathrm{Me}_{3} \mathrm{SnF}$. Im ${ }^{1} \mathrm{H}-\mathrm{NMR}$ Spektrum findet man bei $\delta 0.40$ ppm die Resonanz für die Methylprotonen am Zinn. Die anderen Resonanzen können den Protonen des DippN-Liganden zugeordnet werden. Im ${ }^{119}$ Sn-NMR Spektrum wird das Signal für die Zinnatome bei $\delta 114.67$ ppm gefunden. 
Im EI-Massenspektrum von $\mathbf{8}$ erscheint der Molekülionen-Peak mit korrekter Isotopenverteilung bei m/z 648 und einer Intensität von 40. Das Signal mit der größten Intensität kann dem Dipp-Rest zugeordnet werden. Die Zusammensetzung von 8 wird durch die korrekte Elementaranalyse bestätigt. Abb. 2 zeigt die Struktur von 8 im Kristall. Ausgewählte Bindungslängen und -winkel sind in Tab. 2 aufgelistet.

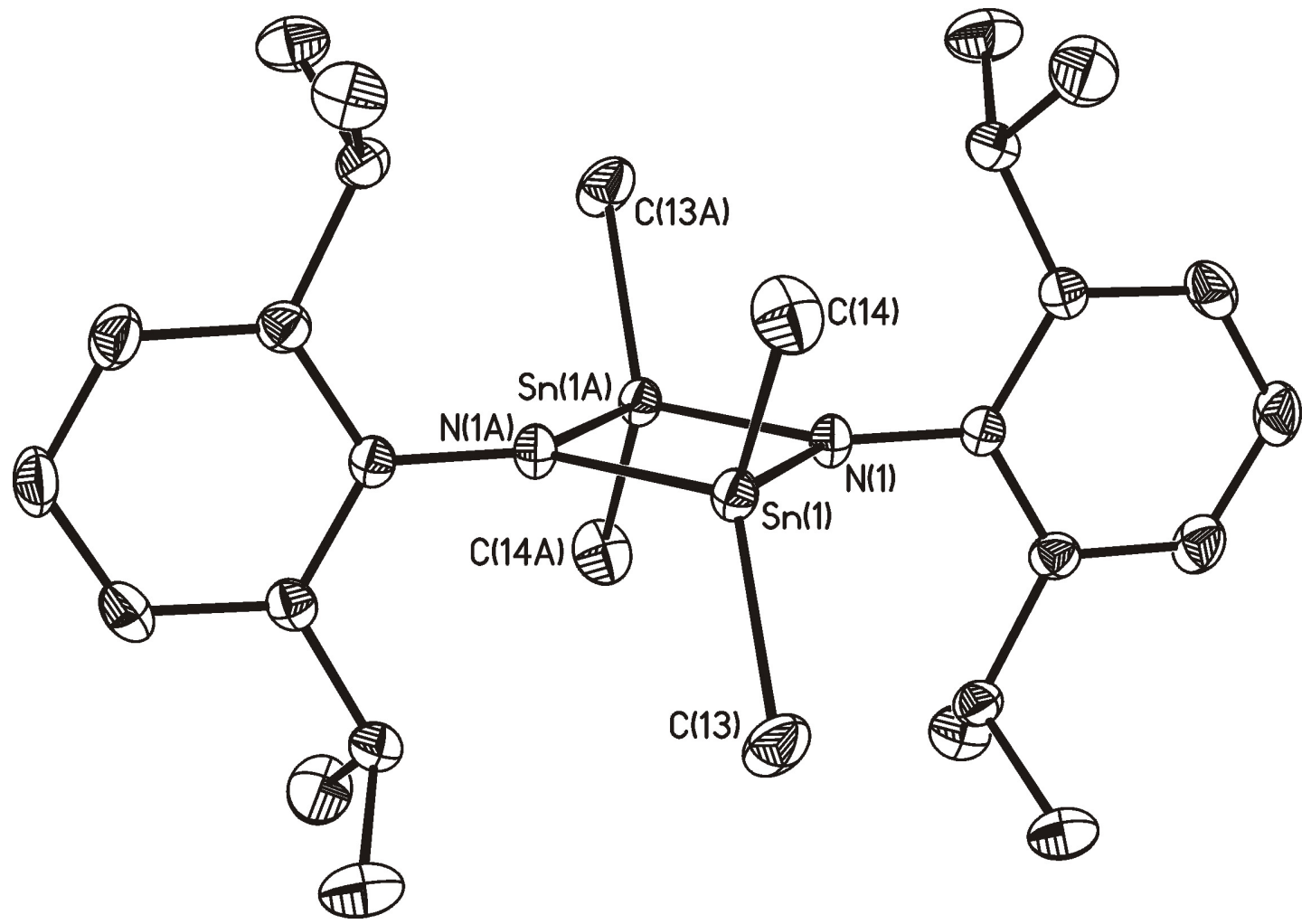

Abb.2. Struktur von 8 im Kristall

Tab.2. Ausgewählte Bindungslängen $[\AA]$ und -winkel $\left[{ }^{\circ}\right]$ von 8

\begin{tabular}{llll}
\hline $\operatorname{Sn}(1)-\mathrm{N}(1)$ & $2.059(2)$ & $\mathrm{N}(1)-\operatorname{Sn}(1)-\mathrm{N}(1 \mathrm{~A})$ & $81.75(9)$ \\
$\operatorname{Sn}(1)-\mathrm{N}(1 \mathrm{~A})$ & $2.047(2)$ & $\mathrm{N}(1)-\operatorname{Sn}(1)-\mathrm{C}(13)$ & $112.93(11)$ \\
$\operatorname{Sn}(1)-\mathrm{C}(13)$ & $2.139(3)$ & $\mathrm{N}(1 \mathrm{a})-\operatorname{Sn}(1)-\mathrm{C}(13)$ & $116.27(11)$ \\
$\operatorname{Sn}(1)-\mathrm{C}(14)$ & $2.137(3)$ & $\mathrm{N}(1)-\operatorname{Sn}(1)-\mathrm{C}(14)$ & $114.15(10)$ \\
& & $\mathrm{N}(1 \mathrm{~A})-\mathrm{Sn}(1)-\mathrm{C}(14)$ & $114.34(11)$ \\
\hline
\end{tabular}




\section{$\mathrm{C}(14)-\operatorname{Sn}(1)-\mathrm{C}(13) \quad 113.66(12)$ \\ $\operatorname{Sn}(1)-\mathrm{N}(1)-\operatorname{Sn}(1 \mathrm{~A}) \quad 98.25(9)$}

8 kristallisiert monoklin in der Raumgruppe $P 2(1) / n$ mit zwei unabhängigen Formeleinheiten pro Elementarzelle. Der Kern von 8 ist ein vieratomiger $\operatorname{Sn}_{2} \mathrm{~N}_{2}$-Ring. Die Zinnatome sind über zwei Stickstoffatome verbrückt. Zur Vervollständigung der Koordinationssphäre trägt jedes Zinnatom zwei Methyl-Gruppen, die Stickstoffatome sind jeweils an einem Ar-Rest gebunden. Die Zinnatome sind leicht verzerrt tetraedrisch koordiniert mit Bindungswinkeln zwischen 81.75 und $116.2^{\circ}$. Der N-Sn-N Bindungswinkel ist mit $81.75^{\circ}$ kleiner als der Sn-N-Sn Bindungswinkel mit 98.25 $5^{\circ}$ Die Zinn-Stickstoff Bindungslängen liegen zwischen 2.047 und $2.059 \AA$ und sind vergleichbar mit denen, die in $\left[t-\mathrm{Bu}_{2} \mathrm{Sn}-\mathrm{N} t-\mathrm{Bu}\right]_{2}(2.059$ und $2.055 \AA)$ und $[t$ $\mathrm{Bu}_{2} \mathrm{SnNSO}_{2} \mathrm{Me}_{2}\left(2.085\right.$ und $2.092 \AA$ ) gefunden wurden. ${ }^{[97]}$

Ein möglicher Mechanismus für die Bildung von $\mathbf{8}$ ist, dass bei der Bildung von 7 ein solches Molekül mit einem weiteren $\mathrm{Me}_{3} \mathrm{SnF}-$ Molekül reagiert, wodurch ein Difluoroaluminium-Anion und ein $\mathrm{Me}_{3} \mathrm{Sn}^{+}$-Kation gebildet werden. Solche Kationen konnten bereits in Lösung durch Licht-induzierten Elektronen-Transfer beobachtet werden. ${ }^{[98]}$ Anschließend bildet das Zinnatom eine Bindung zu einem Stickstoffatom aus. Nach Eliminierung einer Methylgruppe bildet sich das Monomer von 8, wie im Falle von $\left[\left(\mathrm{F}_{3} \mathrm{C}\right)_{3} \mathrm{C}_{6} \mathrm{H}_{2}\right] \mathrm{SnNMes}_{2}{ }^{[99]}$ dimerisiert das Stannaimin zu 8.

\subsection{Versuch zur Umsetzung von 1 mit $n-B_{4} \mathbf{N}\left(H_{2}\right)$}

Ein anderes Fluorierungsreagenz ist $n-\mathrm{Bu}_{4} \mathrm{~N}\left(\mathrm{HF}_{2}\right)$. Wie in unserem Arbeitskreis gezeigt werden konnte, sind Difluordiorganoaluminate durch einfache Umsetzung von $\left[\left(\mathrm{Me}_{3} \mathrm{Si}\right)_{3} \mathrm{CAlMe}_{2}\right]_{2}$ oder $\mathrm{R}_{3} \mathrm{E}(\mathrm{R}=\mathrm{Me}, \mathrm{E}=\mathrm{Al}, \mathrm{Ga}, \mathrm{In})$ mit $n-\mathrm{Bu}_{4} \mathrm{~N}\left(\mathrm{HF}_{2}\right)$ erhältlich. ${ }^{[100]}$ Solche Verbindungen sind seit 1955 bekannt, ${ }^{[4,15,101,102]}$ jedoch waren nur wenige Beispiele $^{[12,103,104]}$ strukturell untersucht worden. Durch Umsetzung von $n$ - $\mathrm{Bu} 4 \mathrm{~N}_{(}\left(\mathrm{HF}_{2}\right)$ mit $\left(\mathrm{RPO}_{3} \mathrm{AlMe}\right)_{4}(\mathrm{R}=\mathrm{Ph}, t$-Bu$)$ sind außerdem fluorhaltige Aluminophosphonate darstellbar. ${ }^{[105]}$ 
Es sollte nun überprüft werden, ob 1 geeignet zur Darstellung von Difluoroorganoaluminaten ist. (Schema 10.) Das angegebene Beispiel bezieht sich auf den Fall einer 1:1 Umsetzung) Die dabei eingesetzten Stöchiometrien von 1 zu $n$ $\mathrm{Bu}_{4} \mathrm{~N}\left(\mathrm{HF}_{2}\right)$ variierten von $1: 1$ bis $1: 3$.

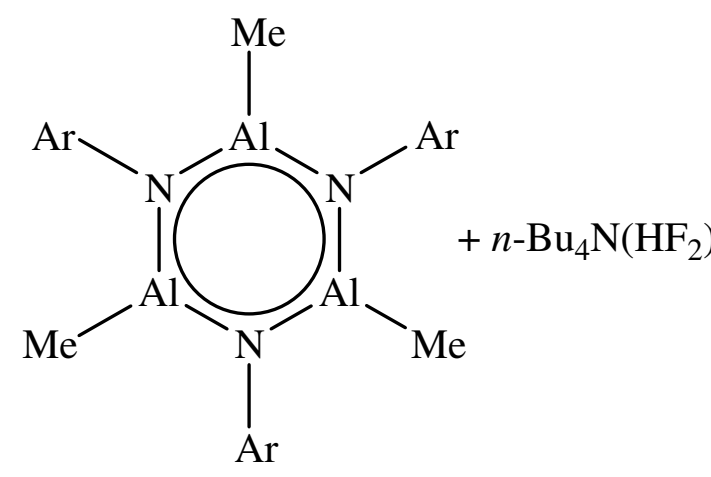

(1)

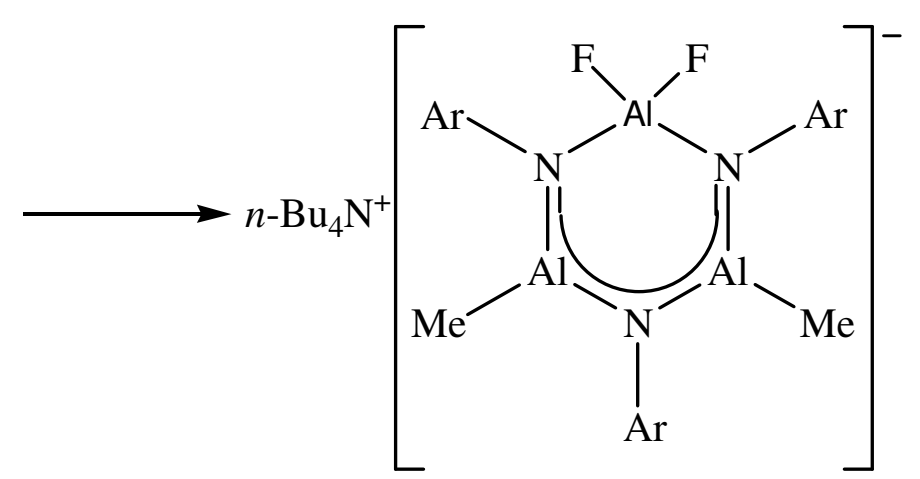

Schema 10.

$\mathrm{Zu} 1$ in THF wurde bei Raumtemperatur eine Lösung von $n-\mathrm{Bu}_{4} \mathrm{~N}\left(\mathrm{HF}_{2}\right)$ in THF tropfenweise zugegeben. Nach Aufarbeitung und unterschiedlichen Kristallisationszeiten wurden nicht die erwarteten Aluminate, sondern die bereits oben zitierte Verbindung $n$ - $\mathrm{Bu}_{4} \mathrm{~N}\left(\mathrm{Me}_{2} \mathrm{AlF}_{2}\right)$ erhalten. Alle erhaltenen spektroskopischen Daten entsprechen denen der Literatur. ${ }^{[100]}$

\subsection{Umsetzung von 1 mit Borhalogeniden und Boranen}

\subsubsection{Umsetzung von 1 mit $\mathbf{F}_{3} \mathbf{B} \cdot \mathbf{O E t}_{2}$}

Wie bereits in der Einleitung erwähnt, werden fluorhaltige Borverbindungen zur Darstellung von Organoaluminiumfluoriden verwendet. Kürzlich berichteten Uhl et al. über die Reaktion eines Carbalan-Clusters $(\mathrm{AlEt})_{7}\left(\mathrm{C} \equiv \mathrm{CHC}_{6} \mathrm{H}_{5}\right)_{2}\left(\mathrm{CCH}_{2} \mathrm{C}_{6} \mathrm{H}_{5}\right)_{3} \mathrm{H}$ mit $\mathrm{HBF}_{4} \cdot \mathrm{OEt}_{2}$, welche zu dem fluorierten Derivat $(\mathrm{AlEt})_{7}\left(\mathrm{C} \equiv \mathrm{CHC}_{6} \mathrm{H}_{5}\right)_{2}\left(\mathrm{CCH}_{2} \mathrm{C}_{6} \mathrm{H}_{5}\right)_{3}\left(\mu_{3}-\mathrm{F}\right)$ 
führte. ${ }^{[106]}$ In diesem Versuch sollte $\mathrm{F}_{3} \mathrm{~B} \cdot \mathrm{OEt}_{2}$ verwendet werden, da es einfacher zu handhaben ist als $\mathrm{F}_{4} \mathrm{BH} \cdot \mathrm{OEt}_{2}$.

$\mathrm{Zu}$ diesem Zweck wurde zu 1 in THF eine Lösung von $\mathrm{F}_{3} \mathrm{~B} \cdot \mathrm{OEt}_{2}$ in $\mathrm{Et}_{2} \mathrm{O}$ tropfenweise gegeben und anschließend 12 Stunden lang bei gleicher Temperatur gerührt. Nach Aufarbeitung, Aufnahme des Rohproduktes mit $n$-Hexan kristallisierte 7 bei $-24{ }^{\circ} \mathrm{C}$ als farbloser Feststoff aus.

\subsubsection{Umsetzung von 1 mit $\mathrm{Cl}_{3} \mathrm{~B}$, Darstellung von $\left[\mathrm{ClAlN}\left(2,6-i-\mathrm{Pr}_{2} \mathrm{C}_{6} \mathrm{H}_{3}\right)\right]_{3} \cdot 3 \mathrm{THF}$} (9)

$\left(\mathrm{Me}_{3} \mathrm{Si}\right)_{3} \mathrm{CAlCl}_{2} \cdot \mathrm{THF}$ konnte durch Reaktion des Dimethylalans mit $\mathrm{Me}_{3} \mathrm{SnCl}$ dargestellt werden. ${ }^{[23]}$ Eine andere Möglichkeit besteht darin, ein Lithiumorganyl mit $\mathrm{Cl}_{3} \mathrm{Al}$ umzusetzen. ${ }^{[107,108]}$ Vor kurzem wurde $\mathrm{Cl}_{3} \mathrm{~B}$ zur Funktionalisierung von $\left[(\mathrm{AlH})_{6}\left(\mathrm{AlNMe}_{3}\right)_{2}\left(\mathrm{CCH}_{2} \mathrm{R}\right)_{6}\right]\left(\mathrm{R}=\mathrm{Ph}, \mathrm{CH}_{2} \mathrm{SiMe}_{3}\right)$ verwendet. $^{[109]}$ Der Vorteil der Reaktion liegt darin, dass überschüssiges $\mathrm{Cl}_{3} \mathrm{~B}$ im Vakuum leicht zu entfernen ist. Schema 11.

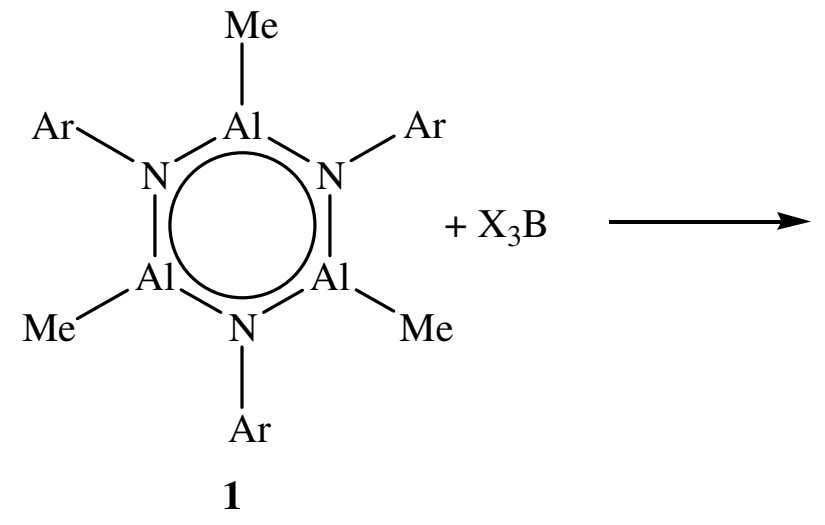

Schema 11.

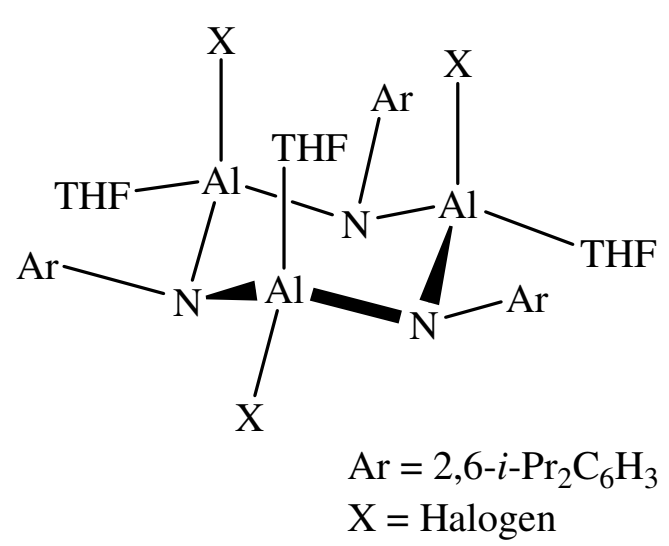

$\mathrm{Ar}=2,6-i-\mathrm{Pr}_{2} \mathrm{C}_{6} \mathrm{H}_{3}$ $\mathrm{X}=$ Halogen

$\mathrm{Zu} 1$ in THF wurde bei $0{ }^{\circ} \mathrm{C}$ eine $\mathrm{Cl}_{3} \mathrm{~B}$ Lösung in $n$-Hexan zugegeben. Die Reaktionslösung wurde anschließend weitere 12 Stunden lang bei Raumtemperatur gerührt. Nach Entfernen des Lösungsmittels im Vakuum wurde das Rohprodukt in 
THF/n-Hexan (1:1) aufgenommen. Die Kristallisation erfolgte bei $-26{ }^{\circ} \mathrm{C}$ über einen Zeitraum von 36 Stunden. 9 kristallisierte als farbloser Feststoff, der bei $229{ }^{\circ} \mathrm{C}$ schmilzt.

Im ${ }^{1} \mathrm{H}-\mathrm{NMR}$ Spektrum in $\mathrm{C}_{6} \mathrm{D}_{6}$ sind die Resonanzen für die Methylgruppen am Aluminium verschwunden. Wie bereits bei $\mathbf{7}$ beobachtet, werden für die Methylprotonen der Isopropylgruppe verschiedene Resonanzen gefunden. Ferner lässt das Spektrum vermuten, dass drei THF Moleküle an den Aluminiumatomen koordiniert sind.

Im EI-Massenspektrum wird bei m/z 726 ein Peak detektiert, der dem Molekülion von 9 abzüglich einer DippN- und einer $i$-Propylgruppe zugeordnet werden kann. Die korrekte Elementaranalyse bestätigt die Zusammensetzung von 9.

Die Struktur von 9 weist starke Fehlordnungen auf, hervorgerufen durch die Isopropylgruppen, deshalb wird auf eine Abbildung der Struktur verzichtet. Sie zeigt jedoch eine zu 7 analoge Bootkonformation mit an den Aluminiumatomen gebundenen THF-Molekülen.

\subsubsection{Umsetzung von 1 mit $\mathrm{Br}_{3} \mathrm{~B}$, Darstellung von $\left[\mathrm{BrAIN}\left(2,6-i-\mathrm{Pr}_{2} \mathrm{C}_{6} \mathrm{H}_{3}\right)\right]_{3} \cdot 3 \mathrm{THF}$} (10)

Wie aus Schema 11 ersichtlich, wurde 1 durch eine $1.0 \mathrm{M} \mathrm{Br}_{3} \mathrm{~B}$ Lösung in $n$ Hexan in das Tribromid 10 überführt. Aus $n$-Hexan kristallisiert 10 bei $-4{ }^{\circ} \mathrm{C}$ als gelber Feststoff aus. 10 hat einen ähnlich hohen Schmelzpunkt wie 7 und 9. Im ${ }^{1} \mathrm{H}-\mathrm{NMR}$ sind die Resonanzen der Aluminium-gebundenen Methylprotonen verschwunden. An den Aluminiumatomen sind wie bei 7 und 9 zusätzlich THF-Moleküle koordiniert. Im EIMassenspektrum findet sich bei m/z 990 der Molekülionenpeak abzüglich eines THFMoleküls. 


\subsubsection{Versuch zur Umsetzung von 1 mit $I_{3} B$}

Die Reaktion von Dimethylalanen mit elementarem Iod führt $\mathrm{zu}$ den entsprechenden Iod-Derivaten. So konnte auf diese Weise $\left[\left(\mathrm{Me}_{3} \mathrm{Si}\right)_{3} \mathrm{CAlMe}_{2} \cdot \mathrm{THF}\right]^{[23]}$ bzw. $\left[\left(3,5-t-\mathrm{Bu}_{2} \mathrm{pz}\right)_{2}\left(\mathrm{AlMe}_{2}\right)_{2}\right]^{[108]}\left(3,5-t \mathrm{Bu}_{2} \mathrm{pz}=3,5-\mathrm{Di}-t\right.$-butylpyrazolat $)$ in die entsprechenden Iodide überführt werden. Da der Versuch, 1 mit elementarem Iod umzusetzen, nur zu Produktgemischen führte, ${ }^{[110]}$ sollte in diesem Versuch untersucht werden, ob das Iod-Derivat von 1 durch Reaktion mit $I_{3} B$ erhältlich ist. Dazu wurde zu $\mathbf{1}$ in THF bei Raumtemperatur $\mathrm{I}_{3} \mathrm{~B}$ in THF gegeben. Nach Aufarbeitung konnte der erhaltene Feststoff nicht identifiziert werden. Eine Variation der Reaktionstemperatur und Stöchiometrie ergab ähnliche Ergebnisse.

\subsubsection{Reaktion von 1 mit $\mathrm{H}_{3} B \cdot T H F$; Darstellung von $\left[\mathrm{MeAIN}\left(2,6-i-\operatorname{Pr}_{2} \mathrm{C}_{6} \mathrm{H}_{3}\right)\right]_{3} \cdot 3$ THF (11)}

Nachdem die Reaktionen von Bortrihalogeniden mit Ausnahme von $\mathrm{I}_{3} \mathrm{~B}$ zur Bildung der Halogenderivate von 1 führte, sollte untersucht werden, ob mit $\mathrm{H}_{3} \mathrm{~B} \cdot \mathrm{THF}$ das Hydrid von 1 darstellbar ist. Es ist seit langem bekannt, dass Diboran unter Einwirkung von Verbindungen mit Elektronenlücken, z.B. Alkylverbindungen von Elementen der 13. Gruppe, an diese sich anlagert und dann eine Alkylgruppe substituiert. ${ }^{[3]}$ (Schema 12.) Auf diese Weise sind Methylderivate des Borans und entsprechender Hydride der 13. Gruppe zugänglich.

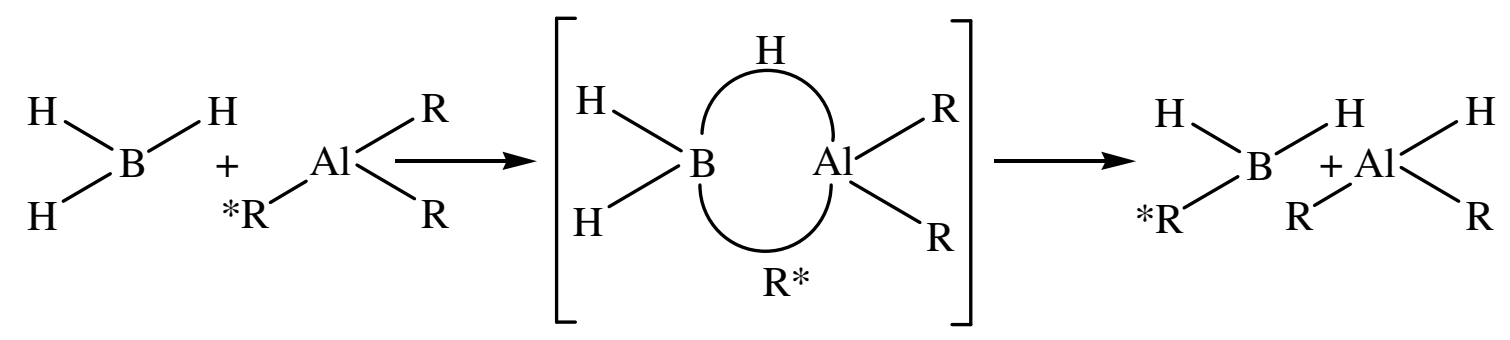

Schema 12. 
Hierzu wurde bei Raumtemperatur zu 1 in THF eine $\mathrm{H}_{3} \mathrm{~B}$ Lösung in THF gegeben. Nach Aufarbeitung erfolgte die Kristallisation, wobei $\mathbf{1 1}$ als farbloser kristalliner Feststoff erhalten werden konnte. Offensichtlich fand kein Austausch der Liganden statt, wie in Schema 12 beschrieben, sondern lediglich THF koordiniert an die Lewis-sauren Aluminiumatome. Da 11 bereits beschrieben wurde, ${ }^{[91]}$ wird auf eine Diskussion der spektroskopischen Daten verzichtet.

\subsection{Versuch zur Umsetzung von 1 mit $\mathrm{PhSi}(\mathrm{C} \equiv \mathrm{CH})_{3}$}

Wie schon mehrfach erwähnt, zeichnet sich 1 durch seine Reaktivität aus, die sowohl koordinative als auch elektronische Ursachen hat. Die Umsetzung mit Fluoriden der 4. Gruppe führte zu adamantanartigen Gerüsten, wobei Fluoratome an die Aluminiumzentren koordinieren und zusätzlich Fluor-Stickstoff-Metathese-Reaktionen unter Bildung neuer bimetallischer Fluoride beobachtet wurden. ${ }^{[89,90]}$ Auch die Umsetzung mit Silantriolen und Triaminosilanen führte zur Bildung von adamantanartigen Gerüsten. ${ }^{[111]}$ Dabei werden jeweils die Protonen der Si-OH-Gruppe bzw. der Si-NH$H_{2}$-Gruppe auf die drei Stickstoffatome des $\mathrm{Al}_{3} \mathrm{~N}_{3}$-Ringes übertragen und es bilden sich Aluminium-Sauerstoffbindungen aus. (Schema 13.) Solche Additionsreaktionen von $\mathrm{HX}, \mathrm{X}_{2}(\mathrm{X}=\mathrm{Hal})$ oder $\mathrm{ROH}$ an polare Bor-StickstoffBindungen wie z.B. Borazin waren seit längerem bekannt. ${ }^{[112]}$

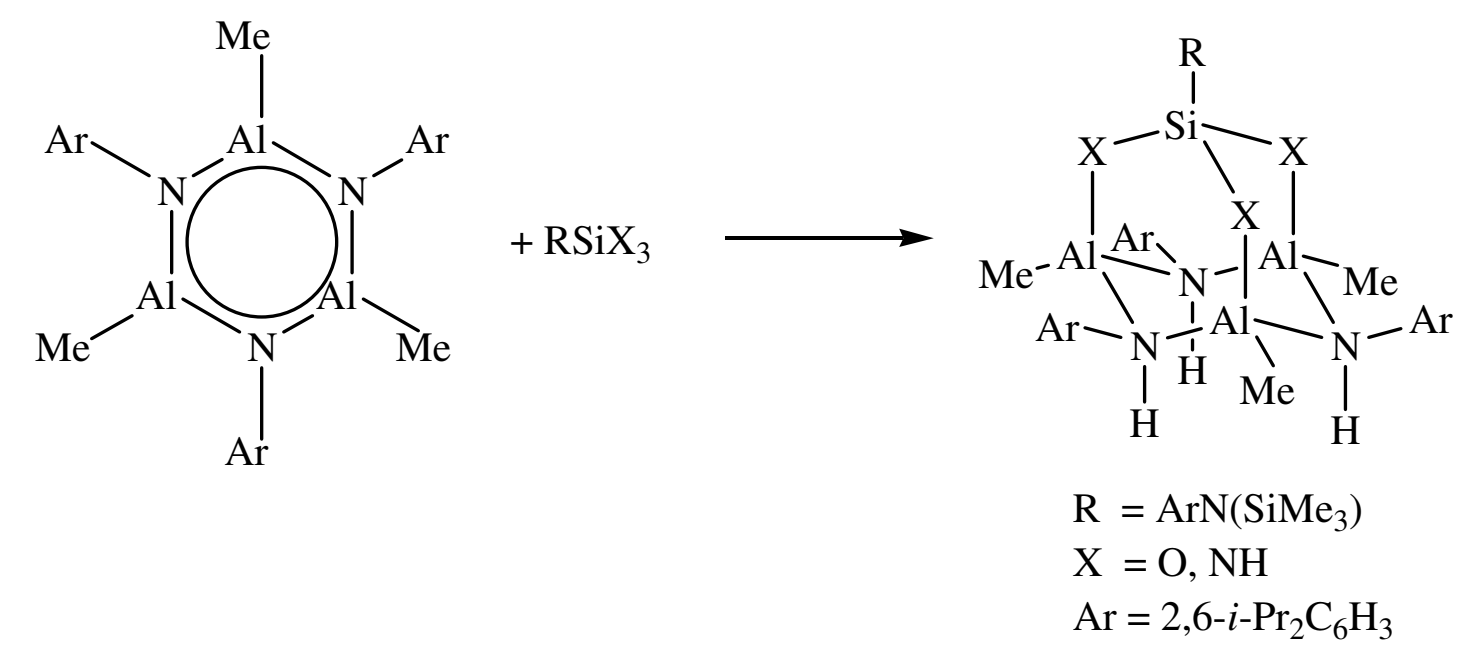

Schema 13. 
Kürzlich veröffentlichten Pinkas et al. ein adamantananalog aufgebautes Aluminiumamidophosphat aus der Reaktion von 1 mit Tris(trimethylsilyl)phosphat. ${ }^{\text {[113] }}$ Ähnlich den Reaktionen mit Silantriolen bilden sich Aluminium-Sauerstoffbindungen aus und der $\mathrm{Al}_{3} \mathrm{~N}_{3}$-Ring bleibt erhalten.

Durch Umsetzung von 1 mit $\mathrm{PhSi}(\mathrm{C} \equiv \mathrm{CH})_{3}{ }^{[114]}$ sollte nun untersucht werden, ob eine Übertragung des Protons $\mathrm{zu}$ den Stickstoffatomen des $\mathrm{Al}_{3} \mathrm{~N}_{3}$-Ringes unter Aluminium-Kohlenstoff-Bindungsbildung erfolgt. Deshalb wurde 1 in Toluol bei -78 ${ }^{\circ} \mathrm{C}$ mit einer Lösung von $\mathrm{PhSi}(\mathrm{C} \equiv \mathrm{CH})_{3}$ in Toluol versetzt. Nach Aufarbeitung wurde das Rohprodukt mit $n$-Pentan gewaschen und filtriert. Der erhaltene braune Feststoff konnte nicht genauer analysiert werden. Da kein Hinweis auf ein Addukt zu finden war, muss davon ausgegangen werden, dass $\mathrm{PhSi}(\mathrm{C} \equiv \mathrm{CH})_{3}$ nicht ausreichend $\mathrm{CH}$-acide ist. Andere Ergebnisse bestätigen diese Annahme. ${ }^{[115]}$

\subsection{Darstellung und Hydrolyse von monoorganylsubstituierten Germaniumhalogeniden}

Im nächsten Kapitel sollten Germanole mit Terphenylliganden synthetisiert werden. Terphenylliganden können eine Vielzahl von ungewöhnlichen Koordinationsgeometrien und Bindungsverhältnisse stabilisieren. ${ }^{[33-37]}$

Wie einleitend erwähnt, sind Organosilanole schon länger bekannt. ${ }^{[64-67]}$ Hingegen sind Verbindungen mit Germanium nur sehr wenig untersucht worden. Ein Grund ist die Tendenz der OH-Gruppen zur Selbstkondensation unter Bildung größerer Aggregate. ${ }^{[74,80]}$ Durch Verwendung von Terphenylen sollten die OH-Gruppen sterisch so abgeschirmt sein, dass die Selbstkondensation verhindert wird.

\subsubsection{Darstellung von 2,6-Bis(26,66-dimethylphenyl)-1-iodbenzol (12)}

Die Darstellung von 2-Iod-m-terphenylen kann über verschiedene Wege erfolgen. Ein Weg ist die „Eintopfreaktion“, ausgehend vom Dichloriodbenzol. (Schema 
14.) In dieser Reaktion wird das Dichloriodbenzol mit der vierfachen Menge des ArMgBr Grignards umgesetzt. Ein Halogen-Metallaustausch wird mittels Iod initiiert und die anschließende zweifache nucleophile Additions-Eliminierungsreaktion über mögliche Arinzwischenstufen ergibt das 2,6-arylsubstituierte Grignard-Reagenz. Dieses wird dann mit elementarem Iod zum Endprodukt überführt. Das erhaltene Rohprodukt 12 wird durch Kristallisation gereinigt. ${ }^{[116]}$<smiles>Clc1cccc(Cl)c1I</smiles>

12

Schema 14.

Eine andere Möglichkeit besteht in der Umsetzung von Dichlorbenzol mit $n$ BuLi bei $-70{ }^{\circ} \mathrm{C}$. Das Lithiumsalz wird anschließend mit zwei Äquivalenten des ArMgBr-Grignards umgesetzt und anschließend mit elementarem Iod gequencht. (Schema 15.) Die Ausbeute entspricht der, wie sie aus der Reaktion mit Dichloriodbenzol erhalten wird. ${ }^{[116,117]}$<smiles>Clc1cccc(Cl)c1</smiles>

Schema 15.

Verbindung 12 wurde über Dichloriodbenzol dargestellt. Bei $-26{ }^{\circ} \mathrm{C}$ kristallisierte 12 als schwachgelber Feststoff. Im ${ }^{1}$ H-NMR Spektrum werden die Resonanzsignale der Methylprotonen bei $\delta 2.04 \mathrm{ppm}$ und für die aromatischen Protonen bei $\delta 6.77$ und 7.08 ppm gefunden. Bei der massenspektrometrischen Untersuchung 
wurde der Molekülionenpeak bei m/z 412 detektiert. Abb. 3 zeigt die Struktur von 12 im Kristall. Ausgewählte Bindungslängen und -winkel sind in Tab. 3 wiedergegeben.

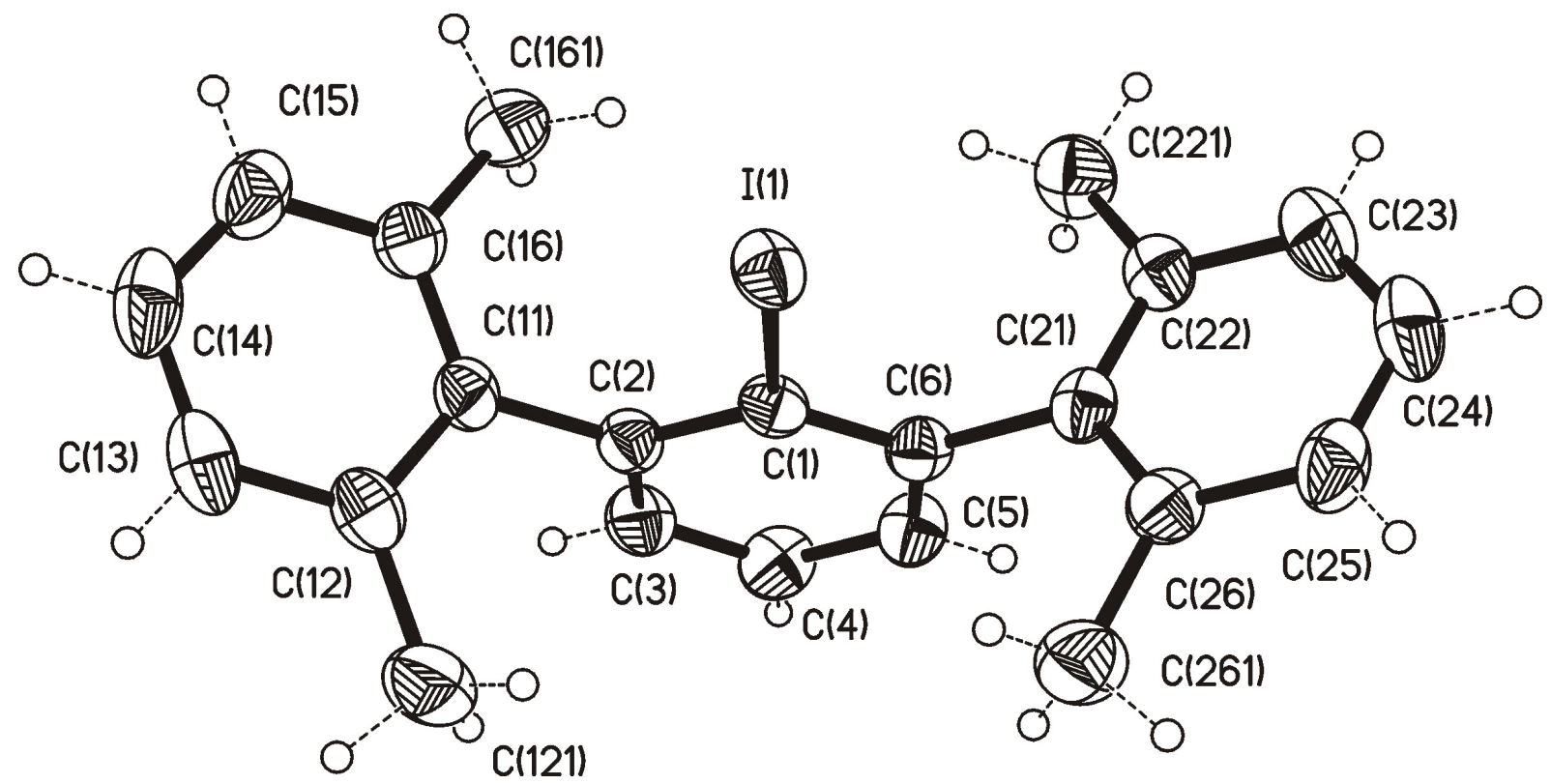

Abb.3. Struktur von 12 im Kristall.

Tab.3. Ausgewählte Bindungslänge $[\AA ̊]$ und -winkel $\left[{ }^{\circ}\right]$ von 12.

\begin{tabular}{ll}
\hline $\mathrm{C}(1)-\mathrm{I}(1)$ & $2.111(3)$ \\
$\mathrm{C}(2)-\mathrm{C}(1)-\mathrm{I}(1)$ & $118.6(2)$ \\
$\mathrm{C}(11)-\mathrm{C}(2)-\mathrm{I}(1)$ & $122.9(2)$ \\
\hline
\end{tabular}

Verbindung 12 kristallisiert orthorhombisch in der Raumgruppe Pbca mit acht unabhängigen Formeleinheiten pro Elementarzelle. Die beiden meta-gebundenen Phenylringe sind in Bezug auf den mittleren Ring aufgrund ihres sterischen Anspruches verdreht. Sämtliche Bindungslängen und -winkel sind vergleichbar mit literaturbekannten Werten. ${ }^{[118,119]}$ 


\subsubsection{Darstellung und Struktur von 2,6-Bis $\left(2^{6}, 6^{6}\right.$-dimethylphenyl)- phenylgermaniumtrichlorid $\left(\mathrm{R}^{*} \mathrm{GeCl}_{3}\right)(13)$}

Durch Zugabe von $n$-BuLi in $n$-Hexan bei $-78{ }^{\circ} \mathrm{C}$ wurde 12 in $n$-Hexan zunächst in das Lithiumsalz überführt, nach Aufarbeitung und erneutem Lösen in $\mathrm{Et}_{2} \mathrm{O}$ wurde bei $-78{ }^{\circ} \mathrm{C} \mathrm{Cl}_{4} \mathrm{Ge}$ zugetropft. (Schema 16.) Nach $18 \mathrm{~h}$ Reaktionszeit und anschließender Filtration wurde das Lösungsmittel im Vakuum entfernt und das Rohprodukt $\mathbf{1 3}$ in THF/n-Hexan (1:1) aufgenommen. Aus dieser Lösung kristallisierten bei Raumtemperatur farblose Kristalle aus, die für eine Einkristall-Röntgenstrukturanalyse geeignet waren.<smiles>[R]c1cccc(Br)c1I</smiles><smiles>[R]c1cccc([R])c1[Al]</smiles><smiles>[R]c1cccc([R])c1C(N)=O</smiles>

$$
\begin{aligned}
& \text { 13: } X=\mathrm{Cl} \\
& \text { 14: } X=\mathrm{Br} \\
& \text { 15: } X=\mathrm{I} \\
& R=2,6-\mathrm{Me}_{2} \mathrm{C}_{6} \mathrm{H}_{3}
\end{aligned}
$$

Schema 16. Allgemeine Darstellungsvorschrift für $\mathrm{R}^{*} \mathrm{GeX}_{3}$

In den ${ }^{1} \mathrm{H}-\mathrm{NMR}$ - und ${ }^{13} \mathrm{C}-\mathrm{NMR}$ Spektren von $\mathbf{1 3}$ sind die Resonanzen für den Terphenylliganden zu erkennen. Der Molekülionenpeak lässt sich im EIMassenspektrum bei m/z 464 identifizieren. Die korrekte Elementaranalyse bestätigt die Zusammensetzung von 13. Abb. 4 zeigt die Struktur von 13 im Kristall. Ausgewählte Bindungslängen und -winkel sind in Tabelle 4 wiedergegeben. 


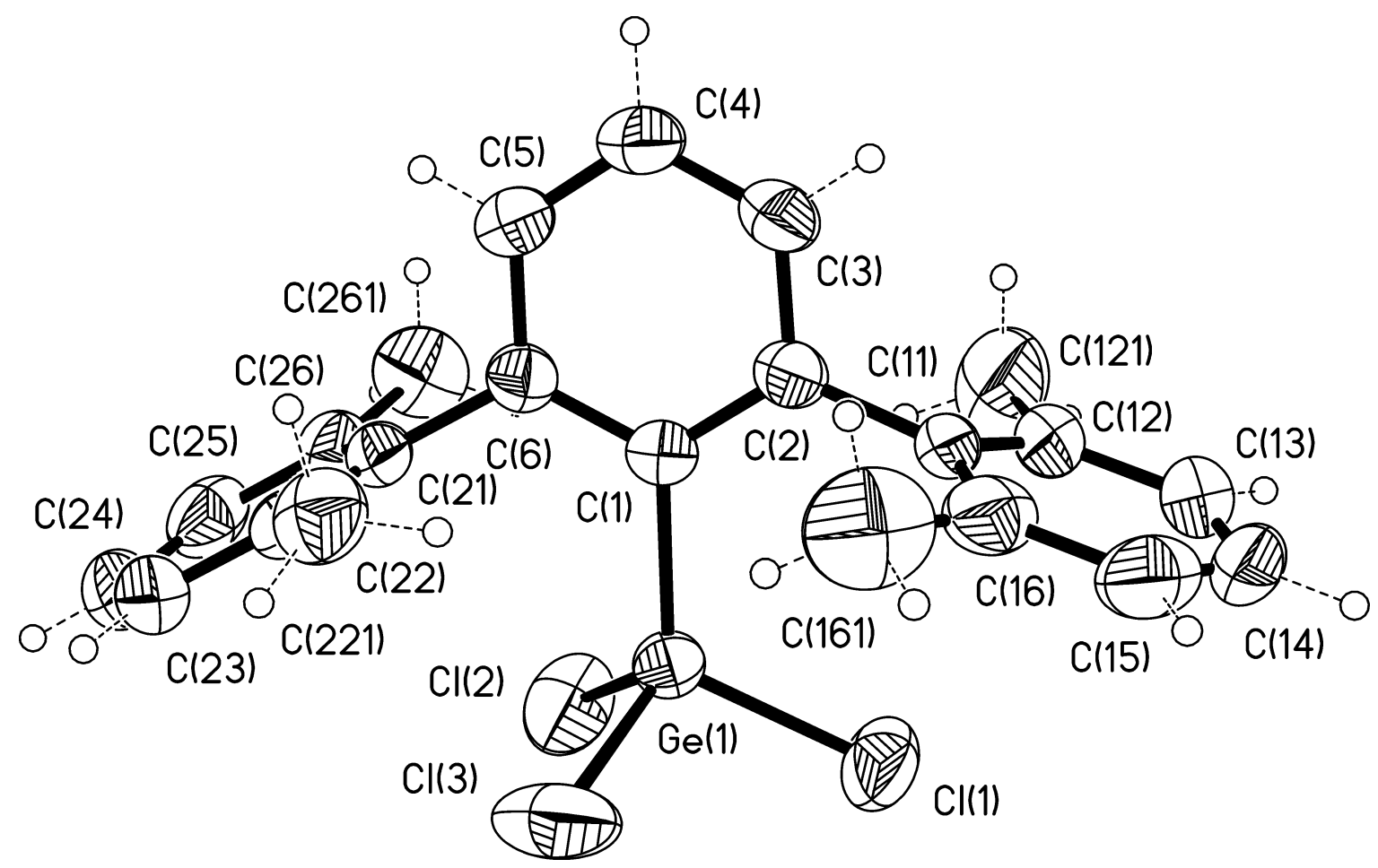

Abb. 4. Abbildung von 13 im Kristall

Tab. 4. Ausgewählte Bindungslängen $[\AA ̊]$ und -winkel $\left[^{\circ}\right]$ von 13

\begin{tabular}{llll}
\hline $\mathrm{Ge}(1)-\mathrm{C}(1)$ & $1.946(4)$ & $\mathrm{C}(1)-\mathrm{Ge}(1)-\mathrm{Cl}(1)$ & $117.91(13)$ \\
$\mathrm{Ge}(1)-\mathrm{Cl}(1)$ & $2.1156(14)$ & $\mathrm{C}(1)-\mathrm{Ge}(1)-\mathrm{Cl}(2)$ & $113.98(13)$ \\
$\mathrm{Ge}(1)-\mathrm{Cl}(2)$ & $2.1269(16)$ & $\mathrm{C}(1)-\mathrm{Ge}(1)-\mathrm{Cl}(3)$ & $113.83(13)$ \\
$\mathrm{Ge}(1)-\mathrm{Cl}(3)$ & $2.1287(16)$ & $\mathrm{Cl}(1)-\mathrm{Ge}(1)-\mathrm{Cl}(2)$ & $102.14(7)$ \\
& & $\mathrm{Cl}(1)-\mathrm{Ge}(1)-\mathrm{Cl}(3)$ & $102.87(7)$ \\
& & $\mathrm{Cl}(2)-\mathrm{Ge}(1)-\mathrm{Cl}(3)$ & $104.42(8)$ \\
\hline
\end{tabular}

Verbindung 13 kristallisiert in der orthorhombischen Raumstruktur Pbca mit acht unabhängigen Formeleinheiten pro Elementarzelle. Zentrale Einheit von $\mathbf{1 3}$ ist ein vierfach koordiniertes Germaniumatom, welches mit drei Chloratomen und dem C(1)Atom des Liganden verbunden ist. Die Germanium-Chlor-Bindungslängen entsprechen 
denen in der Literatur. ${ }^{[120,121]}$ Das Germaniumatom nimmt mit seinen Liganden eine leicht verzerrte, tetraedrische Koordination ein.

\subsubsection{Darstellung und Struktur von $2,6-B i s\left(2^{6}, 6^{6}-\right.$ dimethylphenyl)- phenylgermaniumtribromid $\left(\mathbf{R}^{*} \mathrm{GeBr}_{3}\right)(14)$}

Wie in Kapitel 3.5.2. beschrieben, reagierte 12 erst mit $n$-BuLi in $n$-Hexan und anschließend wurde eine Lösung aus $\mathrm{Br}_{4} \mathrm{Ge}$ in $\mathrm{Et}_{2} \mathrm{O}$ in die etherische Lösung des Lithiumsalzes gegeben. Nach Entfernen des Lösungsmittels im Vakuum und Aufnahme des Rohproduktes in $n$-Hexan kristallisierte bei $0{ }^{\circ} \mathrm{C} \mathbf{1 4}$ in Form quaderförmiger, gelblicher Kristalle aus.

Das ${ }^{1}$ H-NMR Spektrum von 14 zeigt die typischen Resonanzlinien des Terphenylliganden. Im EI-Massenspektrum ist der Molekülionenpeak bei m/z $598 \mathrm{zu}$ finden. Die Elementaranalyse bestätigt die korrekte Zusammensetzung von 14.

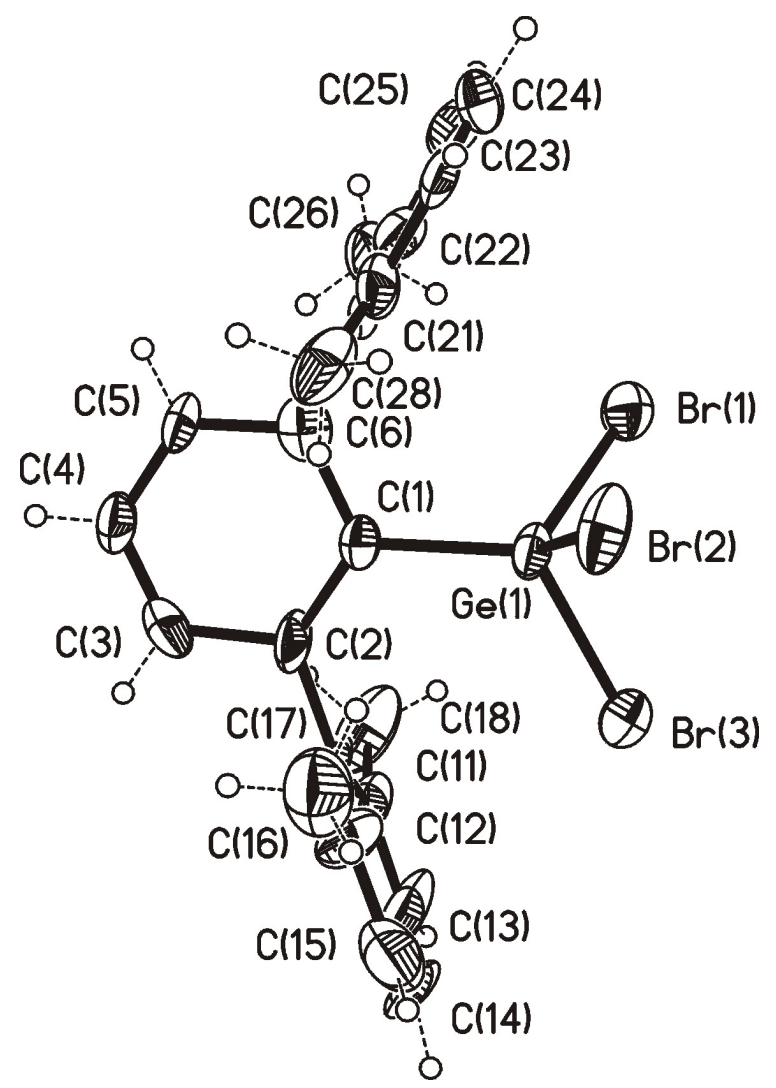

Abb.5. Struktur von $\mathbf{1 4}$ im Kristall 
Tab. 5. Ausgewählte Bindungslängen [̊] und -winkel $\left[{ }^{\circ}\right]$ von 14.

\begin{tabular}{llll}
\hline $\mathrm{Ge}(1)-\mathrm{C}(1)$ & $1.942(14)$ & $\mathrm{C}(1)-\mathrm{Ge}(1)-\operatorname{Br}(1)$ & $113.7(5)$ \\
$\mathrm{Ge}(1)-\operatorname{Br}(1)$ & $2.295(2)$ & $\mathrm{C}(1)-\mathrm{Ge}(1)-\operatorname{Br}(2)$ & $113.0(4)$ \\
$\mathrm{Ge}(1)-\operatorname{Br}(2)$ & $2.292(2)$ & $\mathrm{C}(1)-\mathrm{Ge}(1)-\operatorname{Br}(3)$ & $118.9(5)$ \\
$\mathrm{Ge}(1)-\operatorname{Br}(3)$ & $2.282(2)$ & $\mathrm{Br}(1)-\mathrm{Ge}(1)-\operatorname{Br}(2)$ & $105.60(10)$ \\
& & $\mathrm{Br}(1)-\mathrm{Ge}(1)-\operatorname{Br}(3)$ & $101.68(9)$ \\
& & $\mathrm{Br}(2)-\mathrm{Ge}(1)-\mathrm{Br}(3)$ & $102.32(9)$ \\
\hline
\end{tabular}

14 kristallisiert isomorph zu 13 in der Raumgruppe Pbca. Die Bindungslängen und -winkel sind vergleichbar mit literaturbekannten Werten. ${ }^{[122,123]}$

\subsubsection{Darstellung von $2,6-B i s\left(2^{6}, 6^{6}\right.$-dimethylphenyl)phenylgermaniumtriiodid (15)}

Die Darstellung des Triiodides erfolgte analog der in den vorherigen Kapiteln beschriebenen Vorschrift. Abweichend davon war, dass wegen der schlechten Löslichkeit von $\mathrm{I}_{4} \mathrm{Ge}$ in $\mathrm{Et}_{2} \mathrm{O}$ bzw. THF in Toluol gearbeitet wurde, so dass eine Lösung aus $\mathrm{I}_{4} \mathrm{Ge}$ in Toluol zu dem in Ether gelösten Lithiumsalz gegeben wurde. Aus $n$-Hexan kristallisierte 15 als gelber Feststoff.

15 hat einen Schmelzpunkt von $143{ }^{\circ} \mathrm{C}$, der deutlich niedriger als der bei Verbindungen 13 und 14 ist. Im ${ }^{1} \mathrm{H}-\mathrm{NMR}$ Spektrum ist bei $\delta 2.03 \mathrm{ppm}$ die Resonanz der Methylprotonen des Liganden zu finden. Das EI-Massenspektrum zeigt bei m/z 739 den Molekülionenpeak von 15. 


\subsubsection{Darstellung von $2,6-\operatorname{Bis}\left(2^{6}, 6^{6}\right.$-dimethylphenyl)phenylgermaniumtrifluorid (16)}

Das Einsatzgebiet von $\mathrm{Me}_{3} \mathrm{SnF}$ umfasst nahezu alle Gruppen des Periodensystems. ${ }^{[16-23]}$ Erst vor kurzem gelang uns die Darstellung und strukturelle Charakterisierung von $\left[(\mathrm{Dipp})_{2} \mathrm{NacNacMgF}\right]_{2} \cdot{ }^{[124]}$ Auch Verbindungen der 14. Gruppe mit terminalen Fluoratomen waren auf diesem Wege darstellbar, so berichtete unser Arbeitskreis über die Darstellung von (Dipp) ${ }_{2} \mathrm{NacNacGeF}^{[47]}$

Nachdem sämtliche Halogenderivate mit Ausnahme von Fluor dargestellt werden konnten, sollte nun untersucht werden, ob die Darstellung des Fluorderivates mit $\mathrm{Me}_{3} \mathrm{SnF}$ als Fluorierungsreagenz möglich ist. Schema 15.

$$
\begin{aligned}
& \mathrm{RGeX}_{3}+3 \mathrm{Me}_{3} \mathrm{SnF} \stackrel{-3 \mathrm{Me}_{3} \mathrm{SnX}}{\longrightarrow} \mathrm{RGeF}_{3} \\
& \text { 16: } \mathrm{R}=2,6-\left(\mathrm{Me}_{2} \mathrm{C}_{6} \mathrm{H}_{3}\right)_{2} \mathrm{C}_{6} \mathrm{H}_{3}, \mathrm{X}=\mathrm{Cl} \\
& \text { 17: } \mathrm{R}=2,6-i-\mathrm{Pr}_{2} \mathrm{C}_{6} \mathrm{H}_{3}, \mathrm{X}=\mathrm{Br}
\end{aligned}
$$

Schema 15. Darstellung von $\mathrm{RGeF}_{3}$.

13 und eine äquimolare Menge an $\mathrm{Me}_{3} \mathrm{SnF}$ in Toluol rührten bei Raumtemperatur für 24 Stunden. Nach Aufarbeitung wurde 16 durch fraktionierte Destillation (Sdp.: $120^{\circ} \mathrm{C} / 0.2$ Torr) gereinigt.

Im ${ }^{1}$ H-NMR Spektrum sind die Protonen des Terphenylliganden jeweils bei $\delta$ 2.01 bzw. 6.83 - 7.08 ppm zu finden. Für die Fluoratome ergibt sich im ${ }^{19}$ F-NMR Spektrum eine Verschiebung von $\delta-42.83$ ppm, die vergleichbar der Verschiebung der Fluoratome in $2,4,6-t-\mathrm{Bu}_{3} \mathrm{C}_{6} \mathrm{H}_{2} \mathrm{GeF}_{3}{ }^{[125]}(\delta=-47.5 \mathrm{ppm})$ ist. Der Molekülionenpeak wird im EI-Massenspektrum bei m/z 415 detektiert. 


\subsubsection{Darstellung von $\operatorname{DippN}\left(\mathrm{SiMe}_{3}\right) \mathrm{GeF}_{3}(17)$}

Die nächste Ausgangsverbindung zur Fluorierung war $\operatorname{DippN}\left(\mathrm{SiMe}_{3}\right) \mathrm{GeBr}_{3}$, da dessen Ligand DippNSiMe 3 relativ einfach zu synthetisieren ist und bereits bei der Darstellung von Organoaluminiumfluoride angewendet wurde. ${ }^{[22]}$ Auch konnten durch diesen Liganden kondensationsstabile Triaminosilane und Silantriole ${ }^{[69]}$ isoliert werden.

Verbindung 17 hat einen Siedepunkt von $143{ }^{\circ} \mathrm{C} / 0.2$ Torr. Neben den typischen Resonanzlinien für die Protonen des DippNSiMe 3 -Liganden im ${ }^{1} \mathrm{H}-\mathrm{NMR}$ Spektrum wird eine Resonanz für die Fluoratome im ${ }^{19}$ F-NMR Spektrum bei $\delta-39.79$ ppm bestimmt. Wie bei Verbindung 17 kann der Molekülionenpeak bei m/z 379 detektiert werden.

\subsection{Versuche zur Hydrolyse von Monoorganylgermaniumtrihalogeniden}

Organosilanole repräsentieren eine Klasse von Verbindungen, die seit langem Gegenstand einer Vielzahl von Untersuchungen z. B. als „building blocks“ zur Darstellung von molekularen Metallsiloxanen und verwandter Materialien sind. ${ }^{[64]}$ Stickstoff-gebundene Silantriole sind durch Hydrolyse von $\operatorname{ArN}\left(\mathrm{SiMe}_{3}\right) \mathrm{SiCl}_{3}$ in Anwesenheit von $\mathrm{PhNH}_{2}$ als Base erhältlich. ${ }^{[69]}$ Vor kurzem berichtete unser Arbeitskreis über die Darstellung einer Monoorganozinnsäure, ${ }^{[126]}$ bei deren Synthese ebenfalls $\mathrm{PhNH}_{2}$ als Base zur Anwendung kam.

Im Gegensatz dazu gibt es nur wenige Germanole, hauptsächlich weil sie eine höhere Tendenz zur Eigenkondensation haben. ${ }^{[73-79]}$ In diesem Abschnitt sollten Monoorganylgermaniumtrihalogenide hydrolysiert werden. Da sich die Darstellung des Terphenylliganden als aufwendig erwies, wurde bei den Versuchen hauptsächlich $\operatorname{DippN}\left(\mathrm{SiMe}_{3}\right) \mathrm{GeBr}_{3}$ verwendet. Mit dieser Verbindung wurde die Darstellung aminoverbrückter Germanium-Verbindungen möglich, wo das Germaniumatom zusätzlich terminale $\mathrm{NH}_{2}$-Gruppen besitzt. ${ }^{[127]}$ 


\subsubsection{Umsetzung von DippN(SiMe $) \mathrm{GeBr}_{3}$ mit $\mathrm{PhNH}_{2}$ und $\mathrm{H}_{2} \mathrm{O}$}

In diesem Versuch sollte $\mathrm{DippN}\left(\mathrm{SiMe}_{3}\right) \mathrm{GeBr}_{3}$ in Anwesenheit einer Base hydrolysiert werden. Das bei der Hydrolyse entstehende $\mathrm{HBr}$ wurde mit $\mathrm{PhNH}_{2}$ durch Bildung des Hydrobromids abgefangen.

$\mathrm{Zu}$ DippN $\left(\mathrm{SiMe}_{3}\right) \mathrm{GeBr}_{3}$ in $\mathrm{Et}_{2} \mathrm{O}$ wurde bei $-78{ }^{\circ} \mathrm{C}_{2} \mathrm{O}$ und Anilin in $\mathrm{Et}_{2} \mathrm{O}$ gegeben, dabei bildete sich ein farbloser Niederschlag aus $\mathrm{PhNH}_{2} \cdot \mathrm{HBr}$. Nachdem die Reaktionslösung 24 Stunden bei Raumtemperatur rührte, wurde der Niederschlag abgetrennt und das Lösungsmittel im Vakuum entfernt. Es zeigte sich, dass eventuell entstandenes Produkt mit Bildung des Anilin-Salzes ausgefallen war. Versuche die Verbindungen voneinander zu trennen schlugen fehl.

\subsubsection{Umsetzung von DippN( $\left(\mathrm{SiMe}_{3}\right) \mathrm{GeBr}_{3}$ mit Pyridin und $\mathrm{H}_{2} \mathrm{O}$; Darstellung von $\left[\left(\operatorname{DippN}\left(\mathrm{SiMe}_{3}\right) \mathrm{Ge}_{4} \mathrm{O}_{6}\right](18)\right.$}

Durch den Einsatz einer anderen Base sollte das unter 3.6.1. beschriebene Trennungsproblem verhindert werden. Bei diesem Versuch diente Pyridin als $\mathrm{HBr}$ Fänger, sonst wurde analog gearbeitet. Nach Aufarbeitung und Aufnahme in $n$-Hexan kristallisierte nach 36 Stunden bei $-26{ }^{\circ} \mathrm{C}$ 18, das Kondensationsprodukt des Germantriols $\left(\mathrm{RGe}(\mathrm{OH})_{3}\right)$, in Form quaderförmiger Kristalle aus. (Schema 16.).

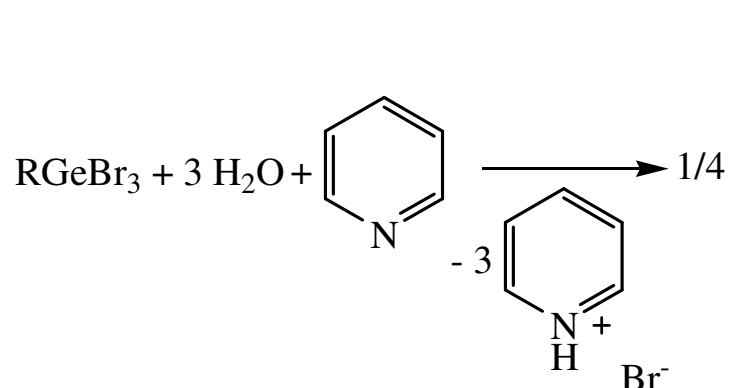

$\mathrm{Br}^{-}$<smiles>[R]C1([R])OC2([R])OC([R])(O2)C([R])(O)O1</smiles>

18

$$
\mathrm{R}=2,6-i-\mathrm{Pr}_{2} \mathrm{C}_{6} \mathrm{H}_{3} \mathrm{~N}\left(\mathrm{SiMe}_{3}\right)
$$

Schema 16. 
18 hat einen Schmelzbereich von $214-218{ }^{\circ} \mathrm{C}$. Im ${ }^{1} \mathrm{H}-\mathrm{NMR}$ Spektrum sind nur die Resonanzlinien der Protonen des Liganden und keine Resonanzen für $\mathrm{OH}-\mathrm{Gruppen}$ zu finden. Im EI-Massenspektrum wird bei m/z 1087 der Molekülionenpeak abzüglich einer DippN($\left(\mathrm{SiMe}_{3}\right)$ - und drei Methylgruppen gemessen. Allerdings wird im IRSpektrum eine Bande bei $\widetilde{v}=3616 \mathrm{~cm}^{-1}$ detektiert. Diese Bande ist einer OH-Gruppe zuzuordnen. ${ }^{[81]}$ Vermutlich ist Verbindung 18 nicht einheitlich, wie in Schema 16 gezeigt, sondern eine Mischung von Hydrolyse-Produkten.

\subsubsection{Umsetzung von $\operatorname{DippN}\left(\mathrm{SiMe}_{3}\right) \mathrm{GeBr}_{3} \mathrm{mit}^{\mathrm{H}_{2}} \mathrm{O}_{2}$}

Da alle Versuche, durch Umsetzung von Halogengermanen in Anwesenheit von Basen zu Germanolen zu kommen, lediglich zu Produktmischungen führten, sollte ein anderer Weg beschritten werden. Im Arbeitskreis gelang die Überführung eines Tetrachlordisilanes in die entsprechende Hydroxy-Verbindung durch Umsetzung mit $\mathrm{H}_{2} \mathrm{O}_{2}$ in Toluol. ${ }^{[128]}$ Analog wurde deshalb zu DippN $\left(\mathrm{SiMe}_{3}\right) \mathrm{GeBr}_{3}$ in Toluol bei Raumtemperatur eine 15 \%ige $\mathrm{H}_{2} \mathrm{O}_{2}$ Lösung gegeben. Nach Aufarbeitung konnte nur quantitativ das Ausgangsprodukt isoliert werden.

\subsubsection{Umsetzung von $\left(\operatorname{DippN}\left(\mathrm{SiMe}_{3}\right) \mathrm{Ge}\left(\mathrm{NH}_{2}\right)_{2}\right)_{2} \mathrm{NH}$ und $\mathrm{H}_{2} \mathrm{O}$}

Die Darstellung des Tetrahydroxydisilans $\left(\mathrm{BisSi}(\mathrm{OH})_{2} \mathrm{Si}(\mathrm{OH})_{2} \mathrm{Bis} ; \quad \mathrm{Bis}=\right.$ Bistrimethylsilylmethyl) war mittels $\mathrm{H}_{2} \mathrm{O}_{2}$ möglich. Eine andere Möglichkeit das Tetrahydroxydisilan $\mathrm{zu}$ erhalten, bestand darin, das Tetraaminodisilan mit $\mathrm{H}_{2} \mathrm{O}$ umzusetzen. ${ }^{[128,129]}$

Da DippN( $\left.\mathrm{SiMe}_{3}\right) \mathrm{Ge}\left(\mathrm{NH}_{2}\right)_{3}$ bisher nicht dargestellt und nur das über eine NHGruppe verbrückte $\left(\mathrm{DippN}\left(\mathrm{SiMe}_{3}\right) \mathrm{Ge}\left(\mathrm{NH}_{2}\right)_{2}\right)_{2} \mathrm{NH}$ literaturbekannt ist, ${ }^{[127]}$ wurde $\mathrm{zu}$ diesem in $\mathrm{Et}_{2} \mathrm{O}$ bei $-78{ }^{\circ} \mathrm{C} \mathrm{H}_{2} \mathrm{O}$ tropfenweise zugegeben. Nach Aufarbeitung kristallisierte 18 bei $0{ }^{\circ} \mathrm{C}$ als Hauptprodukt. 


\subsubsection{Umsetzung von $\operatorname{DippN}\left(\mathrm{SiMe}_{3}\right) \mathrm{GeBr}_{3}$ mit $\mathrm{KOH}$ im Zwei-Phasensystem Ammoniak/Toluol}

Eine Alternative $\mathrm{zu}$ den Umsetzungen Organohalogengermaniumverbindungen bzw. Aminogermanen mit $\mathrm{H}_{2} \mathrm{O}$ stellt die Reaktion mit KOH im Zwei-Phasensystem Toluol/Ammoniak dar. Durch solch eine Reaktion konnten vor kurzem (Dipp) $)_{2} \mathrm{NacNacAl}(\mathrm{OH})_{2}{ }^{[130]}$ und ein Organozirconiumoxid synthetisiert ${ }^{[131]}$ werden.

Über Na getrockneter Ammoniak kondensierte bei $-78{ }^{\circ} \mathrm{C}$ zu einer Suspension aus DippN( $\left.\mathrm{SiMe}_{3}\right) \mathrm{GeBr}_{3}$ und $\mathrm{KOH}$ in Toluol ein. Anschließend rührte das System 30 Minuten lang bei dieser Temperatur, danach wurde unter ständigem Rühren die Temperatur schrittweise auf Raumtemperatur gebracht, wobei überschüssiger Ammoniak verdampfte. Nach Entfernen von $\mathrm{KCl}$ wurde das Lösungsmittel im Vakuum abgezogen. Es wurde ein farbloser Feststoff erhalten, der jedoch nicht als einheitliches Produkt vorliegt.

Im ${ }^{1} \mathrm{H}-\mathrm{NMR}$ zeigen sich bei $\delta 2.00,2.25$ und 3.18 ppm breite Singuletts, die sich $\mathrm{OH}-$ Gruppen zuordnen lassen. Im IR-Spektrum ist eine OH-Bande bei $\tilde{v}=3614 \mathrm{~cm}^{-1}$ zu finden. ${ }^{[81]}$ Im EI-Massenspektrum wird der Molekülionenpeak u.a. von 18 detektiert.

\subsubsection{Umsetzung von 2,6-Bis(26,6'-dimethylphenyl)phenylgermaniumtrichlorid mit $\mathrm{KOH} / \mathrm{NH}_{3}$; Darstellung von $\left[\left(\mathrm{R}^{*} \mathrm{Ge}\right)_{4} \mathrm{O}_{6}\right](19)$}

Da der sterische Anspruch des DippN( $\left.\mathrm{SiMe}_{3}\right)$-Liganden nicht ausreichte, um kondensationsstabile Germanole $\mathrm{zu}$ erhalten, sollte mit dem Terphenyl-Liganden gearbeitet werden. Die Durchführung des Experimentes erfolgte analog Kapitel 3.6.5.

Das Massenspektrum indiziert, dass 19 als adamantanartige Verbindung vorliegt. Auch die Erhöhung des sterischen Anspruches hatte keine Konsequenzen für den Erhalt von terminalen OH-Gruppen. Im IR-Spektrum finden sich Banden bei $\tilde{v}=$ $3618 \mathrm{~cm}^{-1}$, die sich OH-Gruppen zuordnen lassen. ${ }^{[81]} \mathrm{Im}{ }^{1} \mathrm{H}-\mathrm{NMR}$ Spektrum sind keine $\mathrm{OH}-$ Protonen zu finden. 


\subsection{Umsetzungen von Verbindungen mit niedervalenten Elementen der 14. Gruppe}

\subsubsection{Darstellung und Struktur von (Dipp) ${ }_{2} \mathrm{NacNacAlHBH}_{2}(20)$}

Vor kurzem konnte ein seltenes Germanium(II)hydrid aus der Reaktion von (Dipp) ${ }_{2} \mathrm{NacNacGeCl}$ und $\mathrm{NaBH}_{4}$ dargestellt werden. ${ }^{[47]}$ Dabei bildet das Germaniumatom eine Bindung zu einem Wasserstoff- und zwei Bindungen zu den Stickstoffatomen des (Dipp) ${ }_{2} \mathrm{NacNac}-L i g a n d e n$. Zusätzlich ist eine $\mathrm{BH}_{3}$-Gruppe über das freie Elektronenpaar des Germaniums(II) gebunden. Diese Verbindung sollte bezüglich ihrer Reaktivität und dem Reduktionspotential des Germaniums gegenüber $\mathrm{H}_{3} \mathrm{Al} \cdot \mathrm{NMe}_{3}$ untersucht werden. (Schema 19.) Hierbei sollte untersucht werden, ob gegebenenfalls eine Reduktion des Aluminiums und/oder des Bors zu beobachten ist

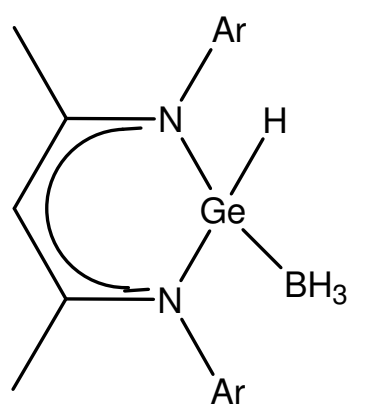

$\mathrm{Ar}=2,6-i-\mathrm{Pr}_{2} \mathrm{C}_{6} \mathrm{H}_{3}$
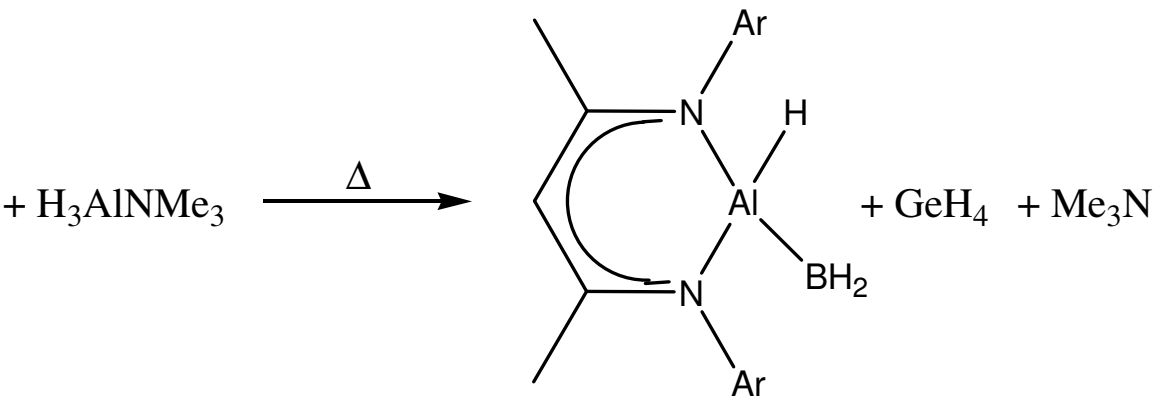

20

Schema 19.

$\mathrm{Zu}$ (Dipp) NacNacGeH$\cdot \mathrm{BH}_{3}$ in Toluol wurde bei Raumtemperatur eine Lösung aus $\mathrm{H}_{3} \mathrm{Al} \cdot \mathrm{NMe}_{3}$ in Toluol gegeben. Nachdem die Reaktionslösung drei $\mathrm{h}$ refluxierte, wurden alle flüchtigen Bestandteile im Vakuum entfernt. Der Rückstand wurde dann mit $10 \mathrm{ml} n$-Hexan aufgenommen. Bei $-26{ }^{\circ} \mathrm{C}$ kristallisierte 20 in Form schwach gelber Kristalle aus.

Bei den massenspektrometrischen Untersuchungen konnte ein Peak bei m/z 458 gefunden werden, der dem Molekülionenpeak von 20 zuzuordnen ist. Das Signal der 
höchsten Intensität bei $\mathrm{m} / \mathrm{z} 445$ entspricht dem Molekülionenpeak abzüglich der $\mathrm{BH}_{2}$ Gruppe. Im ${ }^{1} \mathrm{H}-\mathrm{NMR}$ Spektrum sind die Resonanzen für den NacNac-Liganden zu finden. Die Verschiebung für das Signal des Boratoms wird im ${ }^{11}$ B-NMR Spektrum bei $\delta$-7.53 ppm detektiert und ist, verglichen mit der der Ausgangsverbindung ( $\delta$-43 ppm), ${ }^{[47]}$ ins Tieffeld verschoben. Im IR-Spektrum sind die Banden der BH- bzw. AlHGruppen bei $\tilde{v}=2500$ bzw. $1832 \mathrm{~cm}^{-1}$ zu finden und liegen in Literatur-bekannten Bereichen $\left(\tilde{v}=2500-2600\right.$ bzw. 1800-1900). ${ }^{[132-134]}$

Abb. 6 zeigt die Struktur von 20 im Kristall. Ausgewählte Bindungslängen und winkel sind in Tab. 6 wiedergegeben.

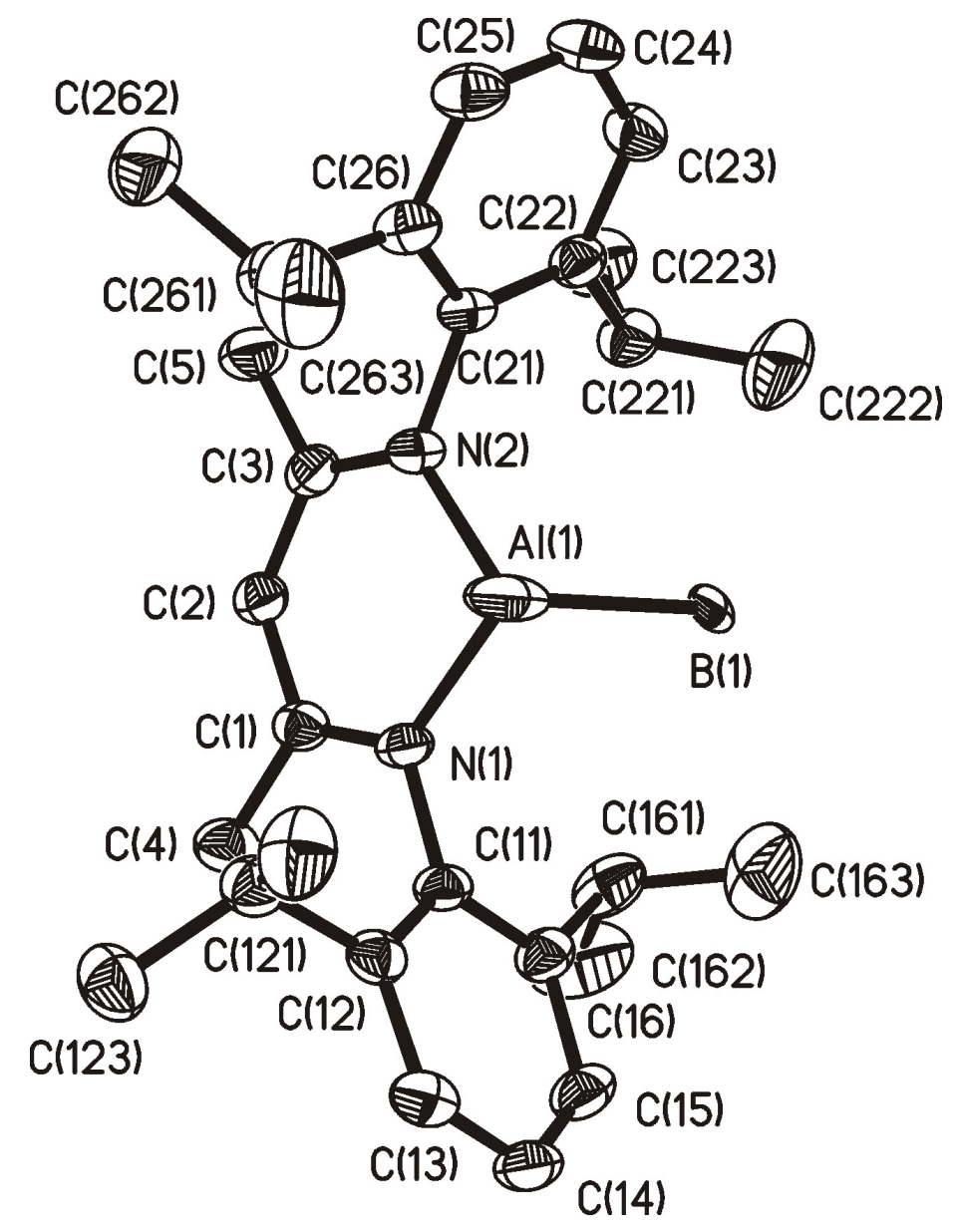

Abb. 6. Struktur von 20 im Kristall 
Tab.9. Ausgewählte Bindungslängen $[\AA]$ und -winkel $\left[{ }^{\circ}\right]$ von 20

\begin{tabular}{lllr}
\hline $\mathrm{Al}(1)-\mathrm{B}(1)$ & $1.953(4)$ & $\mathrm{N}(1)-\mathrm{Al}(1)-\mathrm{B}(1)$ & $115.11(16)$ \\
$\mathrm{Al}(1)-\mathrm{N}(1)$ & $1.896(2)$ & $\mathrm{N}(2)-\mathrm{Al}(1)-\mathrm{B}(1)$ & $115.73(15)$ \\
$\mathrm{Al}(1)-\mathrm{N}(2)$ & $1.901(2)$ & $\mathrm{N}(1)-\mathrm{Al}(1)-\mathrm{N}(2)$ & $96.86(10)$ \\
$\mathrm{C}(1)-\mathrm{C}(2)$ & $1.398(3)$ & & \\
$\mathrm{C}(2)-\mathrm{C}(3)$ & $1.398(4)$ & & \\
$\mathrm{N}(1)-\mathrm{C}(1)$ & $1.330(3)$ & & \\
$\mathrm{N}(2)-\mathrm{C}(3)$ & $1.335(3)$ & & \\
\hline
\end{tabular}

Verbindung 20 kristallisiert monoklin in der Raumgruppe $\mathrm{P} 2{ }_{1} / \mathrm{n}$ mit vier unabhängigen Formeleinheiten pro Elementarzelle. Zentrale Einheit der Struktur ist ein planarer sechsatomiger $\mathrm{AlN}_{2} \mathrm{C}_{3}$-Ring. Das Aluminiumatom ist mit zwei Stickstoffatomen des (Dipp) ${ }_{2}$ NacNac-Liganden und mit einem Wasserstoffatom verbunden. Zusätzlich ist eine $\mathrm{BH}_{2}$-Gruppe terminal am Aluminium gebunden. Die Koordinationssphäre des Aluminiums ist verzerrt tetraedrisch. Die Bindungslängen im $\mathrm{AlN}_{2} \mathrm{C}_{3}$-Ring sind vergleichbar mit denen, die für (Dipp) ${ }_{2} \mathrm{NacNacAlH}_{2}$ ermittelt wurden. ${ }^{[135]}$ Der Bor-Aluminium-Bindungsabstand ist nicht vergleichbar, da bisher keine Literaturstellen über formale Bor(II)-Aluminium(II)-Bindungen literaturbekannt sind. Cowley et al. berichteten über eine Aluminium(I)-Bor(III)-Verbindung, ${ }^{[136]}$ in der der Aluminium-Bor-Bindungsabstand 2.169(3) A beträgt. Im 3-Et-3-alumina-1,2dicarba-closo-dodecaboran beträgt der Aluminium-Bor-Abstand im Mittel 2.173(7) $\AA^{[137]}$ Beide gefundenen Werte sind länger, als sie in $\mathbf{2 0}$ gefunden werden.

\subsubsection{Versuch zur Darstellung von (Dipp) ${ }_{2} \mathrm{NacNacSnF}$}

Wie erwähnt, sind Hauptgruppenfluoride einfach mit $\mathrm{Me}_{3} \mathrm{SnF}$ zu erhalten. ${ }^{[22,23]}$ In diesem Abschnitt sollte versucht werden, analog dem synthetisierten (Dipp) ${ }_{2} \mathrm{NacNacGeF}^{[47]}$ das (Dipp) ${ }_{2} \mathrm{NacNacSnF}$ darzustellen. Es wurden dazu zwei Wege beschritten. (Schema 20.) 


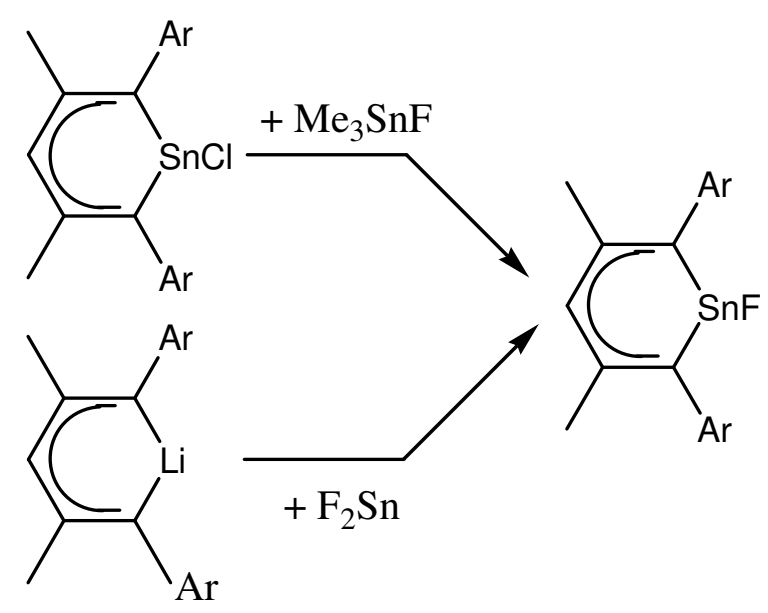

Schema 20.

Bei den Umsetzungen wurden in beiden Fällen nach Aufarbeitung der Reaktionslösungen die Ausgangssubstanzen wiedergewonnen. Auch unter drastischeren Bedingungen ließ sich keine Reaktion dieser Substanzen beobachten.

\subsubsection{Darstellung von $(t-B u S n)_{4} S_{6}(21)$}

Durch Umsetzung von (Dipp) ${ }_{2} \mathrm{NacNacGeCl}$ waren nicht nur das Hydrid sondern auch Oxidationsreaktionen mit elementaren Schwefel, Selen und Tellur zu beobachten. Es wurden Germanium(IV)-Verbindungen erhalten, in welchen Doppelbindungen zu Elementen der 16. Gruppe als auch Bindungen zu Halogenatomen erhältlich sind. ${ }^{[60]}$ Abschließend sollte nun untersucht werden, ob solche Verbindungen auch mit Zinn darstellbar sind.

Eine Suspension aus (Dipp) ${ }_{2} \mathrm{NacNacSn}(t-\mathrm{Bu})$ und Schwefel in Toluol wurde für drei Stunden unter Rückfluss gerührt. Nach Aufarbeitung wurde das Rohprodukt mit $n$ Hexan aufgenommen. Nach drei Tagen bei $-26{ }^{\circ} \mathrm{C}$ kristallisierte 17 aus, welches einen Schmelzpunkt von $210{ }^{\circ} \mathrm{C}$ hat. 


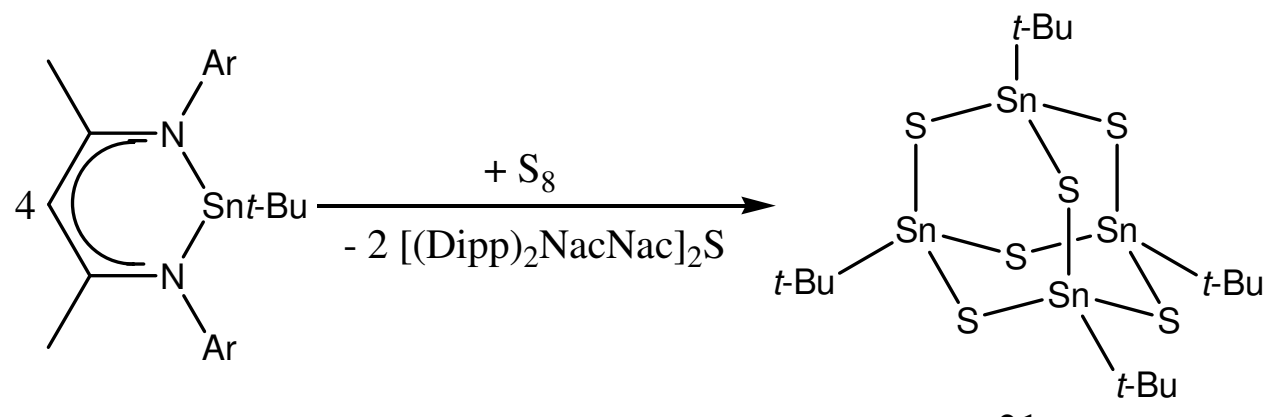

21

Schema 21.

Das Massenspektrum zeigt, dass unter (Dipp) ${ }_{2} \mathrm{NacNac-Abspaltung} \mathrm{eine}$ adamantananaloge Struktur, ähnlich wie sie z. B. Haas et al. bei der Umsetzung von $\mathrm{RSnCl}_{3}$ mit $\left(\mathrm{Me}_{3} \mathrm{Si}\right)_{2} \mathrm{~S}$ beobachten konnten, ${ }^{[138]}$ entstanden ist. Der Peak bei $\mathrm{m} / \mathrm{z} 838$ lässt sich dem Molekülion abzüglich einer $t$-Bu-Gruppe zuordnen. Im ${ }^{1} \mathrm{H}-\mathrm{NMR}$ Spektrum ist lediglich ein Singulett bei $\delta 1.29 \mathrm{ppm}$ als Resonanz für die $t$-Bu-Protonen zu finden. 


\section{Zusammenfassung}

Im ersten Teil dieser Arbeit sollten die terminal gebundenen Methyl-Gruppen des Iminoalans $\left[\mathrm{MeAlN}\left(2,6-i-\mathrm{Pr}_{2} \mathrm{C}_{6} \mathrm{H}_{3}\right)\right]_{3}$ (1) durch Halogenatome ausgetauscht werden. Durch Reaktion von 1 mit $\mathrm{Me}_{3} \mathrm{SnF}$ konnte ein Gemisch aus [FAlN(2,6-i- $\left.\left.\mathrm{Pr}_{2} \mathrm{C}_{6} \mathrm{H}_{3}\right)\right]_{3} \cdot 3$ THF (7) und $\left[\mathrm{Me}_{2} \mathrm{SnN}\left(2,6-i-\mathrm{Pr}_{2} \mathrm{C}_{6} \mathrm{H}_{3}\right)\right]_{2}(\mathbf{8})$ isoliert werden.

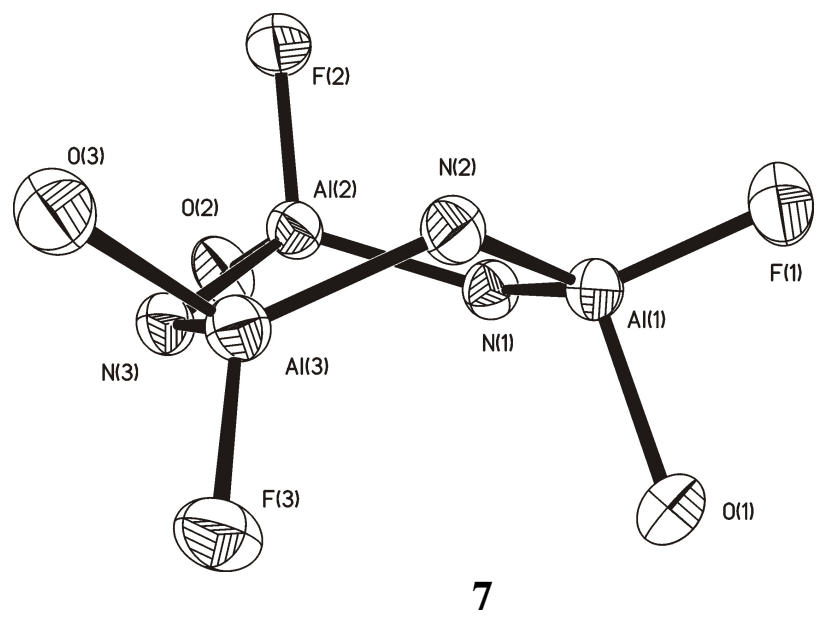

Kristalle für eine Röntgenstrukturanalyse von 7 waren aus THF/n-Hexan (1:5) und Kristalle von $\mathbf{8}$ aus THF/Toluol (1:10) erhältlich, offensichtlich ist $\mathbf{8}$ ein Nebenprodukt aus der Reaktion von 7 mit $\mathrm{Me}_{3} \mathrm{SnF}$.

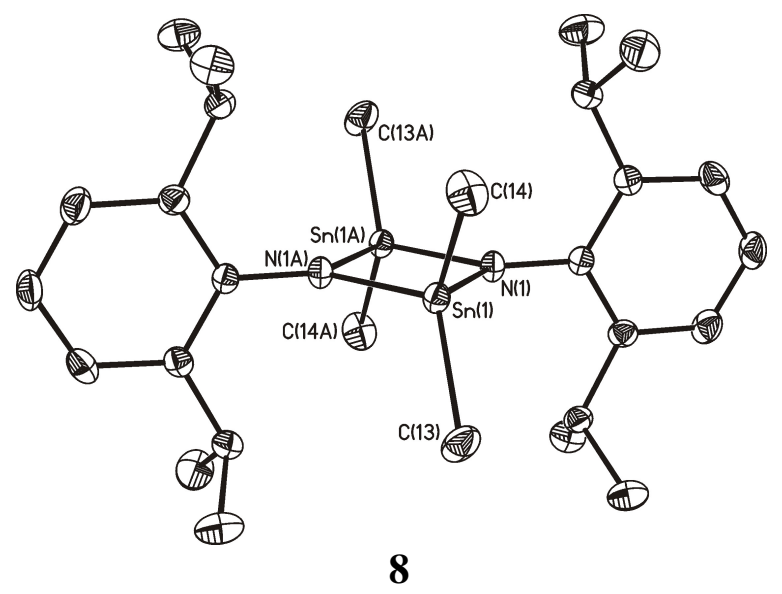


Wurde $n-\mathrm{Bu}_{4} \mathrm{~N}\left(\mathrm{HF}_{2}\right)$ als Fluorierungsreagenz verwendet, so konnte das literaturbekannte ${ }^{[100]} n-\mathrm{Bu}_{4} \mathrm{~N}\left[\mathrm{Me}_{2} \mathrm{AlF}_{2}\right]$ erhalten werden. Durch Verwendung von Borhalogenid-Lösungen konnte $\mathbf{1}$ in die jeweiligen Halogenderivate überführt werden.

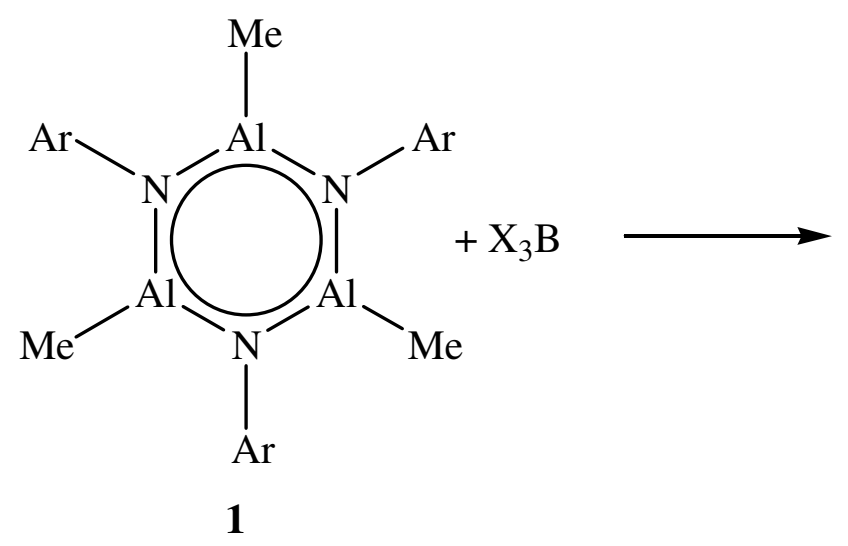

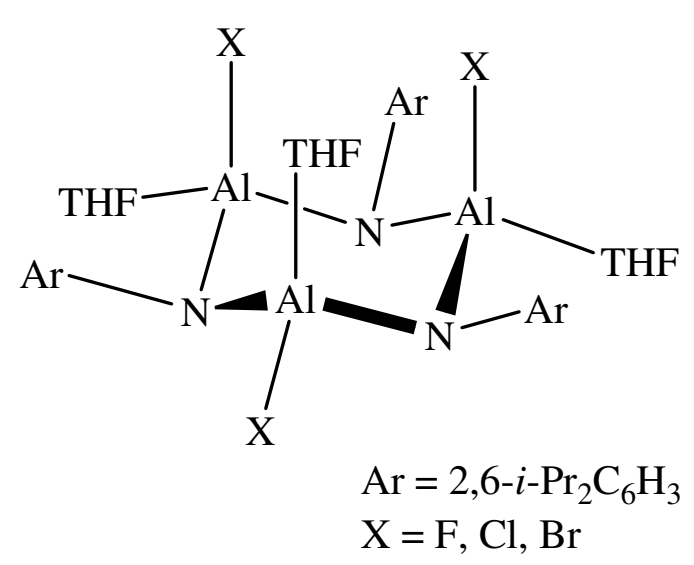

Die Umsetzung von 1 mit $\mathrm{F}_{3} \mathrm{~B} \cdot \mathrm{OEt}_{2}$ als Fluorierungsreagenz ergab Verbindung 7. Durch Umsetzungen mit $\mathrm{Cl}_{3} \mathrm{~B}-$ bzw. $\mathrm{Br}_{3} \mathrm{~B}$-Lösungen waren $[\mathrm{ClAlN}(2,6-i$ $\left.\left.\mathrm{Pr}_{2} \mathrm{C}_{6} \mathrm{H}_{3}\right)\right]_{3} \cdot 3$ THF (9) und $\left[\mathrm{BrAlN}\left(2,6-i-\mathrm{Pr}_{2} \mathrm{C}_{6} \mathrm{H}_{3}\right)\right]_{3} \cdot 3$ THF (10) darstellbar. Versuche zur Darstellung des analogen Iod-substituierten Alumazens blieben ebenso erfolglos wie die Darstellung des Wasserstoff-substituierten Derivates. Bei letzterer Reaktion konnte lediglich $\left[\mathrm{MeAlN}\left(2,6-i-\mathrm{Pr}_{2} \mathrm{C}_{6} \mathrm{H}_{3}\right)\right]_{3} \cdot 3 \mathrm{THF}(\mathbf{1 1})$ isoliert und identifiziert werden.

Der zweite Abschnitt dieser Arbeit hatte die Darstellung von monoorganylsubstituierten Germaniumtrihalogeniden zur Aufgabe. Als Ligandensystem wurde der Terphenyl-Ligand 2,6-Bis(2`,6`-dimethylphenyl)phenyl ausgewählt. Dieser konnte durch einfache Umsetzung von $n$-BuLi und 2,6-Bis(2‘,6`dimethylphenyl)-1-iodbenzol (12) und anschließender Umsetzung mit einem Germaniumtetrahalogenid eingeführt werden. 12 ließ sich im Rahmen dieser Arbeit strukturell charakterisieren. 


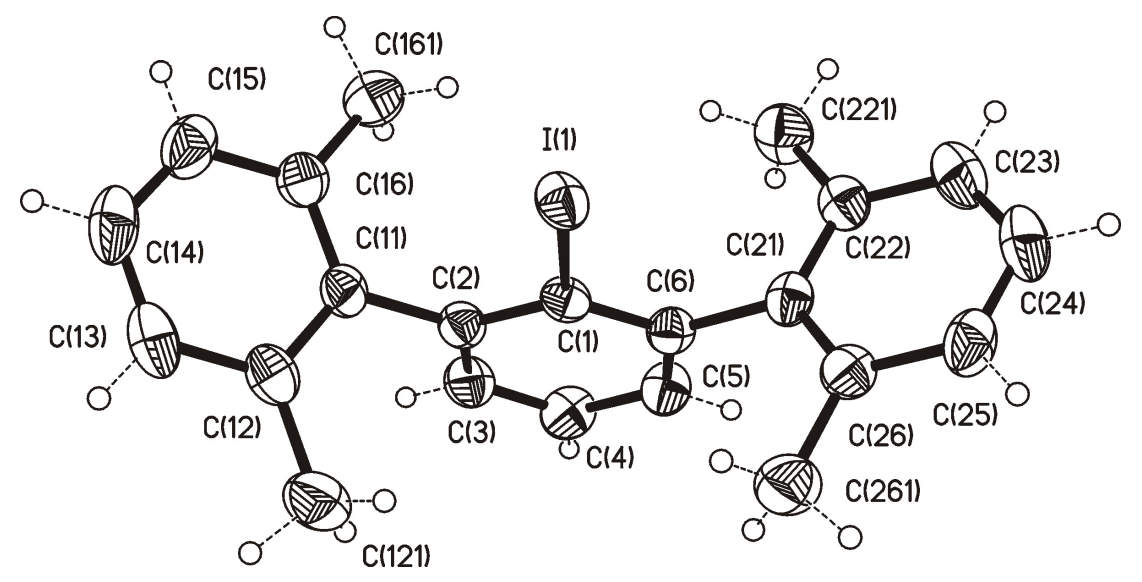

Durch stöchiometrische Reaktionen mit dem Lithiumsalz von 12 waren 2,6$\operatorname{Bis}\left(2^{`}, 6^{6}\right.$-dimethylphenyl)-phenylgermaniumtrichlorid (13), $2,6-\operatorname{Bis}\left(2^{`}, 6^{‘}-\right.$ dimethylphenyl)-phenylgermaniumtribromid (14) und 2,6-Bis(2‘,6‘-dimethylphenyl)phenylgermaniumtrichlorid (15) in hohen Ausbeuten darstellbar. Verbindungen 13 und 14 konnten strukturell charakterisiert werden.

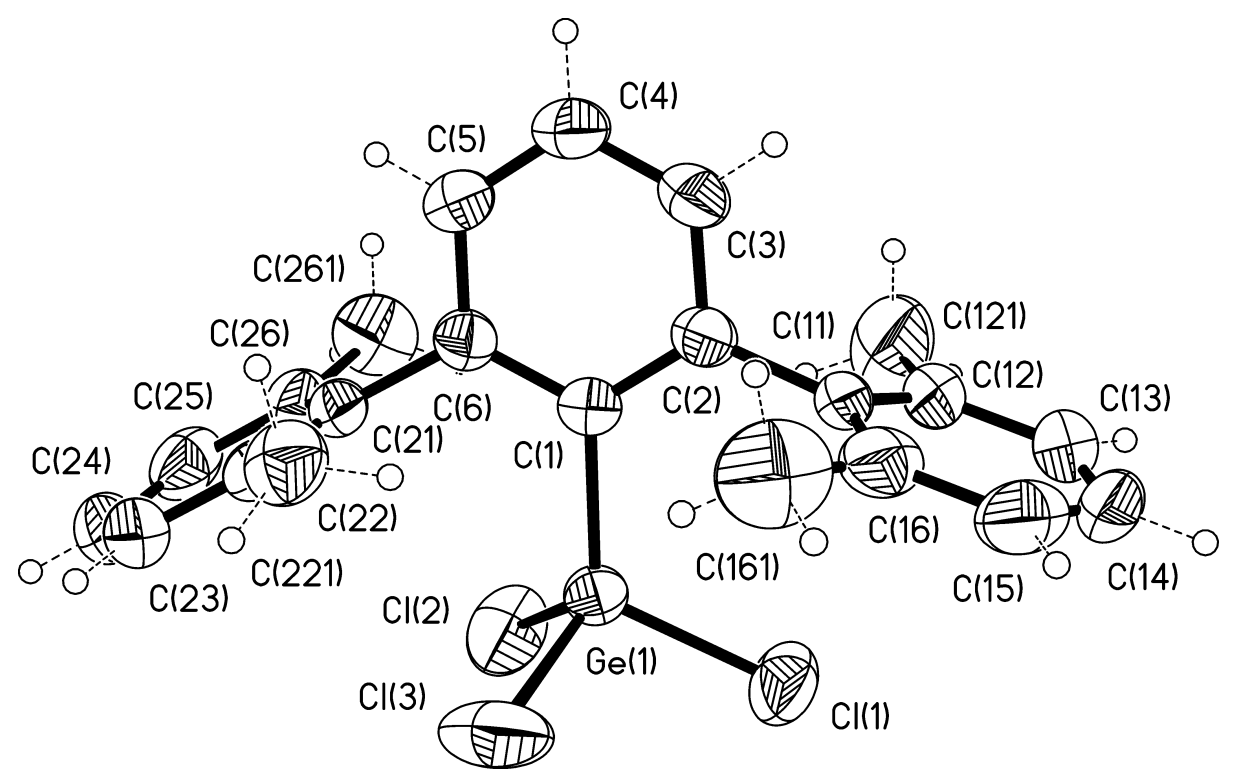




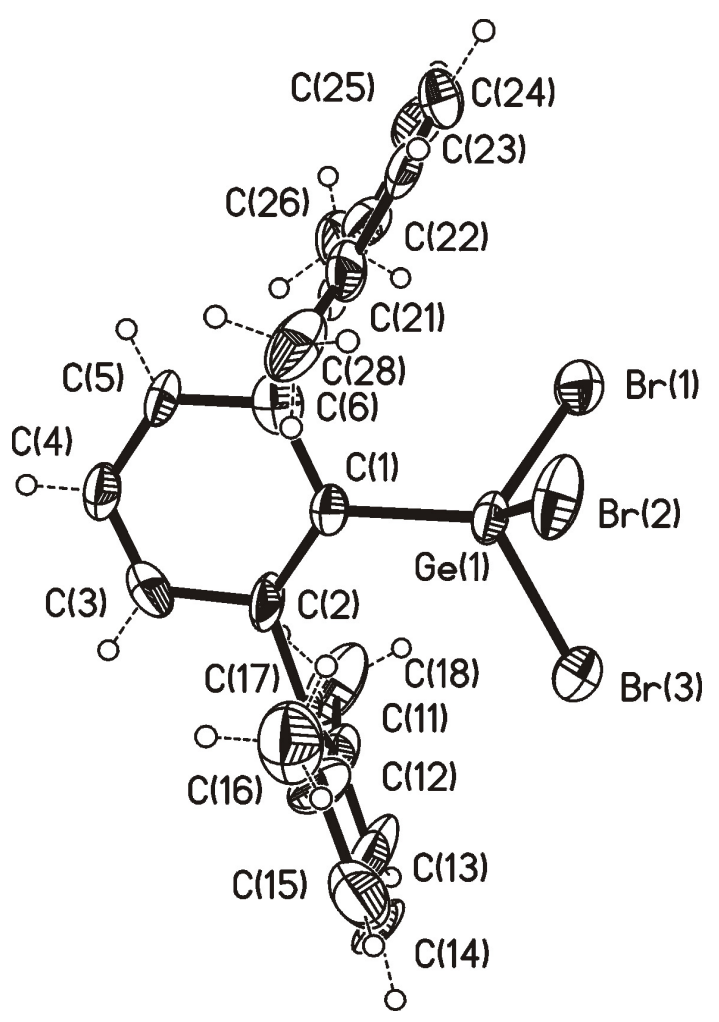

Mit $\mathrm{Me}_{3} \mathrm{SnF}$ als Fluorierungsmittel konnte 13 fluoriert und das 2,6-Bis(2‘,6`dimethylphenyl)phenylgermaniumtrifluorid (16) erhalten werden. Auf gleiche Weise wurde DippN($\left(\mathrm{SiMe}_{3}\right) \mathrm{GeF}_{3}$ (17) dargestellt. Verbindungen 16 und 17 sind flüssige Substanzen mit relativ hohen Siedepunkten. Sie konnten sowohl Massenspektrometrisch, NMR-spektroskopisch als auch durch Elementaranalyse charakterisiert werden.

Im dritten Teil der Arbeit sollten kondensationsstabile Ge-OH-Verbindungen dargestellt werden. Dazu wurde $\operatorname{DippN}\left(\mathrm{SiMe}_{3}\right) \mathrm{GeBr}_{3}$ bzw. Verbindung 13 verwendet, um Kondensationsreaktionen $\mathrm{zu}$ verhindern. Jedoch zeigten alle Versuche, dass als Produkte nur adamantanartige Gerüste (Verbindungen 18 und 19) als Hauptprodukte isoliert werden konnten. Die IR-Spektren indizieren allerdings, dass Verbindungen mit OH-Gruppen entstanden sind. Versuche, diese Verbindungen zu trennen, schlugen fehl. 
<smiles>[R]C12OC([R])(O1)OC([R])(OC1([R])OC1([R])O)O2</smiles>

18: $\mathrm{R}=2,6-i-\mathrm{Pr}_{2} \mathrm{C}_{6} \mathrm{H}_{3} \mathrm{~N}\left(\mathrm{SiMe}_{3}\right)$;

19: $\mathrm{R}=\left(2,6-\mathrm{Me}_{2} \mathrm{C}_{6} \mathrm{H}_{3}\right)_{2} \mathrm{C}_{6} \mathrm{H}_{3}$

Abschließend sollten in dieser Arbeit Verbindungen mit niedervalenten Elementen der 14. Gruppe auf ihre Reaktivität untersucht werden. Durch Reaktion von (Dipp) ${ }_{2} \mathrm{NacNacGe}\left(\mathrm{BH}_{3}\right) \mathrm{H}$ mit $\mathrm{H}_{3} \mathrm{Al} \cdot \mathrm{NMe}_{3}$ wurde $\quad$ (Dipp) ${ }_{2} \mathrm{NacNacAlHBH}_{2} \quad$ (20) isolierbar, wobei erstmalig eine Aluminium-Bor-Bindung mit der formalen Oxidationsstufe II für beide Elemente bestimmt werden konnte.

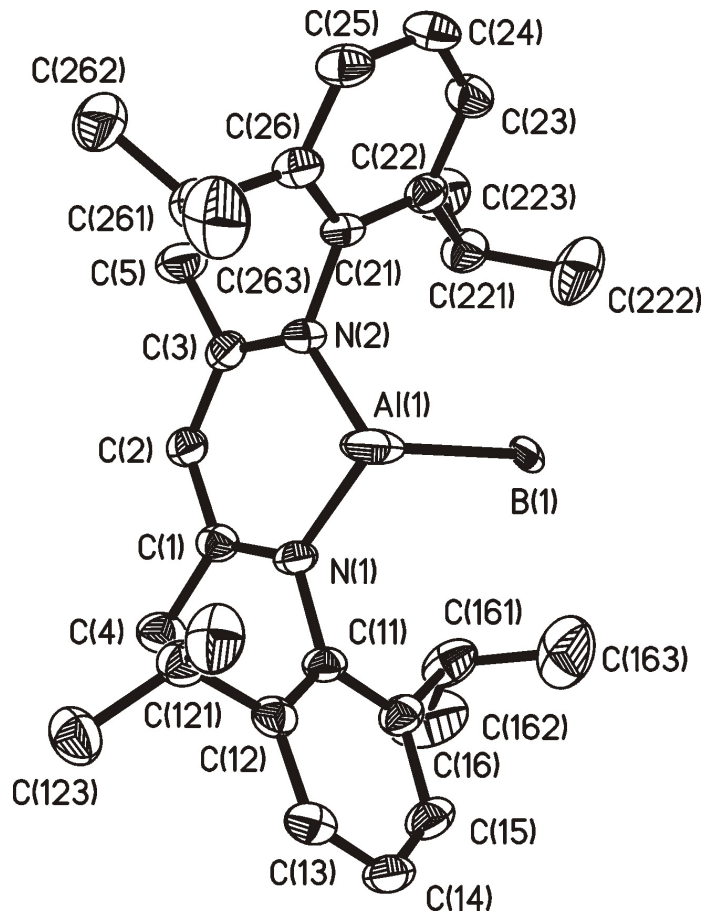

20

Außerdem konnte in dieser Arbeit gezeigt werden, dass durch Umsetzung von (Dipp) ${ }_{2} \mathrm{NacNacSn}(t-\mathrm{Bu})$ mit elementarem Schwefel nicht eine Addition eines 
Schwefelatoms erfolgt, wie sie für die entsprechende Germanium-Verbindungen gefunden wird, sondern die Abspaltung des (Dipp) ${ }_{2} \mathrm{NacNac-Liganden} \mathrm{und} \mathrm{die} \mathrm{Bildung}$ eines adamantanalogen Zinn-Schwefel-Gerüstes fand statt. 


\section{Experimenteller Teil}

\subsubsection{Allgemeine Arbeitstechniken}

Sämtliche Versuche wurden unter Luft- und Feuchtigkeitsausschluss in ausgeheizten und im Vakuum abgekühlten Glasgeräten unter einer getrockneten $\left(\mathrm{P}_{4} \mathrm{O}_{10}\right)$ und sauerstofffreien Sickstoffatmosphäre durchgeführt. Die verwendeten Lösungsmittel wurden nach Literaturmethoden ${ }^{[139]}$ getrocknet, vor Gebrauch unter $\mathrm{N}_{2}$-Atmosphäre destilliert und durch mehrmaliges Anlegen von Vakuum und Spülen mit Stickstoff entgast.

\subsubsection{Physikalische Messmethoden}

Schmelz- und Zersetzungspunkte der Substanzen wurden in unter Stickstoff zugeschmolzenen Glaskapillaren mit den Geräten HWS-SG 3000, Bühler SPA-1 und Büchi B-540 gemessen.

Elementaranalysen wurden im Analytischen Labor des Instituts für Anorganische Chemie der Georg-August-Universität Göttingen durchgeführt. Elementaranalysen von Metallstickstoffverbindungen der 13. Gruppe sind jedoch oft relativ ungenau bzw. nicht aussagekräftig. ${ }^{[129]}$ Ursache hierfür ist die Bildung von Metallnitriden oder -carbiden.

NMR-Spektren wurden auf den Geräten AM 200, Avance 200, AM 250, AM 300, AS 400 und Avance 500 Spektrometern der Fa. Bruker aufgenommen. Soweit nicht anders angegeben, wurde bei Raumtemperatur gemessen. Lösungsmittel und Betriebsfrequenzen sind bei den Versuchsbeschreibungen aufgeführt. Die chemischen Verschiebungen sind als $\delta$ Werte in ppm angegeben, wobei negative Vorzeichen Hochfeldverschiebung und positive Vorzeichen Tieffeldverschiebung in Bezug auf den jeweiligen Standard bedeuten. Die Multiplizitäten werden wie folgt angegeben: $\mathrm{s}=$ Singulett, $\mathrm{d}=$ Dublett, $\mathrm{t}=$ Triplett, $\mathrm{q}=$ Quartett, sept $=$ Septett, $\mathrm{m}=$ Multiplett, br $=$ breit. Die Kopplungskonstanten $J$ sind in $\mathrm{Hz}=$ Hertz angegeben. Als externe Standards wurden TMS $\left({ }^{1} \mathrm{H}-,{ }^{13} \mathrm{C}-\right.$ und $\left.{ }^{29} \mathrm{Si}_{-\mathrm{NMR}}\right), \mathrm{F}_{3} \mathrm{~B} \cdot \mathrm{OEt}_{2}$ $\left({ }^{11} \mathrm{~B}-\mathrm{NMR}\right), \mathrm{CFCl}_{3}\left({ }^{19} \mathrm{~F}-\mathrm{NMR}\right)$ und $\mathrm{Me}_{4} \mathrm{Sn}\left({ }^{119} \mathrm{Sn}-\mathrm{NMR}\right)$. 
Massenspektren wurden mit den Geräten Finnigan MAT 8200, Varian MAT CH 5 und Finnigan MAT 95 aufgenommen. Die verwendete Ionisierungsmethode war Elektronenstoßionisation (EI). Bei Molekülfragmenten mit charakteristischem Isotopenmuster ist nur das stärkste Signal mit seiner relativen Intensität angegeben.

IR-Spektren der Verbindungen wurden als Nujolverreibungen zwischen KBr-Platten auf einem BIO-RAD Digilab FTS-7-Spektrometer aufgenommen. Die Bandenintensitäten werden durch folgende Abkürzungen wiedergegeben: $\mathrm{sst}=\mathrm{sehr}$ stark, $\mathrm{st}=$ stark, $\mathrm{m}=$ mittel, $\mathrm{s}$ = schwach. Sofern es sich nicht um charakteristische Schwingungen handelt, werden nur mittelstarke, starke und sehr starke Banden angegeben. Die Wellenzahlen $\tilde{v}$ sind in $\mathrm{cm}^{-1}$ angegeben.

Einkristall-Röntgenstrukturanalysen wurden auf einem Stoe-Siemens-HuberVierkreisdiffraktometer, Stoe IPDS II und STOE AED 2 Vierkreisdiffraktometern mit monochromatischer Mo-K $\mathrm{K}_{\alpha}$-Strahlung $(\lambda=0.71073 \AA)$ in Öltropfen eingebetteten und schockgekühlten Kristallen durchgeführt. Die Stukturverfeinerung erfolgte in allen Fällen nach der Methode Vollmatrix Least-Squares an $F^{2}$ (SHELXS-97 ${ }^{[140]}$, SHELXL-97 ${ }^{[141]}$ ). Alle Nichtwasserstoffatome wurden anisotrop verfeinert und die Wasserstoffatome dem Modell auf geometrisch berechneten Positionen hinzugefügt und verfeinert.

\section{Darstellung der Ausgangsverbindungen:}
$\left[\mathrm{MeAlN}\left(2,6-i-\mathrm{Pr}_{2} \mathrm{C}_{6} \mathrm{H}_{3}\right)\right]_{3} ;{ }^{[87]}$
$\mathrm{Me}_{3} \mathrm{SnF} ;{ }^{[142]}$
$\mathrm{PhSi}(\mathrm{C} \equiv \mathrm{CH})_{3},{ }^{[114]}$
Dichloriodbenzol; ${ }^{[143]}$
$\operatorname{DippN}\left(\mathrm{SiMe}_{3}\right) \mathrm{GeBr}_{3} ;{ }^{[127]}$
$\left[\mathrm{DippN}\left(\mathrm{SiMe}_{3}\right) \mathrm{Ge}\left(\mathrm{NH}_{2}\right)_{2}\right]_{2} \mathrm{NH} ;{ }^{[127]}$
$(\text { Dipp })_{2} \mathrm{NacNacLi}{ }^{[144]}$
$(\text { Dipp })_{2} \mathrm{NacNacGeH} \cdot \mathrm{BH}_{3} ;{ }^{[47]}(\mathrm{Dipp})_{2} \mathrm{NacNacSnCl} ;{ }^{[47]}(\mathrm{Dipp})_{2} \mathrm{NacNacSn}(t-\mathrm{Bu}) .{ }^{[47]}$ 


\subsection{Umsetzungen von $\left[\mathrm{MeAIN}\left(2,6-i-\operatorname{Pr}_{2} \mathrm{C}_{6} \mathrm{H}_{3}\right)\right]_{3}(\mathbf{1})$}

5.1.1. Darstellung von $\left[\mathrm{FAlN}\left(2,6-i-\mathrm{Pr}_{2} \mathrm{C}_{6} \mathrm{H}_{3}\right)\right]_{3} \cdot 3 \mathrm{THF}(7)$ und $\left[\mathrm{Me}_{2} \mathrm{SnN}\left(2,6-i-\mathrm{Pr}_{2} \mathrm{C}_{6} \mathrm{H}_{3}\right)\right]_{2}(\mathbf{8})$ :

$\mathrm{Zu}$ einer Suspension von $1.11 \mathrm{~g}(6.0 \mathrm{mmol}) \mathrm{Me}_{3} \mathrm{SnF}$ in $40 \mathrm{ml} \mathrm{THF}$ wurde bei Raumtemperatur eine Lösung aus $1.30 \mathrm{~g}$ (2.0 mmol) 1 in $40 \mathrm{ml}$ THF gegeben. Anschließend wurde das Gemisch für weitere 12 Stunden bei Raumtemperatur gerührt, wobei die ungelösten Bestandteile komplett in Lösung gingen. Danach wurden alle flüchtigen Bestandteile im Vakuum entfernt.

a) Nach Zugabe von $30 \mathrm{ml} \mathrm{THF} / n$-Hexan (1:5) und Kristallisation bei $-26{ }^{\circ} \mathrm{C}$ konnten $1.23 \mathrm{~g}$ (1.49 mmol, $70 \%) 7$ als farbloser kristalliner Feststoff erhalten werden.

Schmelzpunkt: $>300^{\circ} \mathrm{C}$

${ }^{1} \mathrm{H}-\mathrm{NMR}\left(250.13 \mathrm{MHz}, \mathrm{C}_{6} \mathrm{D}_{6}\right)$ :

$\delta 0.77\left(\mathrm{~m}, 4 \mathrm{H}, \mathrm{OCH}_{2} \mathrm{CH}_{2}\right), 1.02\left(\mathrm{~m}, 8 \mathrm{H}, \mathrm{OCH}_{2} \mathrm{CH}_{2}\right), 1.25\left(\mathrm{~d}, J_{\mathrm{H}, \mathrm{H}}=7 \mathrm{~Hz}, 12 \mathrm{H}, \mathrm{CH}\left(\mathrm{CH}_{3}\right)_{2}\right)$, $1.41\left(\mathrm{~d}, J_{\mathrm{H}, \mathrm{H}}=7 \mathrm{~Hz}, 6 \mathrm{H}, \mathrm{CH}\left(\mathrm{CH}_{3}\right)_{2}\right), 1.50\left(\mathrm{~d}, J_{\mathrm{H}, \mathrm{H}}=7 \mathrm{~Hz}, 6 \mathrm{H}, \mathrm{CH}\left(\mathrm{CH}_{3}\right)_{2}\right), 1.65\left(\mathrm{~d}, J_{\mathrm{H}, \mathrm{H}}=7\right.$ $\left.\mathrm{Hz}, 6 \mathrm{H}, \mathrm{CH}\left(\mathrm{CH}_{3}\right)_{2}\right), 1.73\left(\mathrm{~d}, \mathrm{~J}_{\mathrm{H}, \mathrm{H}}=7 \mathrm{~Hz}, 6 \mathrm{H}, \mathrm{CH}\left(\mathrm{CH}_{3}\right)_{2}\right), 3.20\left(\mathrm{~m}, 4 \mathrm{H}, \mathrm{OCH}_{2} \mathrm{CH}_{2}\right), 3.55$ (m, $\left.4 \mathrm{H}, \mathrm{OCH}_{2} \mathrm{CH}_{2}\right), 3.83\left(\mathrm{~m}, 4 \mathrm{H}, \mathrm{OCH}_{2} \mathrm{CH}_{2}\right), 4.40\left(\mathrm{~m}, 4 \mathrm{H}, \mathrm{CH}\left(\mathrm{CH}_{3}\right)_{2}\right), 4.75$ (sept, $J_{\mathrm{H}, \mathrm{H}}=7$ $\left.\mathrm{Hz}, 2 \mathrm{H}, \mathrm{CH}\left(\mathrm{CH}_{3}\right)_{2}\right), 7.00(\mathrm{~m}, 9 \mathrm{H}$, arom. $H)$ ppm.

${ }^{19}$ F-NMR (188.28, MHz, $\left.\mathrm{C}_{6} \mathrm{D}_{6}\right)$ :

$\delta-166.3(2 \mathrm{~F}),-153.9(1 \mathrm{~F}) \mathrm{ppm}$.

IR (KBr, Nujol): $\tilde{v}=3045$ (m), 1585 (m), 1422 (sst), 1358 (st), 1310 (st), 1260 (m), 1231 (sst), 1183 (sst), 1107 (st), 1039 (m), 1010 (m), 958 (m), 918 (m), 891 (st),861 (st), 834 (sst), 789 (sst), 746 (m), 723 (m), 633 (m), 602 (m), 546 (s), 457 (st) cm ${ }^{-1}$.

MS (EI, $m / z,(\%)): 558$ [M-2Dipp] (20), 162 [DippH] (100). 
Elementaranalyse für $\mathrm{C}_{48} \mathrm{H}_{75} \mathrm{Al}_{3} \mathrm{~F}_{3} \mathrm{~N}_{3} \mathrm{O}_{3}(880.05 \mathrm{~g} / \mathrm{mol})$ :

$\begin{array}{llll} & \mathrm{C} & \mathrm{H} & \mathrm{F} \\ \text { Ber.: } & 65.51 & 8.59 & 6.48, \\ \text { Gef.: } & 65.6 & 8.4 & 6.5 \% .\end{array}$

b) Wurde das Gemisch hingegen in $30 \mathrm{ml}$ einer Lösung aus THF/Toluol (1:10) gelöst, kristallisierte bei $0{ }^{\circ} \mathrm{C} 0.23 \mathrm{~g}(0.35 \mathrm{mmol}, 2.3 \%) 8$ als farbloser kristalliner Feststoff.

Schmelzpunkt: $280^{\circ} \mathrm{C}$.

${ }^{1} \mathrm{H}-\mathrm{NMR}\left(200.13 \mathrm{MHz}, \mathrm{C}_{6} \mathrm{D}_{6}\right)$ :

$\delta 0.40\left(\mathrm{~s}, J_{\mathrm{H}, \mathrm{Sn}}=56 \mathrm{~Hz}\left({ }^{117} \mathrm{Sn}\right) / 58 \mathrm{~Hz}\left({ }^{119} \mathrm{Sn}\right), 12 \mathrm{H}, \mathrm{Sn}\left(\mathrm{CH}_{3}\right)_{2}\right), 1.28\left(\mathrm{~d}, J_{\mathrm{H}, \mathrm{H}}=6.9 \mathrm{~Hz}, 24\right.$ $\left.\mathrm{H}, \mathrm{CH}\left(\mathrm{CH}_{3}\right)_{2}\right), 4.27$ (sept, $\left.J_{\mathrm{H}, \mathrm{H}}=6.9 \mathrm{~Hz}, 4 \mathrm{H}, \mathrm{CH}\left(\mathrm{CH}_{3}\right)_{2}\right), 7.12(\mathrm{~m}, 6 \mathrm{H}$, arom. H) ppm.

${ }^{13} \mathrm{C}-\mathrm{NMR}\left(125.76 \mathrm{MHz}, \mathrm{C}_{6} \mathrm{D}_{6}\right)$ :

$\delta 2.5\left(J_{\mathrm{C}, \mathrm{Sn}}=360 \mathrm{~Hz}\left({ }^{117} \mathrm{Sn}\right) / 376 \mathrm{~Hz}\left({ }^{119} \mathrm{Sn}\right), \mathrm{SnCH} \mathrm{H}_{3}\right), 25.4\left(\mathrm{CH}\left(\mathrm{CH}_{3}\right)_{2}\right), 27.3\left(\mathrm{CH}\left(\mathrm{CH}_{3}\right)_{2}\right)$, 122.3 (arom. C), 123.4 (arom. C), 147.5 (arom. C), 148.0 (arom. C) ppm.

${ }^{119} \mathrm{Sn}-\mathrm{NMR}\left(186.50 \mathrm{MHz}, \mathrm{C}_{6} \mathrm{D}_{6}\right)$ :

$\delta 114.67\left(\mathrm{~s}, J_{\mathrm{Sn}, \mathrm{Sn}}=69 \mathrm{~Hz}\right) \mathrm{ppm}$.

MS (EI, m/z, (\%)): 648 [M] (40), 633 [M-Me] (80), 324 [M/2] (90) 161 [Dipp] (100).

Elementaranalyse für $\mathrm{C}_{28} \mathrm{H}_{46} \mathrm{~N}_{2} \mathrm{Sn}_{2}(648.08 \mathrm{~g} / \mathrm{mol})$ :

$\begin{array}{llll} & \mathrm{C} & \mathrm{H} & \mathrm{N} \\ \text { Ber.: } & 36.63 & 7.15 & 4.32, \\ \text { Gef.: } & 35.9 & 7.4 & 4.5 \% .\end{array}$




\subsubsection{Allgemeine Vorschrift zur Umsetzung von 1 mit $n-\mathrm{Bu}_{4} \mathrm{~N}\left(\mathrm{HF}_{2}\right)$ :}

Der Wert in den Klammern bezieht sich immer auf das Stöchiometrieverhältnis, welches bei der Reaktion zur Anwendung kam. So ist mit a das Verhältnis 1 zu $n-\mathrm{Bu}_{4} \mathrm{~N}\left(\mathrm{HF}_{2}\right)$ 1:1, mit b das Verhältnis 1:2 und mit $\mathbf{c}$ das Verhältnis 1:3 gemeint.

Zu $0.67 \mathrm{~g}(1.03 \mathrm{mmol}) 1$ a) (b: $0.67 \mathrm{~g}, 1.03 \mathrm{mmol}$; c: $0.69 \mathrm{~g}, 1.06 \mathrm{mmol})$ in $40 \mathrm{ml}$ THF wurde bei Raumtemperatur $0.65 \mathrm{ml}$ a) (b: $1.34 \mathrm{ml}$; c: $2.06 \mathrm{ml}$ ) einer $1.54 \mathrm{M}$ Lösung aus $n$ $\mathrm{Bu}_{4} \mathrm{~N}\left(\mathrm{HF}_{2}\right)$ in THF tropfenweise gegeben. 12 Stunden Reaktionszeit, Entfernen des Lösungsmittels im Vakuum kristallisierten bei $-26{ }^{\circ} \mathrm{C}$ nach $\mathbf{a}$ : acht Wochen, b: fünf Wochen, c: drei Wochen rautenförmige Kristalle von $n-\mathrm{Bu}_{4} \mathrm{~N}\left(\mathrm{Me}_{2} \mathrm{AlF}_{2}\right)$ aus $(\mathbf{a}: 0.23 \mathrm{~g} ; \mathbf{b}: 0.34 \mathrm{~g}$; : $0.38 \mathrm{~g})$.

Die spektroskopischen Daten entsprechen den in der Literatur ${ }^{[100]}$ angegebenen.

\subsubsection{Umsetzung von 1 mit $_{3} \mathrm{~B} \cdot \mathrm{OEt}_{2}$ :}

$\mathrm{Zu} 0.79 \mathrm{~g}(1.20 \mathrm{mmol}) \mathbf{1}$ in $20 \mathrm{ml}$ THF wurden bei Raumtemperatur $1.20 \mathrm{ml}(1.20$ mmol) einer $1.0 \mathrm{M}$ etherischen $\mathrm{F}_{3} \mathrm{~B}$-Lösung tropfenweise gegeben und anschließend 12 Stunden gerührt. Anschließend wurde das Lösungsmittel im Vakuum entfernt und das Rohprodukt in $30 \mathrm{ml} n$-Hexan aufgenommen. Bei $-24{ }^{\circ} \mathrm{C}$ kristallisierten $0.53 \mathrm{~g}(0.64 \mathrm{mmol}$, $64 \%) 7$ als farbloser Feststoff aus.

\subsubsection{Darstellung von $\left[\mathrm{ClAlN}\left(2,6-i-\mathrm{Pr}_{2} \mathrm{C}_{6} \mathrm{H}_{3}\right)\right]_{3} \cdot 3 \mathrm{THF}(\mathbf{9})$ :}

$\mathrm{Zu} 0.68 \mathrm{~g}(1.04 \mathrm{mmol}) 1$ in $40 \mathrm{ml}$ THF wurden bei $0{ }^{\circ} \mathrm{C} 2.00 \mathrm{ml}(2.00 \mathrm{mmol})$ eine 1.0 $\mathrm{M} \mathrm{Cl}_{3} \mathrm{~B}$ Lösung in $n$-Hexan gegeben. Die Reaktionslösung wurde anschließend weitere 12 Stunden bei Raumtemperatur gerührt. Nach Entfernen der Lösungsmittel im Vakuum wurde das Rohprodukt in $30 \mathrm{ml}$ THF/n-Hexan (1:1) aufgenommen. Die Kristallisation erfolgte bei $26{ }^{\circ} \mathrm{C}$ über einen Zeitraum von 36 Stunden. $\left.0.89 \mathrm{~g} \mathrm{(0.96} \mathrm{mmol,} 92 \%\right) 9$ wurden als farbloser kristalliner Feststoff erhalten. 
Schmelzpunkt: $229^{\circ} \mathrm{C}$.

${ }^{1} \mathrm{H}-\mathrm{NMR}\left(250.13 \mathrm{MHz}, \mathrm{C}_{6} \mathrm{D}_{6}\right)$ :

$\delta 1.19(\mathrm{~m}, 4 \mathrm{H}, \mathrm{THF}), 1.22\left(\mathrm{~d}, J_{\mathrm{H}, \mathrm{H}}=7 \mathrm{~Hz}, 12 \mathrm{H}, \mathrm{CH}\left(\mathrm{CH}_{3}\right)_{2}\right), 1.35\left(\mathrm{~d}, J_{\mathrm{H}, \mathrm{H}}=7 \mathrm{~Hz}, 6 \mathrm{H}\right.$, $\left.\mathrm{CH}\left(\mathrm{CH}_{3}\right)_{2}\right), 1.48\left(\mathrm{~d}, J_{\mathrm{H}, \mathrm{H}}=7 \mathrm{~Hz}, 6 \mathrm{H}, \mathrm{CH}\left(\mathrm{CH}_{3}\right)_{2}\right), 1.65\left(\mathrm{~d}, J_{\mathrm{H}, \mathrm{H}}=7 \mathrm{~Hz}, 6 \mathrm{H}, \mathrm{CH}\left(\mathrm{CH}_{3}\right)_{2}\right), 1.73$ $\left(\mathrm{d}, J_{\mathrm{H}, \mathrm{H}}=7 \mathrm{~Hz}, 6 \mathrm{H}, \mathrm{CH}\left(\mathrm{CH}_{3}\right)_{2}\right), 3.30(\mathrm{~m}, 8 \mathrm{H}, \mathrm{THF}), 3.48(\mathrm{~m}, 8 \mathrm{H}, \mathrm{THF}), 3.57$ (m, $4 \mathrm{H}$, THF), 3.61 (m, $4 \mathrm{H}, \mathrm{THF}), 4.05\left(\mathrm{~m}, 4 \mathrm{H}, \mathrm{CH}\left(\mathrm{CH}_{3}\right)_{2}\right), 4.40$ (sept, $\left.\mathrm{J}_{\mathrm{H}, \mathrm{H}}=7 \mathrm{~Hz}, 2 \mathrm{H}, \mathrm{CH}\left(\mathrm{CH}_{3}\right)_{2}\right)$, $7.00(\mathrm{~m}, 9 \mathrm{H}$, arom. H) ppm.

IR (KBr, Nujol): $\widetilde{v}=3030$ (m), 1542 (m), 1422 (sst), 1358 (st), 1310 (st), 1208 (m), 1231 (sst), 1183 (sst), 1107 (st), 1039 (m), 1010 (m), 918 (m), 891 (st),861 (st), 834 (sst), 789 (sst), $746(\mathrm{~m}), 727(\mathrm{~m}), 643(\mathrm{~m}), 617(\mathrm{~m}), 546(\mathrm{st}), 445(\mathrm{~m}) \mathrm{cm}^{-1}$.

MS (EI, m/z, (\%)): 726 [M-Dipp-(i-Pr)] (5), 177 [DippNH 2$]$ (30), 162 [DippH] (100).

Elementaranalyse für $\mathrm{C}_{48} \mathrm{H}_{75} \mathrm{Al}_{3} \mathrm{Cl}_{3} \mathrm{~N}_{3} \mathrm{O}_{3}(929.43 \mathrm{~g} / \mathrm{mol})$ :

$\begin{array}{llll}\mathrm{C} & \mathrm{Cl} & \mathrm{N}\end{array}$

$\begin{array}{lllll}\text { Ber. }(\%): & 62.03 & 8.13 & 11.44 & 4.52 \\ \text { Gef. }(\%): & 61.8 & 8.0 & 11.1 & 4.5\end{array}$

5.1.5. Darstellung von $\left[\operatorname{BrAlN}\left(2,6-i-\mathrm{Pr}_{2} \mathrm{C}_{6} \mathrm{H}_{3}\right)\right]_{3} \cdot 3 \mathrm{THF}(\mathbf{1 0})$ :

$\mathrm{Zu} 0.67 \mathrm{~g}(1.03 \mathrm{mmol}) \mathbf{1}$ in $40 \mathrm{ml} \mathrm{THF}$ wurde bei $0{ }^{\circ} \mathrm{C} 3.00 \mathrm{ml}(3.00 \mathrm{mmol})$ einer 1.0 $\mathrm{M} \mathrm{Br}_{3} \mathrm{~B}$ Lösung in $n$-Hexan gegeben. Anschließend wurde die Reaktionslösung weitere 24 Stunden bei Raumtemperatur gerührt. Nach Entfernen des Lösungsmittels im Vakuum wurde das Rohprodukt in $30 \mathrm{ml} n$-Hexan aufgenommen. Die Kristallisation erfolgte bei $-4{ }^{\circ} \mathrm{C}$ über einen Zeitraum von 36 Stunden. $0.89 \mathrm{~g}$ (0.96 mmol, $92 \%) \mathbf{1 0}$ wurden als gelber kristalliner Feststoff erhalten.

Schmelzpunkt: $280{ }^{\circ} \mathrm{C}$. 
${ }^{1} \mathrm{H}-\mathrm{NMR}\left(200.13 \mathrm{MHz}, \mathrm{C}_{6} \mathrm{D}_{6}\right)$ :

$\delta 0.75(\mathrm{~m}, 4 \mathrm{H}, \mathrm{THF}), 1.32\left(\mathrm{~d}, J_{\mathrm{H}, \mathrm{H}}=7.0 \mathrm{~Hz}, 12 \mathrm{H}, \mathrm{CH}\left(\mathrm{CH}_{3}\right)_{2}\right), 1.41\left(\mathrm{~d}, J_{\mathrm{H}, \mathrm{H}}=7.0 \mathrm{~Hz}, 6 \mathrm{H}\right.$, $\left.\mathrm{CH}\left(\mathrm{CH}_{3}\right)_{2}\right), 1.49\left(\mathrm{~d}, 6 \mathrm{H}, \mathrm{CH}\left(\mathrm{CH}_{3}\right)_{2}\right), 1.61\left(\mathrm{~d}, J_{\mathrm{H}, \mathrm{H}}=7.0 \mathrm{~Hz}, 6 \mathrm{H}, \mathrm{CH}\left(\mathrm{CH}_{3}\right)_{2}\right), 3.20(\mathrm{~m}, 4 \mathrm{H}$, THF), 3.48 (m, $\left.4 \mathrm{H}, \mathrm{CH}\left(\mathrm{CH}_{3}\right)_{2}\right), 3.82\left(\mathrm{~m}, 4 \mathrm{H}, \mathrm{CH}\left(\mathrm{CH}_{3}\right)_{2}\right), 3.96$ (m, $\left.8 \mathrm{H}, \mathrm{THF}\right), 4.08$ (m, $4 \mathrm{H}$, $\left.\mathrm{CH}\left(\mathrm{CH}_{3}\right)_{2}\right), 4.37\left(\mathrm{~m}, 2 \mathrm{H}, \mathrm{CH}\left(\mathrm{CH}_{3}\right)_{2}\right), 7.10(\mathrm{~m}, 9 \mathrm{H}$, arom. $H)$ ppm.

MS (EI, $m / z,(\%)): 990$ [M-THF] (1), 177 [DippNH 2$]$ (30), 162 [DippH] (100).

Elementaranalyse für $\mathrm{C}_{48} \mathrm{H}_{75} \mathrm{Al}_{3} \mathrm{Br}_{3} \mathrm{~N}_{3} \mathrm{O}_{3}(1062.78 \mathrm{~g} / \mathrm{mol})$ :

$\begin{array}{lllll} & \mathrm{C} & \mathrm{H} & \mathrm{N} & \mathrm{Br} \\ \text { Ber.: } & 54.24 & 7.06 & 3.95 & 22.55, \\ \text { Gef.: } & 55.0 & 7.2 & 3.5 & 20.8 \% .\end{array}$

5.1.6. Umsetzung von 1 mit $\mathrm{I}_{3} \mathrm{~B}$ :

$\mathrm{Zu} 0.65 \mathrm{~g}$ (1.00 mmol) 1 in $40 \mathrm{ml}$ THF wurde bei Raumtemperatur eine Lösung von $1.17 \mathrm{~g}$ (3.00 mmol) $\mathrm{I}_{3} \mathrm{~B}$ in $30 \mathrm{ml}$ THF tropfenweise gegeben. Anschließend wurde die Reaktionslösung weitere 24 Stunden gerührt. Nach Entfernen des Lösungsmittels im Vakuum wurde das dunkle Rohprodukt in $30 \mathrm{ml} n$-Hexan aufgenommen. Der erhaltene braune Feststoff (0.61 g) konnte jedoch nicht genauer analysiert werden. Eine Variation der Reaktionstemperatur führte ebenfalls zu keinen auswertbaren Ergebnissen.

Schmelzpunkt: $>300{ }^{\circ} \mathrm{C}$

${ }^{1} \mathrm{H}-\mathrm{NMR}\left(200.13 \mathrm{MHz}, \mathrm{C}_{6} \mathrm{D}_{6}\right)$ :

$\delta 1.25$ - 7.20 ppm. (viele Signale im gesamten Bereich). 
5.1.7. Umsetzung von 1 mit $\mathrm{H}_{3} \mathrm{~B} \cdot \mathrm{THF}$, Darstellung von $\left[\mathrm{MeAlN}\left(2,6-i-\mathrm{Pr}_{2} \mathrm{C}_{6} \mathrm{H}_{3}\right)\right]_{3} \cdot 3 \mathrm{THF}$ (11):

$\mathrm{Zu} 0.72 \mathrm{~g}$ (1.10 mmol) $\mathbf{1}$ in $40 \mathrm{ml}$ THF wurden bei Raumtemperatur $3.50 \mathrm{ml}(3.50$ mmol) einer 1.0 M H $\mathrm{M}_{3} \mathrm{~B}$ Lösung in THF gegeben. Anschließend wurde die Reaktionslösung weitere 24 Stunden bei Raumtemperatur gerührt. Nach Entfernen des Lösungsmittels im Vakuum wurde das Rohprodukt in $30 \mathrm{ml} n$-Hexan aufgenommen. Die Kristallisation erfolgte bei $-26{ }^{\circ} \mathrm{C}$ über einen Zeitraum von 24 Stunden. $0.54 \mathrm{~g}$ (0.96 mmol, $\left.92 \%\right) \mathbf{1 1}$ wurden als farbloser kristalliner Feststoff erhalten.

Schmelzpunkt: $210^{\circ} \mathrm{C}$.

${ }^{1} \mathrm{H}-\mathrm{NMR}\left(500.13 \mathrm{MHz}, \mathrm{C}_{6} \mathrm{D}_{6}\right)$ :

$\delta$-0.85 (s, $\left.9 \mathrm{H}, \mathrm{AlCH}_{3}\right), 1.29\left(\mathrm{~d}, J_{\mathrm{H}, \mathrm{H}}=6.9 \mathrm{~Hz}, 36 \mathrm{H}, \mathrm{CH}\left(\mathrm{CH}_{3}\right)_{2}\right), 1.32\left(\mathrm{~m}, 14 \mathrm{H}, \mathrm{OCH}_{2} \mathrm{CH}_{2}\right)$, $3.67\left(\mathrm{~m}, 10 \mathrm{H}, \mathrm{OCH}_{2} \mathrm{CH}_{2}\right), 3.90$ (sept, $\left.J_{\mathrm{H}, \mathrm{H}}=6.9 \mathrm{~Hz}, 6 \mathrm{H}, \mathrm{CH}\left(\mathrm{CH}_{3}\right)_{2}\right), 7.10$ (m, $9 \mathrm{H}$, arom. $H$ ) ppm.

MS (EI, m/z, (\%)): 651 [M-THF] (35), 177 [DippN] (45), 162 [DippH] (100).

5.1.8. Umsetzung von 1 mit $\mathrm{PhSi}(\mathrm{C} \equiv \mathrm{CH})_{3}$ :

$\mathrm{Zu} 1.19 \mathrm{~g}$ (1.83 mmol) 1 in $40 \mathrm{ml}$ Toluol wurde bei $-78{ }^{\circ} \mathrm{C}$ eine Lösung aus $0.33 \mathrm{~g}$ (1.83 mmol) $\mathrm{PhSi}(\mathrm{C} \equiv \mathrm{CH})_{3}$ in $30 \mathrm{ml}$ Toluol tropfenweise zugegeben. Nach Aufarbeitung wurde das Rohprodukt mit $30 \mathrm{ml} n$-Pentan gewaschen und filtriert. Der erhaltene braune Feststoff konnte nicht genauer analysiert werden.

Schmelzpunkt: $250^{\circ} \mathrm{C}$ (Zersetzung).

${ }^{1} \mathrm{H}-\mathrm{NMR}\left(250.23 \mathrm{MHz}, \mathrm{C}_{6} \mathrm{D}_{6}\right)$ :

$\delta-0.80-8.20$ ppm (viele Resonanzen im gesamten Bereich). 


\subsection{Darstellung und Hydrolyse von monoorganylsubstituierten Germaniumtrihalogeniden}

5.2.1. Darstellung von 2,6-Bis(2`,6`-dimethylphenyl)iodbenzol (12):

$34.0 \mathrm{~g}$ (125 mmol) 2,6-Dichloriodbenzol wurden in $200 \mathrm{ml}$ THF gelöst, dazu tropfte man innerhalb von 90 Minuten $250 \mathrm{mmol}$ einer etherischen Grignard-Lösung aus 2,6Dimethylphenylmagnesiumbromid. Nachdem die Reaktionslösung sechs Stunden unter Rückfluss kochte, wurde mit $130 \mathrm{~g}(0.52 \mathrm{~mol}) \mathrm{I}_{2}$ in $400 \mathrm{ml}$ THF bei $0{ }^{\circ} \mathrm{C}$ gequencht. Überschüssiges $\mathrm{I}_{2}$ wurde mit $500 \mathrm{ml}$ einer gesättigten $\mathrm{Na}_{2} \mathrm{~S}_{2} \mathrm{O}_{5}$-Lösung zerstört. Daraufhin separierte man die Phasen und schüttelte die wässrige dreimal mit $200 \mathrm{ml}$ Ether aus. Die organische Phase wurde mit $\mathrm{MgSO}_{4}$ getrocknet und auf $200 \mathrm{ml}$ eingeengt. Bei $-26{ }^{\circ} \mathrm{C}$ kristallisierten 23.2 g (56.2 mmol, $45 \%) \mathbf{1 2}$ als schwachgelber Feststoff aus.

Schmelzpunkt: $151-153{ }^{\circ} \mathrm{C}$.

${ }^{1} \mathrm{H}-\mathrm{NMR}\left(200.13 \mathrm{MHz}, \mathrm{C}_{6} \mathrm{D}_{6}\right)$ :

$\delta 2.04$ (s, $12 \mathrm{H}, \mathrm{CH}), 6.77$ (m, $2 \mathrm{H}$, arom. H), 7.08 (m, 7 H, arom. H) ppm.

${ }^{13} \mathrm{C}-\mathrm{NMR}\left(50.32 \mathrm{MHz}, \mathrm{C}_{6} \mathrm{D}_{6}\right)$ :

$\delta 20.51\left(\mathrm{CH}_{3}\right), 127.77$ (arom. C), 127.81 (arom. C), 128.29 (arom. C), 129.13 (arom. C), 135.56 (arom. C), 145.12 (arom. C), 147.78 (arom. C) ppm.

IR (KBr, Nujol): $\widetilde{v}=2909$ (m), 1578 (st), 1448 (sst), 1385 (st), 1272 (st), 1162 (m), 1078 (sst), 1012 (sst), 1000 (st), 804 (sst), 772 (sst) $\mathrm{cm}^{-1}$.

MS (EI, m/z, (\%)): 412 [M] (35), 397 [M-Me] (100).

Elementaranalyse für $\mathrm{C}_{22} \mathrm{H}_{21} \mathrm{I}(412.29 \mathrm{~g} / \mathrm{mol})$ :

$\mathrm{C}$ $\mathrm{H}$ I

Ber.:

64.09

30.78 ,

Gef.:

63.8

$29.8 \%$. 
5.2.2. Darstellung von 2,6-Bis(2‘,6`-dimethylphenyl)phenylgermaniumtrichlorid (13):

$0.76 \mathrm{ml}$ (1.90 mmol) einer 2.5 M $n$-BuLi Lösung in Hexan wurden bei $-78^{\circ} \mathrm{C}$ zu einer Lösung von $0.79 \mathrm{~g}$ (1.91 mmol) 2,6-Bis(2‘,6'-dimethylphenyl)iodbenzol in $50 \mathrm{ml} n$-Hexan tropfenweise zugesetzt. Anschließend rührte die Reaktionslösung 12 Stunden bei Raumtemperatur. Nach Entfernen aller flüchtigen Bestandteile im Vakuum und Lösen des Lithiumsalzes in $30 \mathrm{ml} \mathrm{Et} 2 \mathrm{O}$ wurde bei $-78{ }^{\circ} \mathrm{C} 0.23 \mathrm{ml}(0.42 \mathrm{~g}, 2.00 \mathrm{mmol}) \mathrm{Cl}_{4} \mathrm{Ge}_{\text {gegeben, }}$ wobei $\mathrm{LiCl}$ als farbloser Feststoff ausfiel. Nach 18 Stunden Reaktionszeit bei Raumtemperatur und anschließender Filtration wurde das Lösungsmittel im Vakuum entfernt. Das Rohprodukt wurde in $10 \mathrm{ml}$ THF/n-Hexan (1:1) aufgenommen. Bei Raumtemperatur kristallisierten $0.75 \mathrm{~g}(1.60 \mathrm{mmol}, 84 \%) \mathbf{1 3}$ als farbloser Feststoff aus.

Schmelzpunkt: $179-181^{\circ} \mathrm{C}$.

${ }^{1} \mathrm{H}-\mathrm{NMR}\left(500.26 \mathrm{MHz}, \mathrm{C}_{6} \mathrm{D}_{6}\right)$ :

$\delta 2.03\left(\mathrm{~s}, 6 \mathrm{H}, \mathrm{CH}_{3}\right), 2.06\left(\mathrm{~s}, 6 \mathrm{H}, \mathrm{CH}_{3}\right), 6.67-7.05$ (m, $9 \mathrm{H}$, arom. $H$ ) ppm.

${ }^{13} \mathrm{C}-\mathrm{NMR}\left(125.77 \mathrm{MHz}, \mathrm{C}_{6} \mathrm{D}_{6}\right)$ :

$\delta 20.34\left(\mathrm{CH}_{3}\right), 20.98\left(\mathrm{CH}_{3}\right), 128.12$ (arom. C), 130.65 (arom. C), 133.40 (arom. C), 135.99 (arom. C), 137.10 (arom. C), 139.14 (arom. C), 145.13 (arom. C), 147.81 (arom. C) ppm.

IR (KBr, Nujol): $\tilde{v}=3063$ (m), 3020 (m), 2854 (st), 1938 (m), 1863 (m), 1581 (st), 1399 (st), 1262 (sst), 1163 (st), 1093 (sst), 1031 (sst), 876 (m), 798 (sst), 769 (sst), 741 (st), 696 (st), 409 (st) $\mathrm{cm}^{-1}$.

MS (EI, m/z, (\%)): 464 [M] (30), 429 [M-Cl] (4), 357 [M-3 Cl] (4), 284 [Ar] (100).

Elementaranalyse für $\mathrm{C}_{22} \mathrm{H}_{21} \mathrm{Cl}_{3} \mathrm{Ge}(464.33 \mathrm{~g} / \mathrm{mol})$ :

$\mathrm{C}$

Ber. (\%):

56.90

57.4
$\mathrm{H}$

4.56

4.9
$\mathrm{Cl}$

22.90

22.9 
5.2.3. Darstellung von 2,6-Bis(2‘,6`-dimethylphenyl)phenylgermaniumtribromid (14):

$\mathrm{Zu} 0.80 \mathrm{~g}$ (1.53 mmol) 2,6-Bis(2‘,6‘-dimethylphenyl)iodbenzol in $30 \mathrm{ml} n$-Hexan wurden bei $-78{ }^{\circ} \mathrm{C} 0.61 \mathrm{ml}(1.53 \mathrm{mmol})$ eine $2.5 \mathrm{M} n$-BuLi Lösung in Hexan getropft. Anschließend rührte die Reaktionslösung 12 Stunden bei Raumtemperatur. Nach Entfernen aller flüchtigen Bestandteile im Vakuum und Lösen des Lithiumsalzes wurden bei $-78{ }^{\circ} \mathrm{C} 0.60$ $\mathrm{g}(1.53 \mathrm{mmol}) \mathrm{Br}_{4} \mathrm{Ge}$ in $20 \mathrm{ml} \mathrm{Et}{ }_{2} \mathrm{O}$ gegeben, danach rührte die Reaktionslösung weitere 18 Stunden bei Raumtemperatur. Das entstandene $\mathrm{BrLi}$ wurde abfiltriert und flüchtige Bestandteile im Vakuum entfernt. Das Rohprodukt wurde in $10 \mathrm{ml} n$-Hexan aufgenommen. Bei $0{ }^{\circ} \mathrm{C}$ kristallisierten $0.68 \mathrm{~g}$ (1.14 mmol, $\left.74 \%\right) \mathbf{1 4}$ in Form quaderförmiger gelblicher Kristalle aus.

Schmelzpunkt: $191-194^{\circ} \mathrm{C}$.

${ }^{1} \mathrm{H}-\mathrm{NMR}\left(500.26 \mathrm{MHz}, \mathrm{C}_{6} \mathrm{D}_{6}\right)$ :

$\delta 2.05\left(\mathrm{~s}, 6 \mathrm{H}, \mathrm{CH}_{3}\right), 2.08\left(\mathrm{~s}, 6 \mathrm{H}, \mathrm{CH}_{3}\right), 6.66-7.10(\mathrm{~m}, 9 \mathrm{H}$, arom. H) ppm.

${ }^{13} \mathrm{C}-\mathrm{NMR}\left(125.77 \mathrm{MHz}, \mathrm{C}_{6} \mathrm{D}_{6}\right)$ :

$\delta 20.48\left(\mathrm{CH}_{3}\right), 21.84\left(\mathrm{CH}_{3}\right), 129.08$ (arom. C), 129.09 (arom. C), 130.65 (arom. C), 131.15 (arom. C), 135.80 (arom. C), 137.43 (arom. C), 141.76 (arom. C), 143.37 (arom. C) ppm.

IR (KBr, Nujol): $\widetilde{v}=3047$ (st), 3002 (st), 2831 (st), 1918 (m), 1841 (m), 1532 (st), 1374 (sst), 1232 (st), 1172 (st), 1103 (sst), 1001 (sst), 856 (m), 772 (sst), 747 (sst), 719 (st), 674 (st), $384(\mathrm{st}) \mathrm{cm}^{-1}$.

MS (EI, $m / z,(\%)): 598$ [M] (4), 517 [M-Br] (12), 285 [ArH] (4), 153 [Ar-4 Me-Ph] (100).

Elementaranalyse für $\mathrm{C}_{22} \mathrm{H}_{21} \mathrm{Br}_{3} \mathrm{Ge}(597.71 \mathrm{~g} / \mathrm{mol})$ :

$\mathrm{C}$

Ber.: 44.21 45.0
$\mathrm{H}$ 3.54 3.9
$\mathrm{Br}$

40.10, $39.8 \%$. 
5.2.4. Darstellung von 2,6-Bis(2‘,6'-dimethylphenyl)phenylgermaniumtriiodid (15):

$0.64 \mathrm{ml}$ einer 2.5 $\mathrm{M} n$-BuLi Lösung in Hexan wurden bei $-78^{\circ} \mathrm{C}$ zu einer Lösung von $0.65 \mathrm{~g}$ (1.00 mmol) 2,6-Bis(2`,6'-dimethylphenyl)iodbenzol in $40 \mathrm{ml} n$-Hexan tropfenweise zugegeben. Anschließend rührte die Reaktionslösung 12 Stunden bei Raumtemperatur. Nachdem alle flüchtigen Bestandteile im Vakuum entfernt wurden, löste man das Lithiumsalz in $30 \mathrm{ml} \mathrm{Et} t_{2} \mathrm{O}$. $\mathrm{Zu}$ dieser Lösung wurden bei $-78^{\circ} \mathrm{C} 0.58 \mathrm{~g}, 1.00 \mathrm{mmol}$ ) $\mathrm{I}_{4} \mathrm{Ge}$ in $30 \mathrm{ml}$ Toluol gegeben. Nach 18 Stunden Reaktionszeit bei Raumtemperatur wurde das Lösungsmittel im Vakuum entfernt. Das mit ILi verunreinigte Rohprodukt wurde dann mit $40 \mathrm{ml} n$-Hexan aufgenommen und anschließend filtriert. Bei $-26^{\circ} \mathrm{C}$ kristallisierten $0.34 \mathrm{~g}(0.46 \mathrm{mmol}, 46 \%)$ $\mathbf{1 5}$ als gelber Feststoff aus.

Schmelzpunkt: $143^{\circ} \mathrm{C}$.

${ }^{1} \mathrm{H}-\mathrm{NMR}\left(200.13 \mathrm{MHz}, \mathrm{C}_{6} \mathrm{D}_{6}\right)$ :

$\delta 2.03\left(\mathrm{~s}, 12 \mathrm{H}, \mathrm{CH}_{3}\right), 6.74-7.18(\mathrm{~m}, 9 \mathrm{H}$, arom. $H)$ ppm.

MS (EI, m/z, (\%)): 739 [M] (12), 612 [M-I] (8), 454 [I 3 Ge] (100), 285 [Ar] (4).

Elementaranalyse für $\mathrm{C}_{22} \mathrm{H}_{21} \mathrm{GeI}_{3}(738.73 \mathrm{~g} / \mathrm{mol})$ :

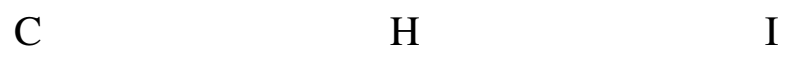

$\begin{array}{llll}\text { Ber.: } & 35.72 & 2.86 & 51.46, \\ \text { Gef.: } & 34.1 & 2.7 & 50.9 \% .\end{array}$


5.2.5. Darstellung von 2,6-Bis(2‘,6'-dimethylphenyl)phenylgermaniumtrifluorid (16):

$1.38 \mathrm{~g}$ (3.00 mmol) 2,6-Bis(2‘,6'-dimethylphenyl)phenylgermaniumtrichlorid und $1.62 \mathrm{~g} \mathrm{(3.00} \mathrm{mmol)} \mathrm{Me}_{3} \mathrm{SnF}$ in $50 \mathrm{ml}$ Toluol wurden bei Raumtemperatur 24 Stunden gerührt, wobei alle unlöslichen Komponenten abreagierten. Anschließend wurden alle flüchtigen Bestandteile im Vakuum entfernt. Das Rohprodukt konnte durch fraktionierte Destillation (Sdp.: $120^{\circ} \mathrm{C} / 0.2$ Torr) gereinigt werden. Es wurden $0.32 \mathrm{~g}(0.77 \mathrm{mmol}, 26 \%) 16$ erhalten.

${ }^{1} \mathrm{H}-\mathrm{NMR}\left(200.13 \mathrm{MHz}, \mathrm{C}_{6} \mathrm{D}_{6}\right)$ :

$\delta 2.01\left(\mathrm{~m}, 12 \mathrm{H}, \mathrm{CH}_{3}\right), 6.83-7.08(\mathrm{~m}, 9 \mathrm{H}$, arom. $H)$ ppm.

${ }^{19}$ F-NMR (188.29 MHz, $\left.\mathrm{C}_{6} \mathrm{D}_{6}\right)$ :

$\delta-42.83 \mathrm{ppm}$.

MS (EI, $m / z,(\%)): 415$ [M] (8), 358 [GeAr] (32), 285 [Ar] (100).

Elementaranalyse für $\mathrm{C}_{22} \mathrm{H}_{21} \mathrm{~F}_{3} \mathrm{Ge}(415.01 \mathrm{~g} / \mathrm{mol})$ :<smiles>[1H]</smiles>

Ber.: $\quad 63.61 \quad 5.06$,

Gef.: $\quad 63.1 \quad 4.9 \%$.

5.2.6. Darstellung von $\operatorname{DippNSiMe}_{3} \mathrm{GeF}_{3}$ (17):

Analog zu 5.2.5. wurden $1.68 \mathrm{~g}$ (3.00 mmol) DippNSiMe $\mathrm{GeBr}_{3}$ und $1.62 \mathrm{~g}$ (3.00 mmol) $\mathrm{Me}_{3} \mathrm{SnF}$ in $50 \mathrm{ml} \mathrm{THF}$ vorgelegt. Es wurden $0.55 \mathrm{~g}$ (1.47 mmol, 49 \%) 17 (Sdp.: 143 ${ }^{\circ} \mathrm{C} / 0.2$ Torr) erhalten.

${ }^{1} \mathrm{H}-\mathrm{NMR}\left(200.13 \mathrm{MHz}, \mathrm{C}_{6} \mathrm{D}_{6}\right)$ :

$\delta 0.18\left(\mathrm{~m}, 9 \mathrm{H}, \mathrm{SiCH}_{3}\right), 1.14\left(\mathrm{~m}, 12 \mathrm{H}, \mathrm{CHCH}_{3}\right), 3.49$ (sept, $\left.2 \mathrm{H}, \mathrm{CHCH}_{3}\right), 7.00$ (m, $3 \mathrm{H}$, arom. $H$ ) ppm. 
${ }^{19}$ F-NMR (188.29 MHz, $\left.\mathrm{C}_{6} \mathrm{D}_{6}\right)$ :

$\delta-39.79 \mathrm{ppm}$.

MS (EI, m/z, (\%)): 375 [M] (4), 248 [DippNSiMe $]$ (100).

Elementaranalyse für $\mathrm{C}_{15} \mathrm{H}_{23} \mathrm{~F}_{3} \mathrm{GeNSi}(375.04 \mathrm{~g} / \mathrm{mol})$ :<smiles>[1H]</smiles>

Ber.: $\quad 48.04 \quad 6.18$,

Gef.: $\quad 48.1 \quad 5.9 \%$.

\subsection{Versuche zur Hydrolyse von Monoorganylgermaniumtrihalogeniden}

5.3.1. Umsetzung von $\operatorname{DippN}\left(\mathrm{SiMe}_{3}\right) \mathrm{GeBr}_{3}$ mit $\mathrm{PhNH}_{2}$ und $\mathrm{H}_{2} \mathrm{O}$ :

$\mathrm{Zu} 0.99 \mathrm{~g}$ (1.76 mmol) $\mathrm{DippN}\left(\mathrm{SiMe}_{3}\right) \mathrm{GeBr}_{3}$ in $50 \mathrm{ml} \mathrm{Et}{ }_{2} \mathrm{O}$ wurden bei $-78{ }^{\circ} \mathrm{C} 10 \mu \mathrm{l}$ (0.10 g, $5.47 \mathrm{mmol}) \mathrm{H}_{2} \mathrm{O}$ und $0.49 \mathrm{~g}(5.47 \mathrm{mmol})$ Anilin in $50 \mathrm{ml} \mathrm{Et}_{2} \mathrm{O}$ gegeben, wobei sich ein farbloser Niederschlag von $\mathrm{PhNH}_{2} \mathrm{HBr}$ bildete. Nachdem die Reaktionslösung 24 Stunden bei Raumtemperatur rührte, wurde der Niederschlag abgetrennt und das Lösungsmittel des Filtrats im Vakuum entfernt. Es zeigte sich, dass eventuell entstandenes Produkt mit dem Aniliniumbromid ausgefallen war. Versuche, diese Verbindungen voneinander zu trennen, schlugen fehl.

${ }^{1} \mathrm{H}-\mathrm{NMR}\left(200.13 \mathrm{MHz}, \mathrm{CD}_{3} \mathrm{CN}\right)$ :

$\delta 0.03$ (s, $\left.9 \mathrm{H}, \mathrm{SiCH}_{3}\right), 1.02\left(\mathrm{~m}, 12 \mathrm{H}, \mathrm{CHCH}_{3}\right), 3.45$ (m, $2 \mathrm{H}, \mathrm{CHCH}_{3}$ ), 4.20 (br. s, $3 \mathrm{H}, \mathrm{NH}_{3}$ ), $6.50-6.99$ (m, 8 H, arom. $H$ ) ppm.

${ }^{29} \mathrm{Si}-\mathrm{NMR}\left(99.36 \mathrm{MHz}, \mathrm{CD}_{3} \mathrm{CN}\right)$ :

$\delta 8.93$ (SiMe) ppm. 
5.3.2. Umsetzung von $\operatorname{DippN}\left(\mathrm{SiMe}_{3}\right) \mathrm{GeBr}_{3}$ mit Pyridin und $\mathrm{H}_{2} \mathrm{O}$, Darstellung von $\left[\left(\mathrm{DippNSiMe} \mathrm{Ge}_{4} \mathrm{O}_{6}\right](\mathbf{1 8})\right.$ :

$\mathrm{Zu} 0.94 \mathrm{~g}$ (1.68 mmol) DippN($\left(\mathrm{SiMe}_{3}\right) \mathrm{GeBr}_{3}$ in $30 \mathrm{ml}$ Toluol wurde eine Lösung aus 91.6 $\mu \mathrm{l}(91.6 \mathrm{mg}, 5.09 \mathrm{mmol}) \mathrm{H}_{2} \mathrm{O}$ und $0.40 \mathrm{~g}$ (5.06 mmol) Pyridin in $40 \mathrm{ml}$ Toluol bei $-78{ }^{\circ} \mathrm{C}$ getropft. Anschließend rührte das Gemisch für 24 Stunden bei Raumtemperatur. Danach wurde filtriert und das Lösungsmittel im Vakuum entfernt. Das Rohprodukt wurde dann in 20 $\mathrm{ml} n$-Hexan aufgenommen. Nach 36 Stunden bei $-26^{\circ} \mathrm{C}$ kristallisierten $0.36 \mathrm{~g}$ (0.26 mmol, 62 \%) $\mathbf{1 8}$ als quaderförmige Kristalle aus.

Schmelzpunkt: $214-218{ }^{\circ} \mathrm{C}$.

${ }^{1} \mathrm{H}-\mathrm{NMR}\left(200.13 \mathrm{MHz}, \mathrm{C}_{6} \mathrm{D}_{6}\right)$ :

$\delta 0.33\left(\mathrm{~s}, 9 \mathrm{H}, \mathrm{SiCH}_{3}\right), 1.29\left(\mathrm{~m}, 12 \mathrm{H}, \mathrm{CHCH}_{3}\right), 3.78\left(\mathrm{sept},{ }^{3} \mathrm{~J}_{\mathrm{H}, \mathrm{H}}=7.0 \mathrm{~Hz}, 2 \mathrm{H}, \mathrm{CHCH}_{3}\right), 7.06$ (m, $3 \mathrm{H}$, arom. $H$ ) ppm.

${ }^{29} \mathrm{Si}-\mathrm{NMR}\left(99.36 \mathrm{MHz}, \mathrm{C}_{6} \mathrm{D}_{6}\right)$ :

$\delta 10.88(\mathrm{SiMe}) \mathrm{ppm}$.

IR (KBr, Nujol): $\widetilde{v}=3613$ (st), 3515 (m), 3056 (m), 1438 (st), 1382 (st), 1260 (sst), 1178 (st), 1101 (st), 1019 (st), 929 (m), 800 (sst), 718 (m), 533 (st), 438 (m) cm . $^{-1}$

MS (EI, m/z, (\%)): 1087 [M-DippNSiMe $3-3 \mathrm{Me}$ ] (4), 1074 [M-DippNSiMe 3 -(i-Pr)-Me] (4), 248 [DippNSiMe $]$ (86), 73 [SiMe 3$](100)$.

Elementaranalyse für $\mathrm{C}_{60} \mathrm{H}_{104} \mathrm{Ge}_{4} \mathrm{~N}_{4} \mathrm{O}_{6} \mathrm{Si}_{4}(1380.27 \mathrm{~g} / \mathrm{mol})$ :

$\begin{array}{llllll} & \mathrm{C} & \mathrm{H} & \mathrm{N} & \mathrm{Ge} & \mathrm{Si} \\ \text { Ber.: } & 52.21 & 7.59 & 4.06 & 21.04 & 8.14, \\ \text { Gef.: } & 51.5 & 7.8 & 3.8 & 19.9 & 7.9 \% .\end{array}$


5.3.3. Umsetzung von $\operatorname{DippN}\left(\mathrm{SiMe}_{3}\right) \mathrm{GeBr}_{3}$ mit $\mathrm{H}_{2} \mathrm{O}_{2}$ :

$\mathrm{Zu} \quad 2.30 \mathrm{~g}(4.10 \mathrm{mmol}) \quad \mathrm{DippN}\left(\mathrm{SiMe}_{3}\right) \mathrm{GeBr}_{3}$ in $40 \mathrm{ml}$ Toluol wurden bei Raumtemperatur $5 \mathrm{ml}$ einer $15 \%$ igen $\mathrm{H}_{2} \mathrm{O}_{2}$ Lösung gegeben. Nachdem die Reaktionslösung 12 Stunden rührte, wurde die Lösung für weitere 12 Stunden unter siedendem Toluol gerührt. Nach Aufarbeitung wurde das Ausgangsprodukt quantitativ zurückerhalten. Wiederholungen des Experimentes mit unterschiedlichen Reaktionszeiten ergaben ebenfalls das Edukt.

\subsubsection{Umsetzung von $\left[\mathrm{DippN}\left(\mathrm{SiMe}_{3}\right) \mathrm{Ge}\left(\mathrm{NH}_{2}\right)_{2}\right]_{2} \mathrm{NH}$ mit $\mathrm{H}_{2} \mathrm{O}$ :}

$\mathrm{Zu} 0.37 \mathrm{~g}(0.51 \mathrm{mmol})\left[\mathrm{DippN}\left(\mathrm{SiMe}_{3}\right) \mathrm{Ge}\left(\mathrm{NH}_{2}\right)_{2}\right]_{2} \mathrm{NH}$ in $40 \mathrm{ml} \mathrm{Et}_{2} \mathrm{O}$ wurden bei -78 ${ }^{\circ} \mathrm{C} 111 \mu \mathrm{l}(0.11 \mathrm{~g}, 6 \mathrm{mmol}) \mathrm{H}_{2} \mathrm{O}$ tropfenweise gegeben. Die Reaktionsmischung wurde weitere drei Stunden bei dieser Temperatur und anschließend drei Stunden bei Raumtemperatur gerührt. Danach wurden alle flüchtigen Bestandteile im Vakuum entfernt und das Rohprodukt in $20 \mathrm{ml}$ Pentan aufgenommen. Bei $0{ }^{\circ} \mathrm{C}$ kristallisierten $0.15 \mathrm{~g}(0.11$ mmol, $21 \%) \mathbf{1 8}$ aus.

Die spektroskopischen Daten $\left({ }^{1} \mathrm{H},{ }^{13} \mathrm{C}-\mathrm{NMR}\right)$ zeigten, dass es sich um eine Mischung verschiedener Produkte handelte, wobei $\mathbf{1 8}$ als Teil dieser Mischung identifiziert werden konnte.

MS (EI, m/z, (\%)): 1380 [M] (4), 1043 [M-DippNSiMe 3 -Pr-3Me] (40), 248 [DippNSiMe 3 ] (76), $73\left[\mathrm{SiMe}_{3}\right](100)$.

IR (KBr, Nujol): $\widetilde{v}=3616$ (st), 1735 (m), 1463 (sst), 1438 (sst), 1382 (st), 1265 (st), 1180 (st), 1092 (st), 1043 (st), 918 (st), 839 (sst), 798 (sst), 755 (m), 696 (st), 535 (st) cm-1. 
5.3.5. Umsetzung von $\mathrm{DippNSiMe}_{3} \mathrm{GeBr}_{3}$ mit $\mathrm{KOH}$ im Zwei-Phasensystem Ammoniak/Toluol:

$\mathrm{Zu} 1.07 \mathrm{~g}$ (1.91 mmol) DippNSiMe $\mathrm{GeBr}_{3}$ und $0.29 \mathrm{~g}$ (5.61 mmol) gemörsertem $\mathrm{KOH}$ in $30 \mathrm{ml}$ Toluol wurden bei $-78{ }^{\circ} \mathrm{C} 30 \mathrm{ml}$ über $\mathrm{Na}$ getrockneter Ammoniak einkondensiert. Anschließend wurde das Zweiphasensystem für 30 Minuten bei dieser Temperatur gerührt, danach wurde die Temperatur schrittweise auf Raumtemperatur gebracht, wobei überschüssiger Ammoniak verdampfte. Nach Filtration von $\mathrm{KCl}$ wurde das Lösungsmittel im Vakuum entfernt. Es wurde ein farbloser Feststoff $(0.45 \mathrm{~g})$ erhalten, der jedoch nicht als einheitliches Produkt identifiziert werden konnte. Im Massenspektrum wird u.a. der Molekülionenpeak von 18 gefunden.

${ }^{1} \mathrm{H}-\mathrm{NMR}\left(200.16 \mathrm{MHz}, \mathrm{C}_{6} \mathrm{D}_{6}\right)$ :

$\delta 0.32\left(\mathrm{~m}, \mathrm{SiCH}_{3}\right), 1.23$ (m, $\mathrm{CHCH}_{3}$ ), 2.00 (br s, OH), 2.25 (br s, OH), 2.64 (sept, ${ }^{3} J=7.0 \mathrm{~Hz}$, $\mathrm{CHCH}_{3}$ ), 3.18 (br s, $\mathrm{OH}$ ), 3.67 (sept, ${ }^{3} \mathrm{~J}=7.0 \mathrm{~Hz}, \mathrm{CHCH}_{3}$ ), $3.78\left(\mathrm{~m}, \mathrm{CHCH}_{3}\right.$ ), 7.06 (m, arom. H) ppm.

${ }^{29} \mathrm{Si}-\mathrm{NMR}\left(500 \mathrm{MHz}, \mathrm{C}_{6} \mathrm{D}_{6}\right)$ :

mehrere Resonanzen im Bereich zwischen $\delta 9.23$ bis 12.05 ppm.

IR (KBr, Nujol): $\widetilde{v}=3614$ (st), 3516 (m), 3055 (m), 1438 (sst), 1382 (st), 1363 (m), 1253 (sst), 1179 (st), 1103 (st), 1019 (st), 930 (m), 839 (st), 799 (sst), 718 (m), 533 (st), 438 (m) $\mathrm{cm}^{-1}$.

MS (EI, m/z, (\%)): 1380 [M] (12), 1074 [M-DippNSiMe 3 -Pr-Me] (68), 248 [DippNSiMe $]$ (100). 
5.3.6. Umsetzung von 2,6-Bis(2‘,6`-dimethylphenyl)phenylgermaniumtrichlorid mit $\mathrm{KOH} / \mathrm{NH}_{3}$, Darstellung von $\left[\left(\mathrm{R}^{*} \mathrm{Ge}\right)_{4} \mathrm{O}_{6}\right](\mathbf{1 9})$ :

Zu 1.25 g (2.69 mmol) 2,6-Bis(2‘,6'-dimethylphenyl)phenylgermaniumtrichlorid 0.44 $\mathrm{g}(7.90 \mathrm{mmol}) \mathrm{KOH}$ und $40 \mathrm{ml} \mathrm{Et} 2 \mathrm{O}$ wurden bei $-78{ }^{\circ} \mathrm{C} 30 \mathrm{ml}$ getrockneter Ammoniak einkondensiert. Daraufhin rührte die Reaktionslösung für 30 Minuten bei dieser Temperatur. Nach Auftauen auf Raumtemperatur, Filtration vom Feststoff und Entfernen des Lösungsmittels im Vakuum wurden $1.23 \mathrm{~g}(0.80 \mathrm{mmol}) 19$ als farbloser Feststoff erhalten.

Schmelzpunkt: $162^{\circ} \mathrm{C}$

${ }^{1} \mathrm{H}-\mathrm{NMR}\left(200.13 \mathrm{MHz}, \mathrm{CDCl}_{3}\right)$ :

$\delta 2.03\left(\mathrm{~s}, 12 \mathrm{H}, \mathrm{CH}_{3}\right), 7.17(\mathrm{~m}, 9 \mathrm{H}$, arom. $H)$ ppm.

${ }^{13} \mathrm{C}-\mathrm{NMR}\left(100.60 \mathrm{MHz}, \mathrm{CDCl}_{3}\right)$ :

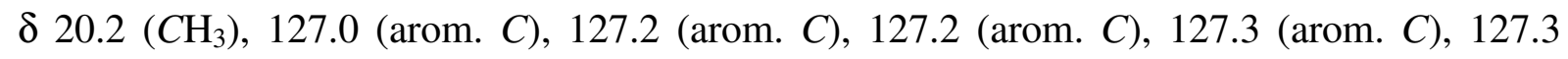
(arom. C), 127.6 (arom. C), 129.4 (arom. C), 136.0 (arom. C), 139.5 (arom. C), 140.5 (arom. C) ppm.

IR (KBr, Nujol): $\widetilde{v}=3618$ (m), 1399 (st), 1378 (sst), 1260 (m), 1165 (m), 1115 (st), 1078 (st), 1042 (st), 801 (st), 768 (sst), 743 (st), 715 (m) $\mathrm{cm}^{-1}$.

MS (EI, m/z, (\%)): 1243 [M-R ] (4), 1156 [M-R $\left.{ }^{*} \mathrm{Ge}-\mathrm{Me}\right]$ (4), 373 [R $\left.{ }^{*} \mathrm{GeO}\right](16), 357$ [R* Ge] (24), $285\left[\mathrm{R}^{*}\right]$ (52), $269\left[\mathrm{Ar}^{*}-\mathrm{Me}\right](100)$.

Elementaranalyse für $\mathrm{C}_{88} \mathrm{H}_{84} \mathrm{Ge}_{4} \mathrm{O}_{6}(1528.04 \mathrm{~g} / \mathrm{mol})$ :

$\begin{array}{llll} & \mathrm{C} & \mathrm{H} & \mathrm{Ge} \\ \text { Ber.: } & 73.81 & 5.91 & 20.27, \\ \text { Gef.: } & 74.1 & 6.1 & 19.2 \% .\end{array}$




\subsection{Umsetzungen von Verbindungen mit niedervalenten Elementen der 14. Gruppe}

5.4.1. Darstellung von (Dipp) ${ }_{2} \mathrm{NacNacAlHBH}_{2}(\mathbf{2 0})$ :

$\mathrm{Zu} \quad 0.55 \mathrm{~g}(1.09 \mathrm{mmol})(\mathrm{Dipp})_{2} \mathrm{NacNacGeH} \cdot \mathrm{BH}_{3}$ in $50 \mathrm{ml}$ Toluol wurden bei Raumtemperatur $3 \mathrm{ml}(1.20 \mathrm{mmol})$ einer $0.40 \mathrm{M}$ Lösung $\mathrm{H}_{3} \mathrm{Al} \cdot \mathrm{NMe}_{3}$ in Toluol gegeben. Nachdem die Reaktionslösung drei Stunden unter Rückfluss kochte, wurden alle flüchtigen Bestandteile im Vakuum entfernt. Der Rückstand wurde in $10 \mathrm{ml} n$-Hexan aufgenommen. Bei $-26{ }^{\circ} \mathrm{C}$ kristallisierten $0.42 \mathrm{~g}(0.92 \mathrm{mmol}) \mathbf{2 0}$ in Form hell gelber Kristalle aus.

Schmelzpunkt: $132{ }^{\circ} \mathrm{C}$.

${ }^{1} \mathrm{H}-\mathrm{NMR}\left(200.13 \mathrm{MHz}, \mathrm{C}_{6} \mathrm{D}_{6}\right)$ :

$\delta 1.13\left(\mathrm{~d}, J_{\mathrm{H}, \mathrm{H}}=7.0 \mathrm{~Hz}, 6 \mathrm{H}, \mathrm{CH}\left(\mathrm{CH}_{3}\right)_{2}\right), 1.38\left(\mathrm{~d}, J_{\mathrm{H}, \mathrm{H}}=7.0 \mathrm{~Hz}, 6 \mathrm{H}, \mathrm{CH}\left(\mathrm{CH}_{3}\right)_{2}\right), 1.54(\mathrm{~s}, 6$ $\left.\mathrm{H}, \mathrm{CH}_{3}\right), 3.41$ (sept, $\left.J_{\mathrm{H}, \mathrm{H}}=7.0 \mathrm{~Hz}, 4 \mathrm{H}, \mathrm{CH}\left(\mathrm{CH}_{3}\right)_{2}\right), 4.85(\mathrm{~s}, 1 \mathrm{H}, \mathrm{CH}), 7.11(\mathrm{~m}, 6 \mathrm{H}$, arom. $H)$ ppm.

${ }^{11} \mathrm{~B}-\mathrm{NMR}\left(96.23 \mathrm{MHz}, \mathrm{C}_{6} \mathrm{D}_{6}\right)$ :

$\delta-7.5 \mathrm{ppm}$.

${ }^{13} \mathrm{C}-\mathrm{NMR}\left(100.60 \mathrm{MHz}, \mathrm{CDCl}_{3}\right)$ :

$\delta 22.8\left(\mathrm{NC}\left(\mathrm{CH}_{3}\right) \mathrm{CH}\right), 24.0\left(\mathrm{CH}\left(\mathrm{CH}_{3}\right)_{2}\right), 24.8\left(\mathrm{CH}\left(\mathrm{CH}_{3}\right)_{2}\right), 28.4\left(\mathrm{CH}\left(\mathrm{CH}_{3}\right)_{2}\right), 94.3(\gamma-\mathrm{C}), 123.1$ (arom. C), 125.1 (arom. C), 141.2 (arom. C), 150.2 (arom. C), 163.3 (arom. C) ppm.

IR (KBr, Nujol): $\widetilde{v}=3059$ (m), 2726 (m), 2500 (st), 1832 (m), 1796 (st), 1533 (sst), 1441 (sst), 1384 (st), 1260 (sst), 1177 (m), 1096 (sst), 1019 (sst), 936 (m), 874 (m), 799 (sst), 722 (m), $678(\mathrm{~m}) \mathrm{cm}^{-1}$.

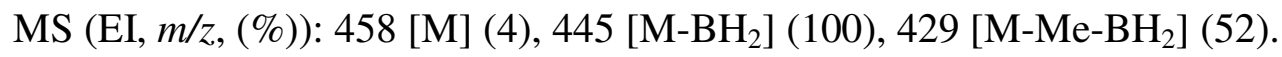


Elementaranalyse für $\mathrm{C}_{29} \mathrm{H}_{44} \mathrm{AlBN}_{2}(458.48 \mathrm{~g} / \mathrm{mol})$ :

$\begin{array}{lllll} & \mathrm{C} & \mathrm{H} & \mathrm{B} & \mathrm{N} \\ \text { Ber. (\%): } & 75.97 & 9.67 & 2.36 & 6.11, \\ \text { Gef. (\%): } & 74.8 & 8.9 & 2.1 & 5.9 \% .\end{array}$

5.4.2. Versuch zur Darstellung von (Dipp) $)_{2} \mathrm{NacNacSnF:}$

$0.58 \mathrm{~g}(1.01 \mathrm{mmol})(\mathrm{Dipp})_{2} \mathrm{NacNacSnCl}$ und $0.20 \mathrm{~g}(1.09 \mathrm{mmol}) \mathrm{Me}_{3} \mathrm{SnF}$ in $50 \mathrm{ml}$ $\mathrm{CH}_{2} \mathrm{Cl}_{2}$ wurden für zwei Tage unter Rückfluss gekocht. Nach Aufarbeitung wurden quantitativ die Edukte zurückgewonnen. Variationen von Stöchiometrie und Reaktionsbedingungen schlugen ebenso fehl.

Alternativ: $\mathrm{Zu}$ einer Suspension aus $0.42 \mathrm{~g}$ (2.60 mmol) $\mathrm{F}_{2} \mathrm{Sn}$ und $40 \mathrm{ml}$ THF wurden bei Raumtemperatur $1.14 \mathrm{~g}(2.60 \mathrm{mmol})$ (Dipp) ${ }_{2} \mathrm{NacNacLi}$ in $20 \mathrm{ml} \mathrm{THF}$ tropfenweise gegeben. Anschließend rührte die Suspension drei Tage im siedenden THF. Nach Aufarbeitung waren die Edukte isolierbar.

\subsubsection{Darstellung von $(t-\mathrm{BuSn})_{4} \mathrm{~S}_{6}(\mathbf{2 1})$ :}

Eine Suspension von $0.89 \mathrm{~g}(1.50 \mathrm{mmol})(\mathrm{Dipp})_{2} \mathrm{NacNacSn}(t-\mathrm{Bu})$ und $48.1 \mathrm{mg}(1.50$ mmol) Schwefel in $30 \mathrm{ml}$ Toluol wurde für drei Stunden unter Rückfluss erhitzt. Nach Filtration und Entfernen aller flüchtigen Bestandteile im Vakuum wurde das Rohprodukt in $30 \mathrm{ml} n$-Hexan aufgenommen. Nach drei Tagen bei $-26{ }^{\circ} \mathrm{C}$ kristallisierten $0.23 \mathrm{~g}(0.25 \mathrm{mmol}$, $66 \%) 21$ aus.

Schmelzpunkt: $210{ }^{\circ} \mathrm{C}$.

${ }^{1} \mathrm{H}-\mathrm{NMR}\left(200.16 \mathrm{MHz}, \mathrm{C}_{6} \mathrm{D}_{6}\right)$ :

$\delta 1.29\left(\mathrm{CH}_{3}\right) \mathrm{ppm}$. 
${ }^{13} \mathrm{C}-\mathrm{NMR}\left(125.77 \mathrm{MHz}, \mathrm{C}_{6} \mathrm{D}_{6}\right)$ :

$\delta 28.2\left(\mathrm{C}\left(\mathrm{CH}_{3}\right)_{3}\right), 42.9\left(\mathrm{C}\left(\mathrm{CH}_{3}\right)_{3}\right) \mathrm{ppm}$.

${ }^{119} \mathrm{Sn}-\mathrm{NMR}\left(186.50 \mathrm{MHz}, \mathrm{C}_{6} \mathrm{D}_{6}\right)$ :

$\delta$ - $243.7 \mathrm{ppm}$.

MS (EI, $m / z,(\%)): 838$ [M-Bu] (80), 782 [M-2Bu] (24), 636 [Sn $\left.\mathrm{S}_{5}\right]$ (100).

Elementaranalyse für $\mathrm{C}_{16} \mathrm{H}_{36} \mathrm{~S}_{6} \mathrm{Sn}_{4}(895.69 \mathrm{~g} / \mathrm{mol})$ :

$\begin{array}{lll}\mathrm{C} & \mathrm{H} & \mathrm{S}\end{array}$

$\begin{array}{llll}\text { Ber.: } & 21.46 & 4.05 & 21.48, \\ \text { Gef.: } & 21.0 & 3.8 & 20.9 \% .\end{array}$




\subsection{Behandlung und Entsorgung der Abfälle}

Lösungsmittel wurden abdestilliert oder in Kühlfallen aufgefangen und getrennt nach halogenhaltigen und halogenfreien Abfällen in die bereitgestellten Sammelbehälter gegeben. Handelte es sich nicht um Gemische und konnte davon ausgegangen werden, daß keine reaktiven Reaktionsrückstände mitgerissen worden waren, wurden die Lösungsmittel getrennt gesammelt und redestilliert. Deuterierte Lösungsmittel aus NMR-Messungen wurden je nach Halogengehalt wie beschrieben entsorgt.

Abfälle, die bei der Synthese von Liganden anfielen, wurden vorsichtig hydrolysiert und in die wässrigen Säure-/Base-Abfälle gegeben. Reaktionsrückstände wurden vorsichtig mit Ethanol oder feuchtem Aceton hydrolysiert und in die bereitgestellten SchwermetallSammelbehälter gegeben.

Bei Trocknungen anfallende Natriumreste wurden gesammelt und regeneriert, ${ }^{[145]}$ bevor sie erneut eingesetzt wurden. Reste, die nicht mehr aufbereitet werden konnten, wurden mit Ethanol hydrolysiert und entweder in das alkalische Reinigungsbad für Glasgeräte gegeben oder neutralisiert und dem kommunalen Abwassernetz zugeführt. ${ }^{[146]}$

Andere Trockenmittelreste wie $\mathrm{KOH}, \mathrm{P}_{4} \mathrm{O}_{10}$ und $\mathrm{CaH}_{2}$ überführte man nach langsamer vollständiger Hydrolyse an der Raumluft in die Säure- bzw. Basenabfälle.

Aceton aus Kühlbädern wurde nach Entweichen des $\mathrm{CO}_{2}$ zur Reinigung der Glasgeräte verwendet. 
Die bei der Arbeit angefallenen Mengen an Sonderabfall können wie folgt abgeschätzt werden:

Halogenhaltige Lösungsmittel:

Halogenfreie Lösungsmittel:

501

Saure Abfälle:

201

Alkalische Abfälle:

301

Schwermetallhaltige Abfälle: 


\section{Röntgenographischer Anhang}

Verbindung

Summenformel

Molmasse

Temperatur

Wellenlänge

Kristallsystem

Raumgruppe

Zelldimension

Zellvolumen

Formeleinheit pro Zelle

Absorptionskoeffizient

$\mathrm{F}(000)$

Gemessener $\theta$-Bereich

Indexgrenzen

Anzahl der gemessenen Reflexe

Unabhängige Reflexe

Strukturverfeinerung

Daten/Restraints/Parameter

Goodness-of-Fit an $F^{2}$

Endgültige $R$-Werte $[I>2 \sigma(I)]$

$R$-Werte (sämtliche Daten)

Größtes Maximum und Minimum
7

$\mathrm{C}_{48} \mathrm{H}_{75} \mathrm{Al}_{3} \mathrm{~F}_{3} \mathrm{~N}_{3} \mathrm{O}_{3}$

$880.05 \mathrm{~g} / \mathrm{mol}$

200(2) K

$0.71073 \AA$

monoklin

$P 2{ }_{1} / n$

$a=12.258(4) \AA$

$b=21.069(7) \AA$

$c=19.035(9) \AA$

$\beta=93.45(3)^{\circ}$

491(3) $\AA^{3}$

4

$0.130 \mathrm{~mm}^{-1}$

1896

$3.53-25.05^{\circ}$

$-14 \leq h \leq 14$

$-1 \leq k \leq 25$

$-22 \leq l \leq 22$

9295

$8666\left(\mathrm{R}_{\text {int }}=0.0511\right)$

Vollmatrix Least-Squares an $F^{2}$

8666 / 0 / 553

1.040

$R 1=0.0536, w R 2=0.1204$

$R 1=0.0765, w R 2=0.1393$

0.386 und $-0.288 \mathrm{e} \cdot \AA^{-3}$. 
Verbindung

Summenformel

Molmasse

Temperatur

Wellenlänge

Kristallsystem

Raumgruppe

Zelldimension

Zellvolumen

Formeleinheit pro Zelle

Absorptionskoeffizient

$\mathrm{F}(000)$

Gemessener $\theta$-Bereich

Indexgrenzen

Anzahl der gemessenen Reflexe

Unabhängige Reflexe

Strukturverfeinerung

Daten/Restraints/Parameter

Goodness-of-Fit an $F^{2}$

Endgültige $R$-Werte $[I>2 \sigma(I)]$

$R$-Werte (sämtliche Daten)

Größtes Maximum und Minimum
8

$\mathrm{C}_{28} \mathrm{H}_{46} \mathrm{~N}_{2} \mathrm{Sn}_{2}$

$648.08 \mathrm{~g} / \mathrm{mol}$

133(2) K

$0.71073 \AA$

monoklin

$P 2_{1} / n$

$a=9.849(2) \AA$

$b=8.9882(18) \AA$

$c=16.879(3) \AA$

$\beta=100.35(3)^{\circ}$

$1469.8(5) \AA^{3}$

2

$1.716 \mathrm{~mm}^{-1}$

656

$3.18-24.67^{\circ}$

$-11 \leq h \leq 11$

$-10 \leq k \leq 10$

$-19 \leq l \leq 19$

8205

$2352\left(\mathrm{R}_{\text {int }}=0.0888\right)$

Vollmatrix Least-Squares an $F^{2}$

2352 / 0 / 151

1.130

$R 1=0.0252, w R 2=0.0747$

$R 1=0.0262, w R 2=0.0756$

0.478 und $-0.565 \mathrm{e} \cdot \AA^{-3}$. 
Verbindung

Summenformel

Molmasse

Temperatur

Wellenlänge

Kristallsystem

Raumgruppe

Zelldimension

Zellvolumen

Formeleinheit pro Zelle

Absorptionskoeffizient

$\mathrm{F}(000)$

Gemessener $\theta$-Bereich

Indexgrenzen

Anzahl der gemessenen Reflexe

Unabhängige Reflexe

Strukturverfeinerung

Daten/Restraints/Parameter

Goodness-of-Fit an $F^{2}$

Endgültige $R$-Werte $[I>2 \sigma(I)]$

$R$-Werte (sämtliche Daten)

Größtes Maximum und Minimum

\section{2}

$\mathrm{C}_{22} \mathrm{H}_{21} \mathrm{I}$

$412.29 \mathrm{~g} / \mathrm{mol}$

203(2) K

$0.71073 \AA$

orthorhombisch

Pbca

$a=8.5762(8) \AA$

$b=13.7456(17) \AA$

$c=31.867(19) \AA$

$\beta=90^{\circ}$

376(1) $\AA^{3}$

8

$1.703 \mathrm{~mm}^{-1}$

1648

$3.53-25.05^{\circ}$

$-10 \leq h \leq 3$

$-16 \leq k \leq 16$

$-37 \leq l \leq 0$

3338

$3315\left(\mathrm{R}_{\mathrm{int}}=0.0151\right)$

Vollmatrix Least-Squares an $F^{2}$

3315 / 0 / 212

1.086

$R 1=0.0260, w R 2=0.0627$

$R 1=0.0316, w R 2=0.0672$

0.302 und $-0.612 \mathrm{e} \cdot \AA^{-3}$. 
Verbindung

Summenformel

Molmasse

Temperatur

Wellenlänge

Kristallsystem

Raumgruppe

Zelldimension

Zellvolumen

Formeleinheit pro Zelle

Absorptionskoeffizient

$\mathrm{F}(000)$

Gemessener $\theta$-Bereich

Indexgrenzen

Anzahl der gemessenen Reflexe

Unabhängige Reflexe

Strukturverfeinerung

Daten/Restraints/Parameter

Goodness-of-Fit an $F^{2}$

Endgültige $R$-Werte $[I>2 \sigma(I)]$

$R$-Werte (sämtliche Daten)

Größtes Maximum und Minimum

\section{3}

$\mathrm{C}_{22} \mathrm{H}_{21} \mathrm{Cl}_{3} \mathrm{Ge}$

$464.33 \mathrm{~g} / \mathrm{mol}$

153(2) K

$0.71073 \AA$

orthorhobisch

Pbca

$a=17.858(5) \AA$

$b=12.489(3) \AA$

$c=19.346(9) \AA$

$\beta=90^{\circ}$

432(1) $\AA^{3}$

8

$1.795 \mathrm{~mm}^{-1}$

1888

$3.51-25.01^{\circ}$

$0 \leq h \leq 21$

$-14 \leq k \leq 11$

$-22 \leq l \leq 0$

8980

$3774\left(\mathrm{R}_{\mathrm{int}}=0.0576\right)$

Vollmatrix Least-Squares an $F^{2}$

3774 / 0 / 239

1.066

$R 1=0.0498, w R 2=0.1163$

$R 1=0.0746, w R 2=0.1324$

0.682 und $-0.536 \mathrm{e} \cdot \AA^{-3}$. 
Verbindung

Summenformel

Molmasse

Temperatur

Wellenlänge

Kristallsystem

Raumgruppe

Zelldimension

Zellvolumen

Formeleinheit pro Zelle

Absorptionskoeffizient

$\mathrm{F}(000)$

Gemessener $\theta$-Bereich

Indexgrenzen

Anzahl der gemessenen Reflexe

Unabhängige Reflexe

Strukturverfeinerung

Daten/Restraints/Parameter

Goodness-of-Fit an $F^{2}$

Endgültige $R$-Werte [ $I>2 \sigma(I)]$

$R$-Werte (sämtliche Daten)

Größtes Maximum und Minimum

\section{4}

$\mathrm{C}_{22} \mathrm{H}_{21} \mathrm{Br}_{3} \mathrm{Ge}$

$597.71 \mathrm{~g} / \mathrm{mol}$

203(2) K

$0.71073 \AA$

orthorhombisch

Pbca

$a=17.816(4) \AA$

$b=12.885(3) \AA$

$c=19.248(4) \AA$

$\beta=90^{\circ}$

441.87(15) $\AA^{3}$

8

$6.816 \mathrm{~mm}^{-1}$

2320

$3.53-22.52^{\circ}$

$-19 \leq h \leq 19$

$-6 \leq k \leq 13$

$-20 \leq l \leq 20$

4391

$2806\left(R_{\text {int }}=0.1395\right)$

Vollmatrix Least-Squares an $F^{2}$

2806 / 0 / 239

0.993

$R 1=0.1034, w R 2=0.2456$

$R 1=0.1398, w R 2=0.2882$

2.411 und $-2.136 \mathrm{e} \cdot \AA^{-3}$. 
Verbindung

Summenformel

Molmasse

Temperatur

Wellenlänge

Kristallsystem

Raumgruppe

Zelldimension

Zellvolumen

Formeleinheit pro Zelle

Absorptionskoeffizient

$\mathrm{F}(000)$

Gemessener $\theta$-Bereich

Indexgrenzen

Anzahl der gemessenen Reflexe

Unabhängige Reflexe

Strukturverfeinerung

Daten/Restraints/Parameter

Goodness-of-Fit an $F^{2}$

Endgültige $R$-Werte [ $I>2 \sigma(I)]$

$R$-Werte (sämtliche Daten)

Größtes Maximum und Minimum
20

$\mathrm{C}_{29} \mathrm{H}_{44} \mathrm{AlBN}_{2}$

$458.45 \mathrm{~g} / \mathrm{mol}$

200(2) K

$0.71073 \AA$

monoklin

$\mathrm{P} 21 / n$

$a=12.544(2) \AA$

$b=16.337(4) \AA$

$c=14.066(3) \AA$

$\beta=104.941(14)^{\circ}$

278.52(10) $\AA^{3}$

4

$0.091 \mathrm{~mm}^{-1}$

1000

$1.94-24.83^{\circ}$

$-14 \leq h \leq 14$

$-19 \leq k \leq 19$

$-16 \leq l \leq 16$

16464

$4755\left(\mathrm{R}_{\mathrm{int}}=0.0953\right)$

Vollmatrix Least-Squares an $F^{2}$

4755 / 1 / 307

0.945

$R 1=0.0650, w R 2=0.1680$

$R 1=0.0919, w R 2=0.1796$

1.005 und $-0.407 \mathrm{e} \cdot \AA^{-3}$. 


\section{Literaturverzeichnis}

[1] N. N. Greenwood, A. Earnshaw, Chemie der Elemente, 1. Aufl., VCH, Weinheim, 1988.

[2] R. T. Sanderson, Polar Covalence, Academic Press, New York, 1983.

[3] A. F. Holleman, E. Wiberg, Lehrbuch der Anorganischen Chemie, 101. Aufl., de Gruyter, Berlin, 1995.

[4] M. Witt, H. W. Roesky, Curr. Sci. 2000, 78, 410-430 und dort zitierte Literatur.

[5] K. Ziegler, R. Köster, Ann. Chem. 1957, 608, 1-7.

[6] G. Hamprecht, M. Tittel, Dt. Pat. 1961, 1.102.151; Chem. Abstr. 1962, 56, $6.000 \mathrm{a}$.

[7] H. Lehmkuhl, O. Olbrysch, H. Nehl, Ann. Chem. 1973, 708-714.

[8] K. Ziegler, Brit. Pat. 1957, 779.873; Chem. Abstr. 1957, 51, 17.981 b.

[9] G. Hamprecht, M. Tittel, Dt. Pat. 1962, 1.116.660; Chem. Abstr. 1962, 56, $10.189 \mathrm{~b}$.

[10] S. H. Eidt, Dt. Pat. 1972, 2.151.176; Chem. Abstr. 1972, 77, 19.793x.

[11] H. Jenkner, Z. Naturforsch. 1957, B12, 809.

[12] M. R. Kopp, T. Kräuter, B. Werner, B. Neumüller, Z. Anorg. Allg. Chem. 1998, 624, 881-886.

[13] R. G. Bulgakov, V. N. Yakovlev, G. Y. Maistrenko, G. A. Tolstikov, V. P. Kazakov, Izv. Akad. Nauk, SSR Ser. Khim. 1986, 490; Bull. Acad. Sci. USSR Div. Chem. Sci. 1986, 453.

[14] G. A. Atiya, A. S. Grady, S. A. Jackson, N. Parker, D. K. Russell, J. Organomet. Chem. 1989, 378, 307-316.

[15] B. Neumüller, Coord. Chem. Rev. 1997, 158, 69-101.

[16] H. W. Roesky, Inorg. Chem. 1999, 38, 5934-5943.

[17] A. Herzog, F.-Q. Liu, H. W. Roesky, A. Demsar, K. Keller, M. Noltemeyer, F. Pauer, Organometallics 1994, 13, 1251-1256.

[18] H. W. Roesky, A. Herzog, K. Keller, Z. Naturforsch. 1994, B49, 981-982. 
[19] H. W. Roesky, A. Herzog, F.-Q. Liu, J. Fluorine Chem. 1995, 72, 183-185.

[20] M. G. Walawalkar, R. Murugavel, H. W. Roesky, Eur. J. Solid State Inorg. Chem. 1996, 33, 943-955.

[21] F. Jäger, H. W. Roesky, H. Dorn, S. Shah, M. Noltemeyer, H.-G. Schmidt, Chem. Ber. 1997, 130, 399-403.

[22] S. D. Waezsada, F.-Q. Liu, E. F. Murphy, H. W. Roesky, M. Teichert, I. Usón, H.-G. Schmidt, T. Albers, E. Parisini, M. Noltemeyer, Organometallics 1997, $16,1260-1264$.

[23] C. Schnitter, K. Klimek, H. W. Roesky, T. Albers, H.-G. Schmidt, C. Röpken, E. Parisini, Organometallics 1998, 17, 2249-2257.

[24] S. D. Waezsada, F.-Q. Liu, C. E. Barnes, H. W. Roesky, M. L. Montero, I. Usón, Angew. Chem. 1997, 109, 2738-2739; Angew. Chem. Int. Ed. Engl. 1997, 36, 2625-2626.

[25] R. G. Pearson, J. Am. Chem. Soc. 1963, 85, 3533-3539.

[26] R. G. Pearson, Inorg. Chem. 1988, 27, 734-740.

[27] H. Hatop, H. W. Roesky, T. Labahn, C. Röpken, G. M. Sheldrick, M. Bhattacharjee, Organometallics 1998, 17, 4326-4328.

[28] H. Hatop, H. W. Roesky, T. Labahn, A. Fischer, H.-G. Schmidt, M. Noltemeyer, Organometallics 2000, 19, 937-940.

[29] H.-H. Brintzinger, D. Fischer, R. Mülhaupt, B. Rieger, R. Waymouth, Angew. Chem. 1995, 107, 1255-1282; Angew. Chem. Int. Ed. Engl. 1995, 34, 1143-1170.

[30] Y.-X. Chen, M. V. Metz, L. Li, C. L. Stern, T. J. Marks, J. Am. Chem. Soc. 1998, 120, 6287-6305.

[31] C. Rennekamp, H. Wessel, H. W. Roesky, P. Müller, H.-G. Schmidt, M. Noltemeyer, I. Usón, A. R. Barron, Inorg. Chem. 1999, 38, 5235-5240.

[32] H. Wessel, H.-S. Park, P. Müller, H. W. Roesky, I. Usón, Angew. Chem. 1999, 111, 850-852; Angew. Chem. Int. Ed. 1999, 38, 813-815.

[33] B. Twamley, S. T. Haubrich, P. P. Power, Adv. Organomet. Chem. 1999, 44, 1-65 und dort zitierte Literatur.

[34] P. P. Power, Chem. Rev. 1999, 99, 3463-3503. 
[35] N. Tokitoh, Y. Arai, T. Sasamori, R. Okazaki, S. Nagase, H. Uekusa, Y. Ohashi, J. Am. Chem. Soc. 1998, 120, 433-434.

[36] N. Tokitoh, Y. Arai, R. Okazaki, S. Nagase, Science 1997, 277, 78-80.

[37] N. Tokitoh, R. Okazaki, Adv. Organomet. Chem. 2001, 47, 121-166.

[38] B. Twamley, C. D. Sofield, M. M. Olmstead, P. P. Power, J. Am. Chem. Soc. 1999, $121,3357-3367$.

[39] L. Pu, B. Twamley, P. P. Power, J. Am. Chem. Soc. 2000, 122, 3524-3525.

[40] A. D. Phillips, R. J. Wright, M. M. Olmstead, P. P. Power, J. Am. Chem. Soc. 2002, 124, 5930-5931.

[41] M. Stender, A. D. Phillips, R. J. Wright, P. P. Power, Angew. Chem. 2002, 114, 1863-1865; Angew. Chem. Int. Ed. 2002, 41, 1785-1787.

[42] K. W. Klinkhammer, Angew. Chem. 1997, 109, 2414; Angew. Chem. Int. Ed. Engl. 1997, 36, 2320-2322.

[43] P. P. Power, J. Chem. Soc., Dalton Trans. 1998, 2939-2951.

[44] G. H. Robinson, Acc. Chem. Res. 1999, 32, 773-782.

[45] A. J. Downs, Coord. Chem. Rev. 1999, 189, 59-100.

[46] H. Grützmacher, T. Fässler, Chem. Eur. J. 2000, 6, 2317-2325.

[47] (a) Y. Ding, H. W. Roesky, M. Noltemeyer, H.-G. Schmidt, P. P. Power, Organometallics 2001, 20, 1190-1194; (b) Y. Ding, H. Hao, H. W. Roesky, M. Noltemeyer, H.-G. Schmidt, Organometallics 2001, 20, 4806-4811.

[48] J. Prust, A. Stasch, W. Zheng, H. W. Roesky, E. Alexopoulos, I. Usón, D. Böhler, T. Schuchardt, Organometallics 2001, 20, 3825-3828.

[49] J. Feldman, S. J. McLain, A. Parthasaraty, W. J. Marshall, J. C. Calabrese, S. D. Arthur, Organometallics 1997, 16, 1514-1516.

[50] V. C. Gibson, P. J. Maddox, C. Newton, C. Redshaw, G. A. Solan, A. J. P. White, D. J. Williams, Chem. Commun. 1998, 1651-1652.

[51] V. C. Gibson, J. A. Segal, A. J. P. White, D. J. Williams, J. Am. Chem. Soc. 2000, 122, 7120-7121.

[52] C. E. Radzewich, M. P. Coles, R. F. Jordan, J. Am. Chem. Soc. 1998, 120, 9384-9385.

[53] N. Kuhn, J. Fahl, S. Fuchs, M. Steinmann, Z. Anorg. Allg. Chem. 1999, 625, 
2108-2114.

[54] B. Qian, D. L. Ward, M. R. Smith, III, Organometallics 1998, 17, 3070-3076.

[55] J. Prust, H. Hohmeister, A. Stasch, H. W. Roesky, J. Magull, E. Alexopoulos, I. Usón, H.-G. Schmidt, M. Noltemeyer, Eur. J. Inorg. Chem. 2002, 21562162.

[56] S. T. Haubrich, P. P. Power, J. Am. Chem. Soc. 1998, 120, 2202-2203.

[57] C. Cui, H. W. Roesky, H.-G. Schmidt, M. Noltemeyer, H. Hao, F. Cimpoesu Angew. Chem. 2000, 112, 4444-4446; Angew. Chem. Int. Ed. 2000, 39, 42744276.

[58] J. Prust, K. Most, I. Müller, E. Alexopoulos, A. Stasch, I. Usón, H. W. Roesky, Z. Anorg. Allg. Chem. 2001, 627, 2032-2037.

[59] N. J. Hardman, C. Cui, H. W. Roesky, W. H. Fink, P. P. Power, Angew. Chem. 2001, 113, 2230-2232; Angew. Chem. Int. Ed. 2001, 40, 2172-2174.

[60] Y. Ding, Q. Ma, I. Usón, H. W. Roesky, M. Noltemeyer, H.-G. Schmidt, J. Am. Chem. Soc. 2002, 124, 8542-8543.

[61] H. N. Stokes, Chem. Ber. 1891, 24, 933-936.

[62] A. Ladenburg, Chem Ber. 1871, 4, 91-94.

[63] A. Ladenburg, Ann. Chem. Pharm. 1872, 164, 300-302.

[64] R. Murugavel, A. Voigt, M. G. Walawalkar, H. W. Roesky, Chem. Rev. 1996, 96, 2205-2236.

[65] M. Shakir, H. W. Roesky, Phosphorus, Sulfur, Silicon 1994, 93, 13-38.

[66] R. Murugavel, V. Chandrasekhar, H. W. Roesky, Acc. Chem. Res. 1996, 29, 183-189.

[67] F. J. Feher, T. A. Budzichowski, Polyhedron 1995, 14, 3239-3253.

[68] R. Murugavel, V. Chandrasekhar, A. Voigt, H. W. Roesky, H.-G. Schmidt, M. Noltemeyer, Organometallics 1995, 14, 5298-5301.

[69] N. Winkhofer, A. Voigt, H. Dorn, H. W. Roesky, A. Steiner, D. Stalke, A. Reller, Angew. Chem. 1994, 106, 1414-1416; Angew. Chem. Int. Ed. Engl. 1994, 33, 1352-1354.

[70] M. L. Montero, I. Usón, H. W. Roesky, Angew. Chem. 1994, 106, 2198-2199; Angew. Chem. Int. Ed. Engl. 1994, 33, 2103-2104. 
[71] M. L. Montero, A. Voigt, M. Teichert, I. Usón, H. W. Roesky, Angew. Chem. 1995, 107, 2761-2763; Angew. Chem. Int. Ed. Engl. 1995, 34, 2504-2506.

[72] V. Chandrasekhar, R. Murugavel, A. Voigt, H. W. Roesky, H.-G. Schmidt, Organometallics 1996, 15, 918-922.

[73] P. Jutzi, H. Schmidt, B. Neumann, H.-G. Stammler, Organometallics 1996, $15,741-746$.

[74] H. Puff, S. Franken, W. Schuh, W. Schwab, J. Organomet. Chem. 1983, 254, $33-41$.

[75] G. Ferguson, J. F. Gallagher, D. Murphy, T. R. Spalding, C. Glidewell, H. D. Holden, Acta Crystallogr. 1992, C48, 1228-1231.

[76] C. Glidewell, M. B. Hursthouse, D. Lloyd, K. W. Lumbard, R. L. Short, J. Chem. Res. 1986, 400-401.

[77] A. Kawachi, Y. Tanaka, K. Tamao, Organometallics 1997, 16, 5102-5107.

[78] J. Beckmann, K. Jurkschat, M. Schürmann, Eur. J. Inorg. Chem. 2000, 939941.

[79] V. Lorenz, K. Jacob, C. Wagner, H. Görls, Z. Anorg. Allg. Chem. 2002, 628, 2855-2861.

[80] H. Puff, K. Braun, S. Franken, T. R. Kok, W. Schuh, J. Organomet. Chem. 1988, 349, 293-303.

[81] L. Pu, N. J. Hardman, P. P. Power, Organometallics 2001, 20, 5105-5109.

[82] D. A. Armitage, The Chemistry of Organic Silicon Compounds, John Wiley \& Sons, London, 1989.

[83] H. Yoshida, Y. Kabe, W. Ando, Organometallics 1991, 10, 27-29 und dort zitierte Literatur.

[84] M. Unno, Y. Kawai, H. Shioyama, H. Matsumoto, Organometallics 1997, 16, 4428-4434.

[85] W. Ando, T. Kadowaki, Y. Kabe, M. Ishii, Angew. Chem. 1992, 104, 84-85; Angew. Chem. Int. Ed. Engl. 1992, 31, 59-60.

[86] K. Wraage, L. Lameyer, D. Stalke, H. W. Roesky, Eur. J. Inorg. Chem. 1999, 869-872.

[87] K. M. Waggoner, H. Hope, P. P. Power, Angew. Chem. 1988, 100, 1765-1766; 
Angew. Chem. Int. Ed. Engl. 1988, 27, 1699-1700.

[88] B. M. Gimarc, Pure Appl. Chem. 1990, 60, 423-428.

[89] H. Wessel, C. Rennekamp, H. W. Roesky, M. L. Montero, P. Müller, I. Usón, Organometallics 1998, 17, 1919-1921.

[90] H. Wessel, M. L. Montero, C. Rennekamp, H. W. Roesky, P. Yu, I. Usón, Angew. Chem. 1998, 110, 862-864; Angew. Chem. Int. Ed. 1998, 37, 843-845.

[91] H. Wessel, Dissertation, Göttingen 1999.

[92] H. Hohmeister, H. Wessel, P. Lobinger, H. W. Roesky, P. Müller, I. Usón, H.G. Schmidt, M. Noltemeyer, J. Magull, J. Fluorine Chem. 2003, 120, 59-64.

[93] G. Shanmugasunderan, G. Nagarajan, Z. Phys. Chem. 1969, 240, 363-370.

[94] I. Krossing, H. Nöth, C. Tacke, M. Schmidt, H. Schwenk, Chem. Ber./Recueil 1997, 130, 1047-1052.

[95] M. A. Petrie, P. P. Power, H. V. R. Dias, K. Ruhlandt-Senge, K. M. Waggoner, R. J. Wehmschulte, Organometallics 1993, 12, 1086-1093.

[96] R. J. Wehmschulte, W. J. Grigsby, B. Schiemenz, R. A. Bartlett, P. P. Power, Inorg. Chem. 1996, 35, 6694-6702.

[97] H. Puff, D. Hanssgen, N. Beckermann, A. Roloff, W. Schuh, J. Organomet. Chem. 1989, 373, 37-47.

[98] S. Fukuzumi, S. Kuroda, T. Tanaka, J. Chem. Soc., Perkin Trans. 2 1986, 2529.

[99] H. Grützmacher, H. Pritzkow, Angew. Chem. 1991, 103, 976; Angew. Chem. Int. Ed. Engl. 1991, 30, 1017-1018.

[100] H. W. Roesky, A. Stasch, H. Hatop, C. Rennekamp, D. H. Hamilton, M. Noltemeyer, H.-G. Schmidt, Angew. Chem. 2000, 112, 177-179; Angew. Chem. Int. Ed. 2000, 39, 121-123.

[101] K. Ziegler, E. Holzkamp, R. Köster, H. Lehmkuhl, Angew. Chem. 1955, 67, 213-214.

[102] B. R. Jagirdar, E. F. Murphy, H. W. Roesky, Prog. Inorg. Chem. 1999, 48, 351-455.

[103] B. Neumüller, F. Gahlmann, Chem. Ber. 1993, 126, 1579-1585.

[104] M. R. Kopp, B. Neumüller, Z. Anorg. Allg. Chem. 1998, 624, 1393-1394. 
[105] D. Chakraborty, S. Horchler, H. W. Roesky, M. Noltemeyer, H.-G. Schmidt, Inorg. Chem. 2000, 39, 3995-3998.

[106] W. Uhl, F. Breher, B. Neumüller, Organometallics 2001, 20, 5478-5484.

[107] M. Schiefer, Dissertation, Göttingen 2002.

[108] W. J. Zheng, H. Hohmeister, N. C. Mösch-Zanetti, H. W. Roesky, M. Noltemeyer, H.-G. Schmidt, Inorg. Chem. 2001, 40, 2363-2367.

[109] A. Stasch, M. Ferbinteanu, J. Prust, W. Zheng, F. Cimpoesu, H. W. Roesky, J. Magull, H.-G. Schmidt, M. Noltemeyer, J. Am. Chem. Soc. 2002, 124, 54415448 .

[110] H. Wessel, mündliche Mitteilung

[111] H. Wessel, C. Rennekamp, S. D. Waezsada, H. W. Roesky, M. L. Montero, I. Usón, Organometallics 1997, 16, 3243-3245.

[112] A. Meller, Fortschr. Chem. Forsch. 1970, 15, 146-190.

[113] J. Pinkas, J. Löbl, D. Dastych, M. Necas, H. W. Roesky, Inorg. Chem. 2002, 41, 6914-6918.

[114] U. Krüerke, J. Organomet. Chem. 1970, 21, 83-90.

[115] B. Räke, mündliche Mitteilung

[116] A. Saednya, H. Hart, Synthesis 1996, 1455-1458.

[117] C. Manthey, Dissertation, Kiel, 1998.

[118] M. Niemeyer, Organometallics 1998, 17, 4649-4656.

[119] B. Twamley, N. J. Hardman, P. P. Power, Acta Crystallogr. 2000, C56, 514515.

[120] M. Unno, Y. Kawai, H. Matsumoto, Heteroat. Chem. 2001, 12, 238-243.

[121] G. M. Apalkova, A. I. Gusev, S. N. Gurkova, A. E. Feoktistov, Metallorg. Khim. 1988, 1, 942; Organomet. Chem. USSR.

[122] G. S. Zaitseva, S. S. Karlov, A. V. Churakov, J. A. K. Howard, E. V. Avtomonov, J. Lorberth, Z. Anorg. Allg. Chem. 1997, 623, 1144-1150.

[123] P. T. Matsunaga, J. Kouvetakis, T. L. Groy, Inorg. Chem. 1995, 34, 51035104.

[124] H. Hao, H. W. Roesky, Y. Ding, C. Cui, M. Schormann, H.-G. Schmidt, M. Noltemeyer, B. Zemva, J. Fluorine Chem. 2002, 115, 143-147. 
[125] S. Richelme, M. Andrianarison, C. Couret, J. Escudie, J. Satge, Main Group Met. Chem. 1987, 10, 69-76.

[126] J. Janssen, J. Magull, H. W. Roesky, Angew. Chem. 2002, 114, 1425-1427; Angew. Chem. Int. Ed. 2002, 41, 1365-1367.

[127] K. Wraage, L. Lameyer, D. Stalke, H. W. Roesky, Angew. Chem. 1999, 111, 542-544; Angew. Chem. Int. Ed. 1999, 38, 522-524.

[128] C. Ackerhans, H. W. Roesky, T. Labahn, J. Magull, Organometallics 2002, 21, 3671-3674.

[129] C. Ackerhans, Dissertation, Göttingen 2002.

[130] G. Bai, Y. Peng, H. W. Roesky, J. Li, H.-G. Schmidt, M. Noltemeyer, Angew. Chem. 2003, 115, 1164-1167; Angew. Chem. Int. Ed. 2003, 42, 1132-1135.

[131] G. Bai, H. W. Roesky, P. Lobinger, M. Noltemeyer, H.-G. Schmidt, Angew. Chem. 2001, 113, 2214-2217; Angew. Chem. Int. Ed. 2001, 40, 2156-2159.

[132] C. Elschenbroich, A. Salzer, Organometallchemie, 3. Aufl. Teubner, Stuttgart, 1993.

[133] J. Weidlein, U. Müller, K. Dehnicke, Schwingungsfrequenzen Bd. I, Thieme, Stuttgart, New York, 1981.

[134] R. J. Wehmschulte, P. P. Power, Inorg. Chem. 1994, 33, 5611-5612.

[135] C. Cui, H. W. Roesky, H. Hao, H.-G. Schmidt, M. Noltemeyer, Angew. Chem. 2000, 112, 1885-1887; Angew. Chem. Int. Ed. 2000, 39, 1815-1817.

[136] J. D. Gorden, A. Voigt, C. L. B. Macdonald, J. S. Silverman, A. H. Cowley, J. Am. Chem. Soc. 2000, 122, 950-951.

[137] M. R. Churchill, A. H. Reis, J. Chem. Soc., Dalton Trans. 1972, 1317-1320.

[138] H. Berwe, A. Haas, Chem. Ber. 1987, 120, 1175-1182.

[139] Autorenkollektiv, Organikum, 19. Aufl., Deutscher Verlag der Wissenschaften, Berlin, 1993.

[140] G. M. Sheldrick, Acta Crystallogr. 1990, A46, 467-473.

[141] G. M. Sheldrick, SHELX-97, Universität Göttingen, 1997.

[142] E. Krause, Ber. Dtsch. Chem. Ges. 1918, 51, 1447-1451.

[143] R. Bolton, J. P. B. Sandall, J. Chem. Soc. Perkin 2 1977, 278-280.

[144] M. Stender, R. J. Wright, B. E. Eichler, J. Prust, M. M. Olmstead, H. W. 
Roesky, P. P. Power, J. Chem. Soc., Dalton Trans. 2001, 3465-3469.

[145] B. Hübler-Blank, M. Witt, H. W. Roesky, J. Chem. Educ. 1993, 70, 408.

[146] H. W. Roesky, Inorg. Chem. 2001, 40, 6855-6856. 


\section{Lebenslauf}

Holger Hohmeister

geboren am 16.11.1972 in Eschwege

Eltern

Christa Hohmeister, geb. Englisch

Wilfried Hohmeister

Staatsangehörigkeit

deutsch

Familienstand

verheiratet

\section{Schulausbildung}

$1979-1983$

Mittelpunktschule Waldkappel

$1983-1989$

Friedrich-Wilhelm-Schule Eschwege

$1989-1992$

Oberstufengymnasium Eschwege

Mai 1992

Abitur

\section{Zivildienst}

August 1992 - Dezember 1992

Schule für Praktisch-Bildbare Oberhone

\section{Hochschulausbildung}

Mai 1993 - Mai 1998

Studium der Chemie an der Georg-AugustUniversität Göttingen

Juni 1995

Diplomvorprüfung

April 1997 - Januar 1998

Diplomarbeit im Arbeitskreis von Prof. Dr. Dr. h. c. mult. Roesky am Institut für Anorganische Chemie der Universität Göttingen

Mai 1998

Diplomhauptprüfung

Juni 1998 - März 2003

Anfertigung der vorliegenden Dissertation unter Anleitung von Prof. Dr. Dr. h. c. mult. Roesky am Institut für Anorganische Chemie der Universität Göttingen 